\title{
Gold-catalyzed Amide/Carbamate-linked N,O-Acetal Formation with Bulky Amides and Alcohols
}

Kosuke Ohsawa, Shota Ochiai, Junya Kubota, and Takayuki Doi *

Graduate School of Pharmaceutical Sciences, Tohoku University, 6-3 Aza-aoba, Aramaki, Aoba-ku, Sendai, 980-8578, Japan

*Corresponding author

E-mail address: doi_taka@mail.pharm.tohoku.ac.jp

Phone number: +81-22-795-6865

Fax number +81-22-795-6864

Table of Contents.

1. ${ }^{1} \mathrm{H}$ and ${ }^{13} \mathrm{C}\left\{{ }^{1} \mathrm{H}\right\}$ NMR spectra of synthetic compounds 


$$
\begin{aligned}
& \text { Allochn } \chi_{\text {coOEt }} \\
& 1 a \\
& { }^{1} \mathrm{H} \text { NMR }
\end{aligned}
$$

${ }^{1} \mathrm{H}$ NMR spectrum of $\mathbf{1 a}$

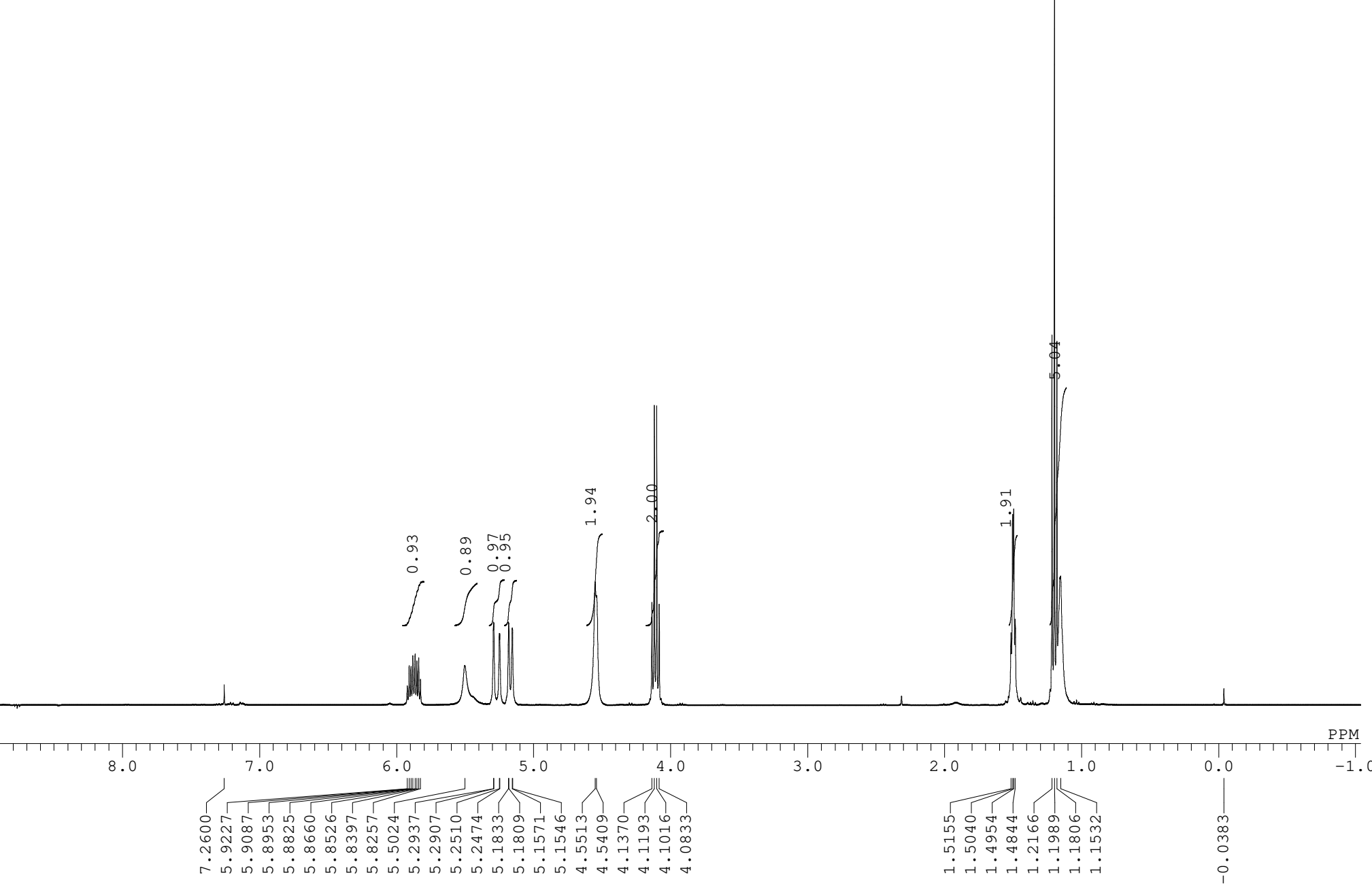




1a
$\begin{gathered}{ }^{13} \mathrm{C}\left\{{ }^{1} \mathrm{H}\right\} \mathrm{NMR} \\ \left(100 \mathrm{MHz} \text { in } \mathrm{CDCl}_{3}\right)\end{gathered}$

${ }^{13} \mathrm{C}\left\{{ }^{1} \mathrm{H}\right\}$ NMR spectrum of $\mathbf{1 a}$

$100 \mathrm{MHz}$ in $\mathrm{CDCl}_{3}$ )

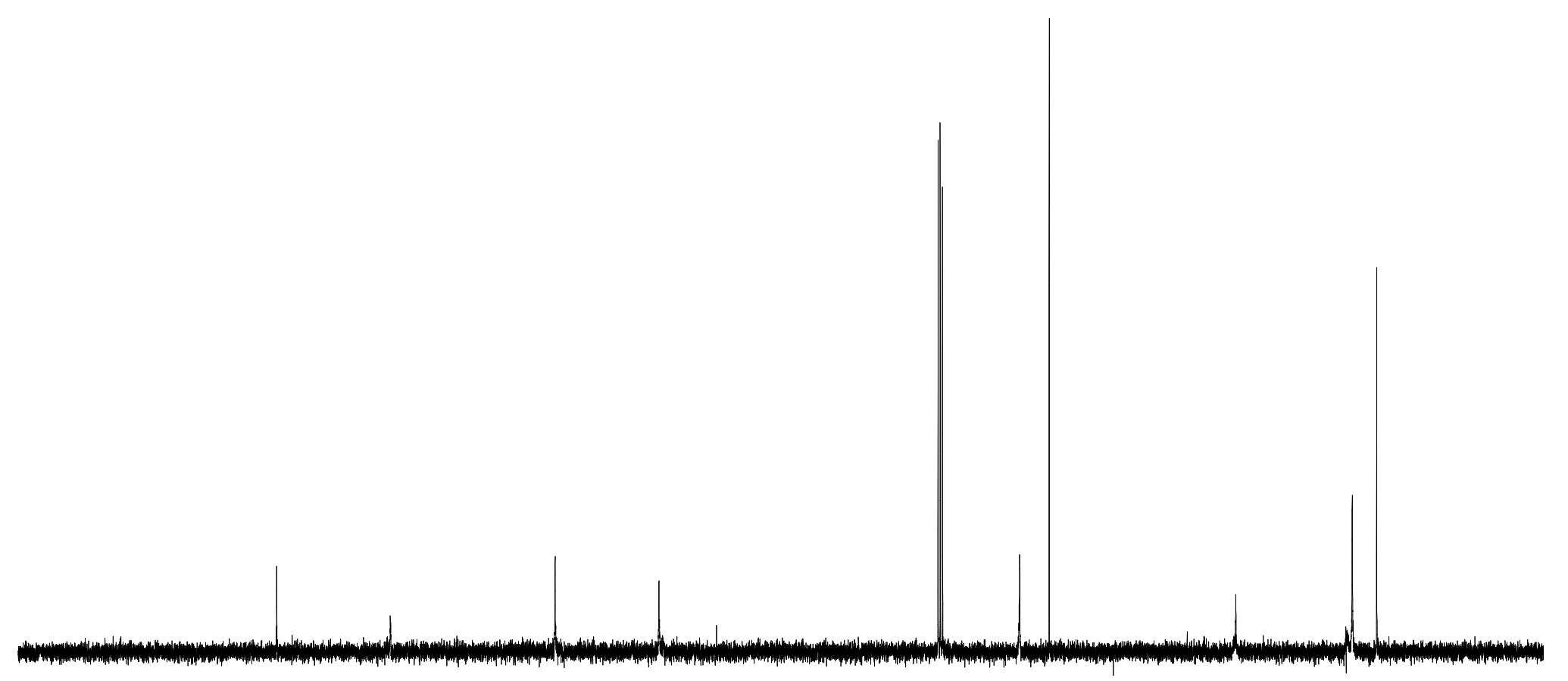

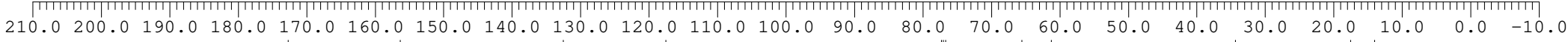

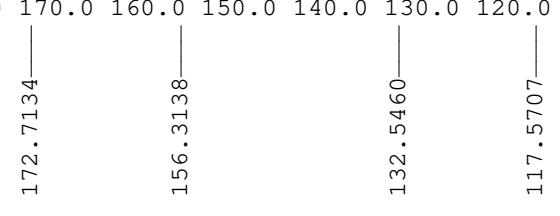

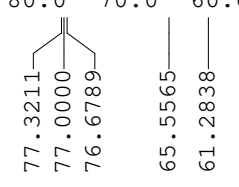

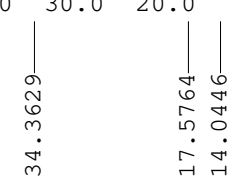




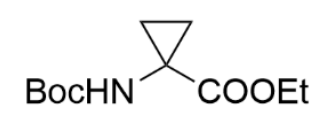

1b

${ }^{1} \mathrm{H}$ NMR

(600 MHz in $\mathrm{CDCl}_{3}$ )
${ }^{1} \mathrm{H}$ NMR spectrum of $\mathbf{1 b}$

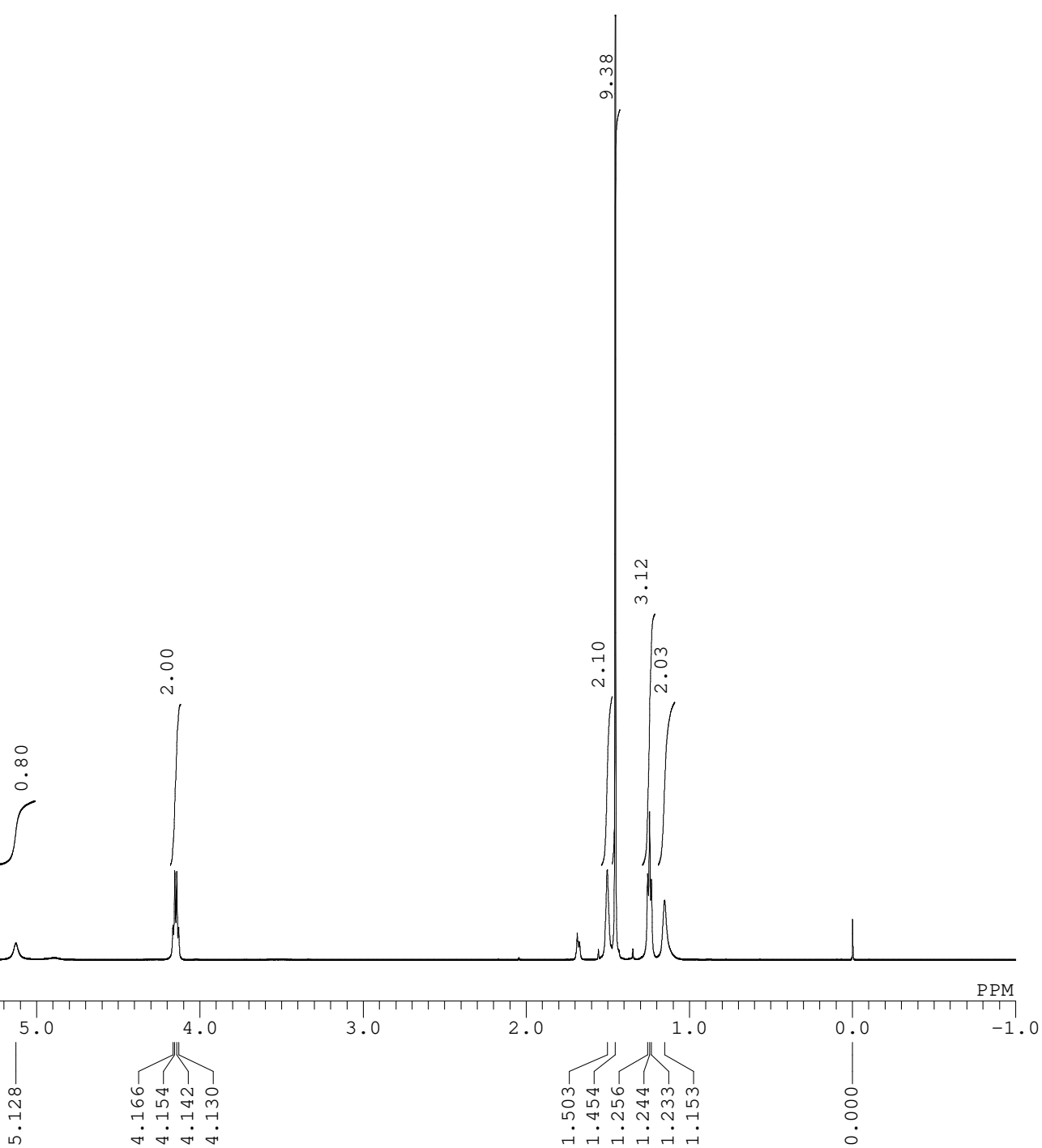

S4 


$$
\begin{gathered}
\text { BocHN }{ }_{\text {1b }}^{\left.{ }_{\text {COOEt }}^{13} \mathrm{C}_{\{1}^{1} \mathrm{H}\right\} \mathrm{NMR}^{\mathrm{NM}}} \\
\left(150 \mathrm{MHz} \text { in } \mathrm{CDCl}_{3}\right)
\end{gathered}
$$

${ }^{13} \mathrm{C}\left\{{ }^{1} \mathrm{H}\right\}$ NMR spectrum of $\mathbf{1 b}$

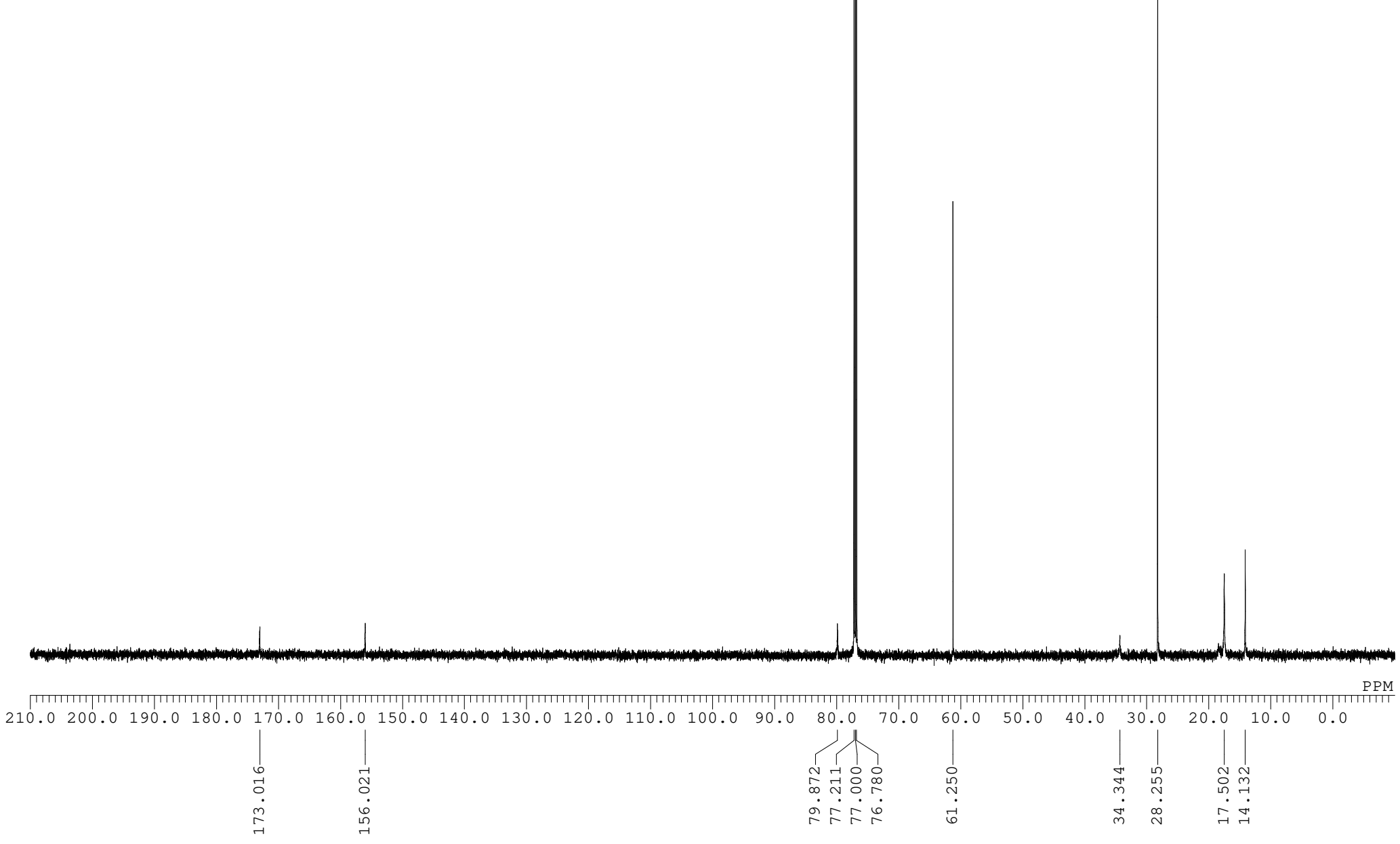




$$
\begin{aligned}
& { }_{\mathrm{H}_{2} \mathrm{~N}} \chi_{\mathrm{COOEt}}^{\cdot \mathrm{HCl}} \\
& { }^{1} \mathrm{H} \text { NMR }
\end{aligned}
$$

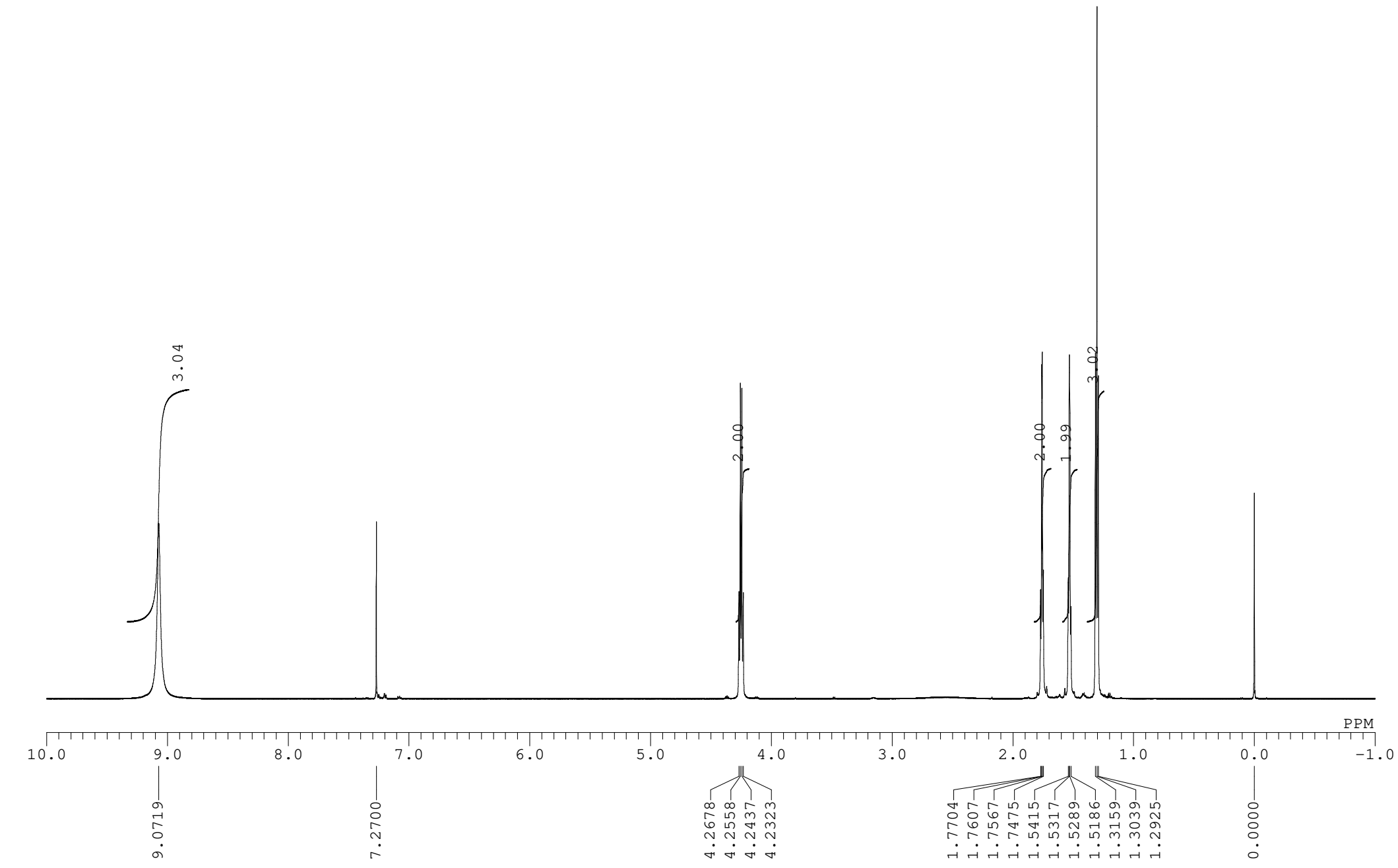




$$
\begin{gathered}
\left.{ }_{\mathrm{H}_{2} \mathrm{~N}}{ }_{\mathrm{COOEt}} \cdot{ }^{1} \mathrm{HCl}\right\} \mathrm{NMR} \\
\left(150 \mathrm{MHz} \text { in } \mathrm{CDCl}_{3}\right)
\end{gathered}
$$

(150 $\mathrm{MHz}_{\mathrm{in}} \mathrm{CDCl}_{3}$ )

${ }^{13} \mathrm{C}\left\{{ }^{1} \mathrm{H}\right\}$ NMR spectrum of ethyl 1-aminocyclopropane-1-carboxylate hydrochloride

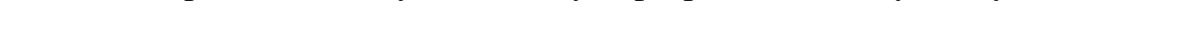




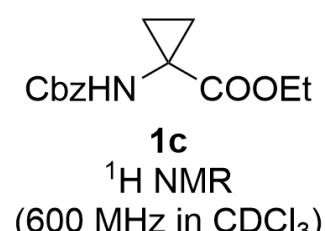

${ }^{1} \mathrm{H}$ NMR spectrum of $\mathbf{1 c}$

(600 MHz in $\mathrm{CDCl}_{3}$ )

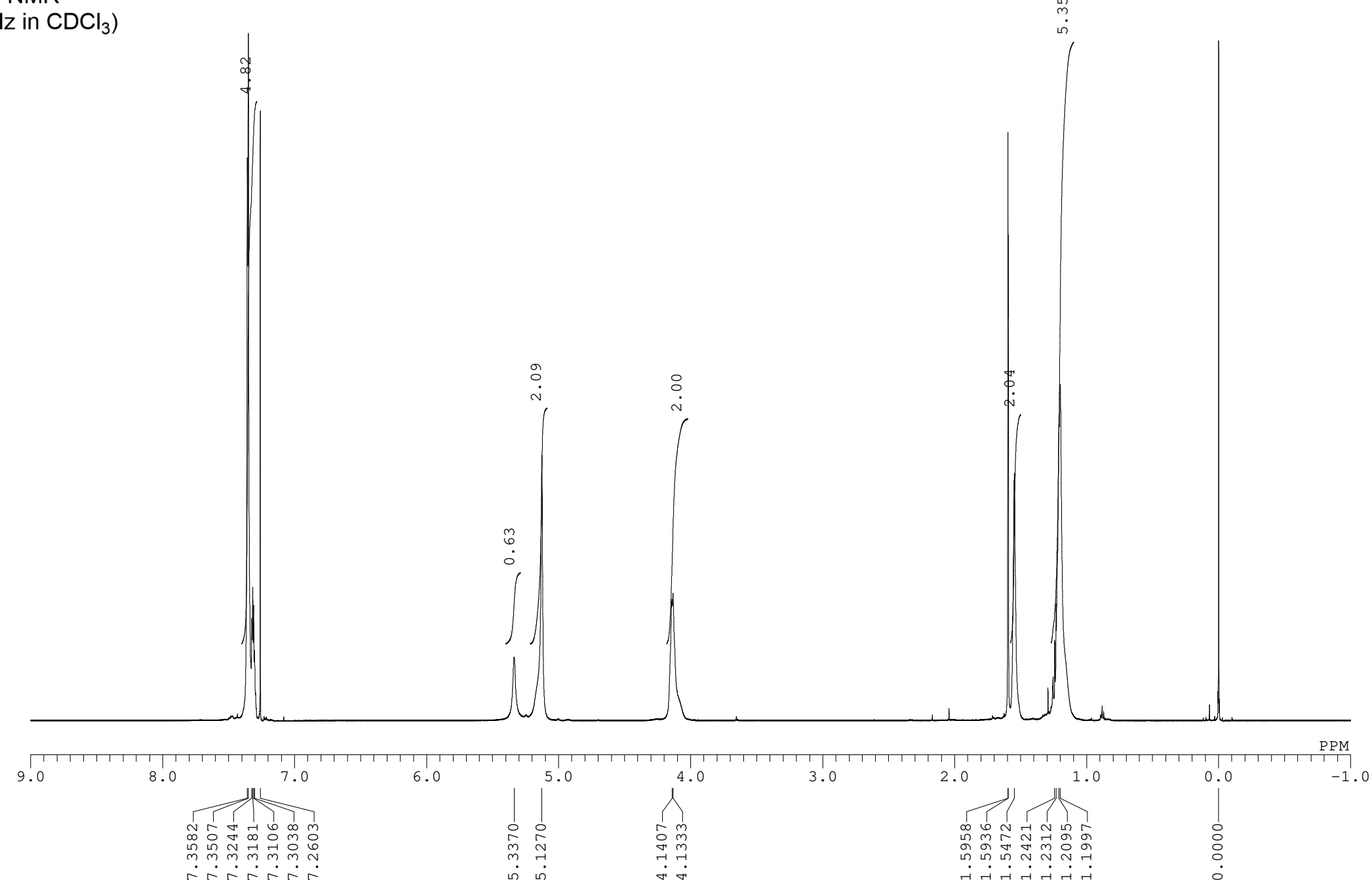




$$
\begin{aligned}
& { }_{\text {cbzHN }} \gamma_{\text {coOEt }} \\
& \text { 1c }
\end{aligned}
$$

${ }^{13} \mathrm{C}\left\{{ }^{1} \mathrm{H}\right\}$ NMR spectrum of $\mathbf{1 c}$

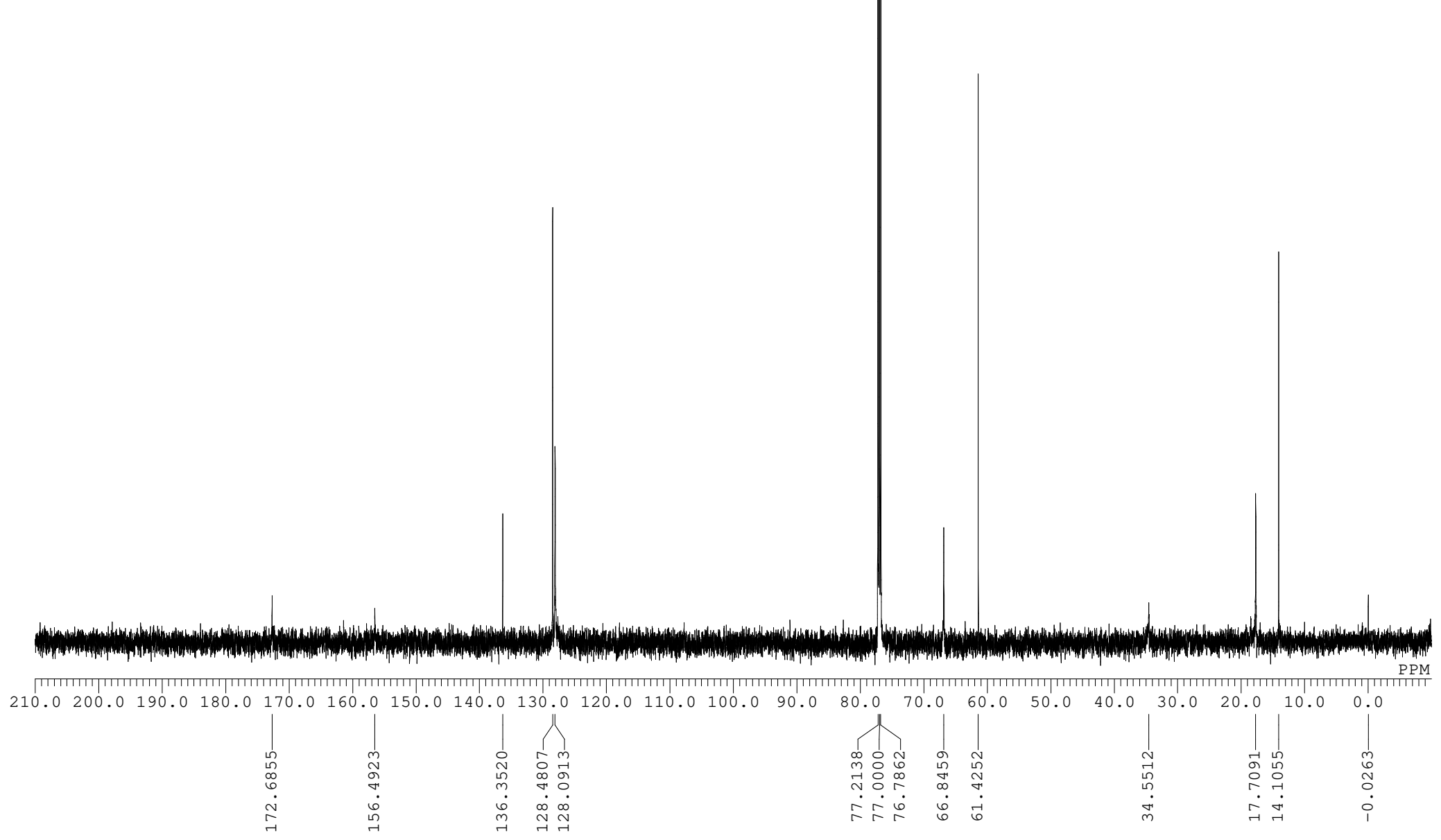


$\underset{\substack{\text { 1d } \\{ }_{\text {FmocHN } \mathrm{NMR}}}}{\left(600 \mathrm{MHz} \text { in } \mathrm{CDCl}_{3}\right)}$

${ }^{1} \mathrm{H}$ NMR spectrum of $\mathbf{1 d}$

3:1 rotamer mixture

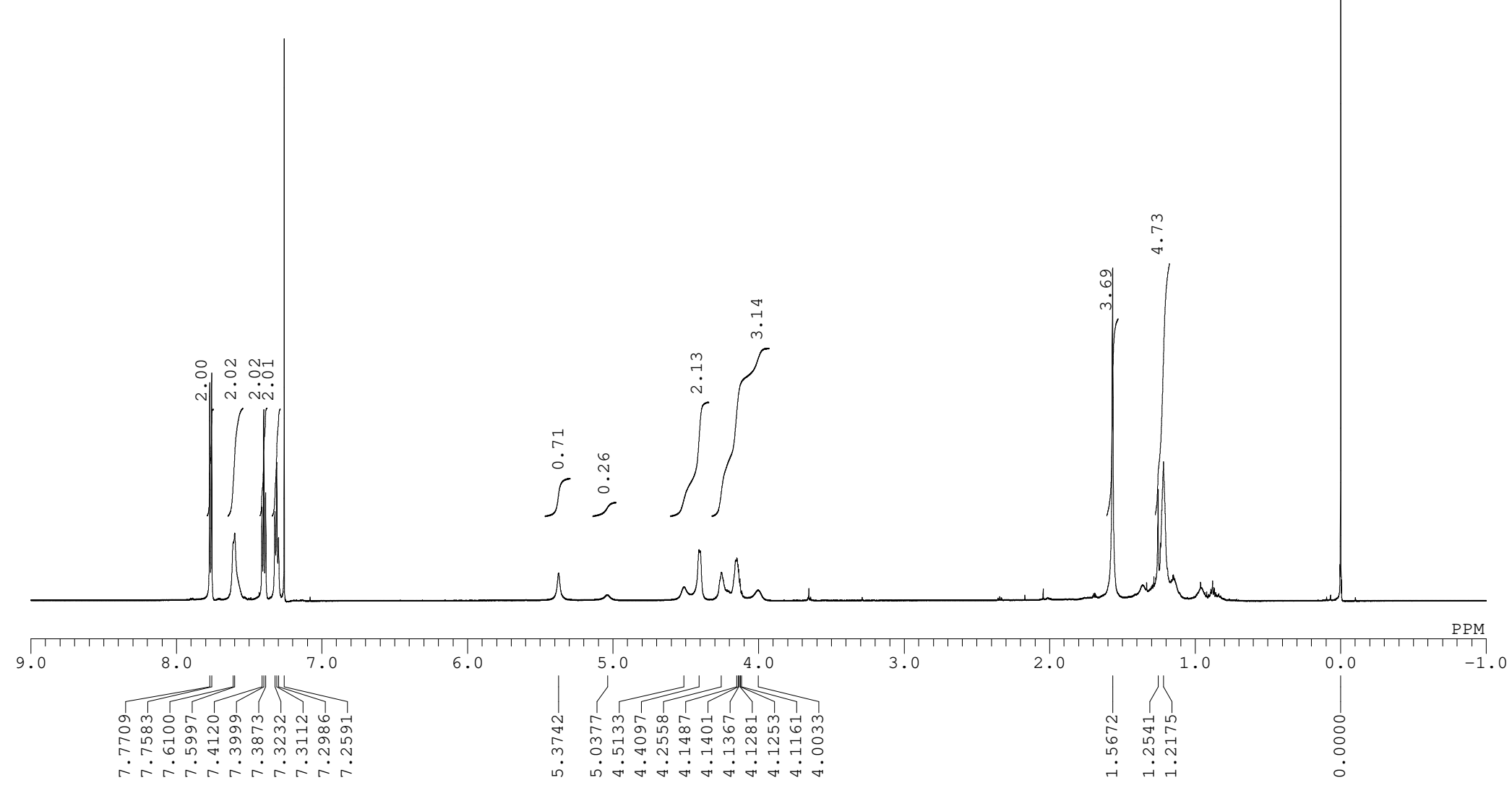


${ }_{\text {FmochN }} \chi_{\text {cooEt }}$

${ }^{13} \mathrm{C}\left\{{ }^{1} \mathrm{H}\right\}$ NMR spectrum of $\mathbf{1 d}$

$\mathrm{C}\left\{{ }^{1} \mathrm{H}\right\}$ NMR

$\left(150 \mathrm{MHz}\right.$ in $\mathrm{CDCl}_{3}$ )

3:1 rotamer mixture

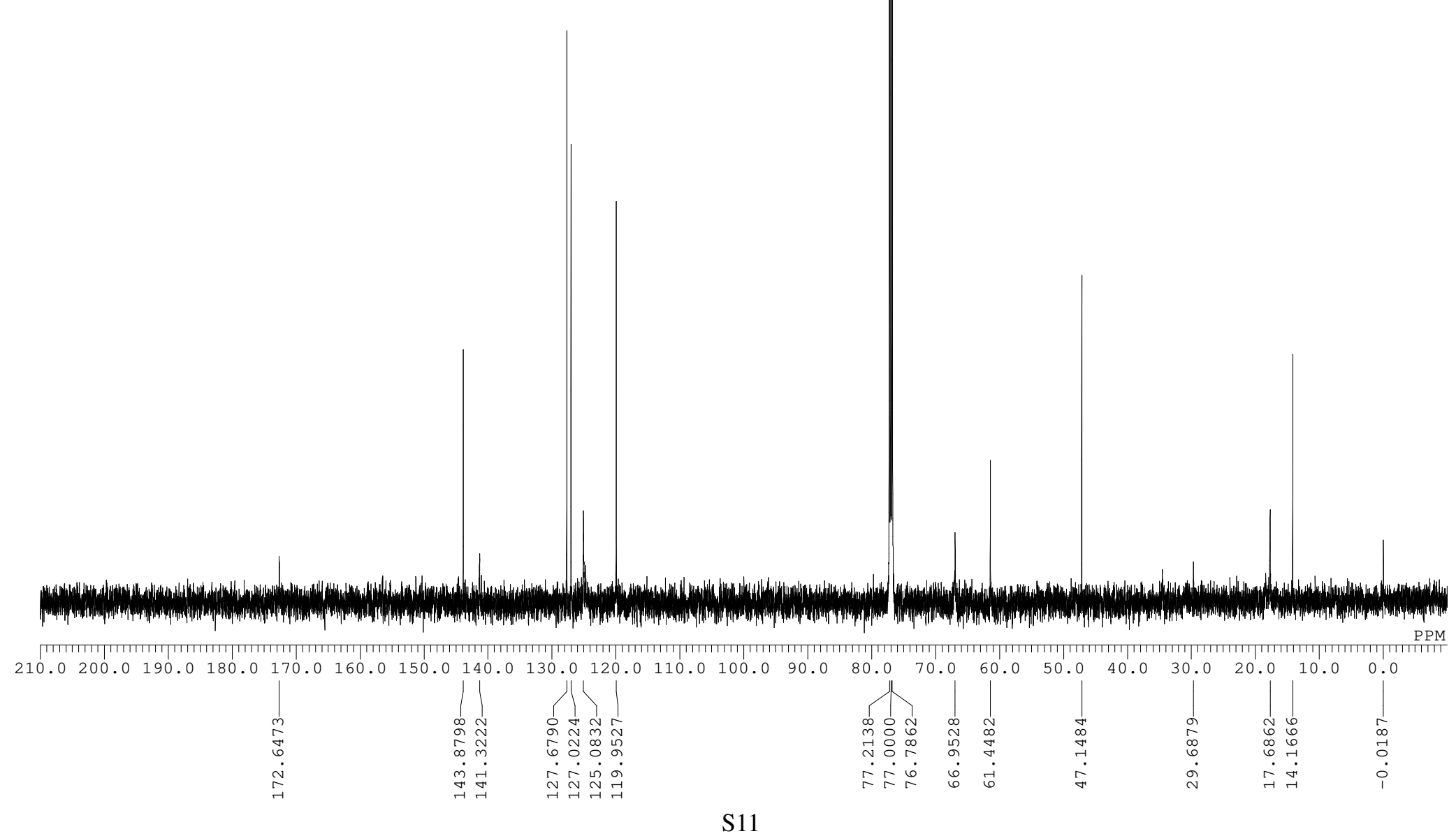




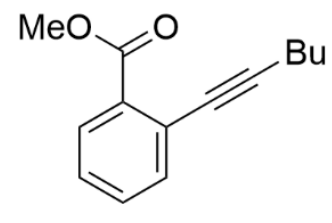

${ }^{1}$ H NMR spectrum of methyl 2-(hex-1-yn-1-yl)benzoate

$\left(400 \mathrm{MHz}^{\text {in } \mathrm{CDCl}_{3}}\right)$
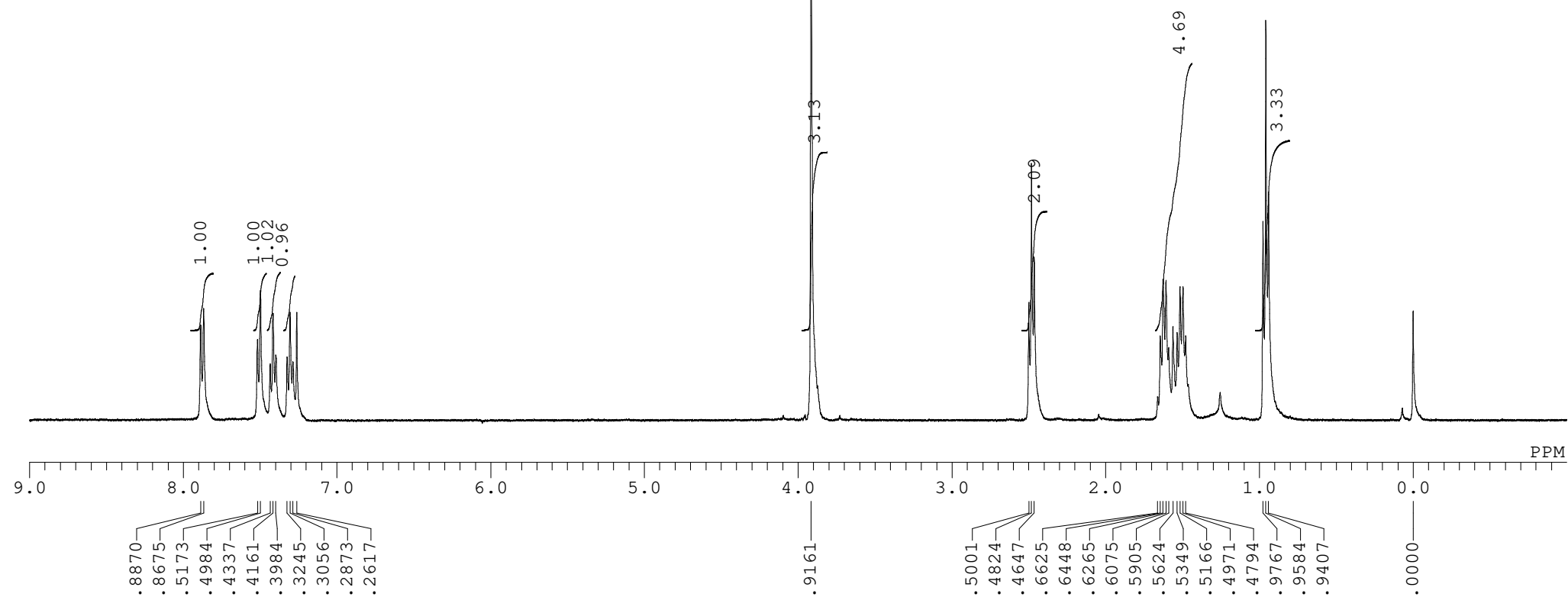

6.0

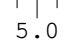

3.0

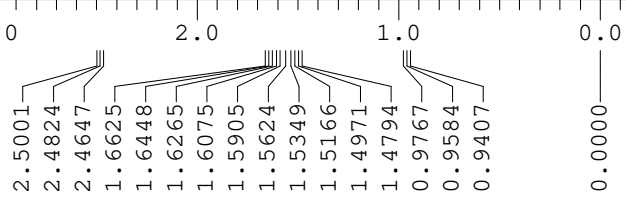


$\mathrm{MeO}$

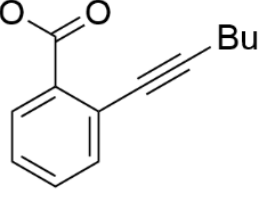

${ }^{13} \mathrm{C}\left\{{ }^{1} \mathrm{H}\right\} \mathrm{NMR}$

$\left(100 \mathrm{MHz}\right.$ in $\left.\mathrm{CDCl}_{3}\right)$
${ }^{13} \mathrm{C}\left\{{ }^{1} \mathrm{H}\right\}$ NMR spectrum of methyl 2-(hex-1-yn-1-yl)benzoate

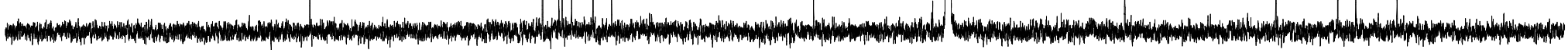

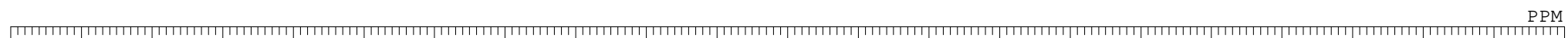

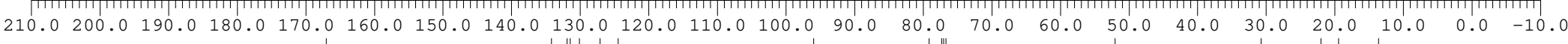

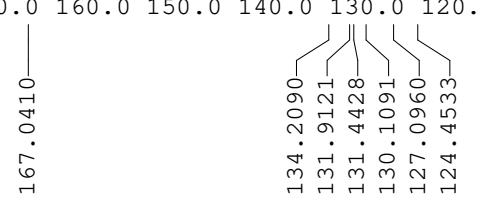

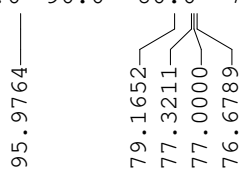

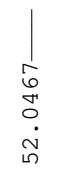

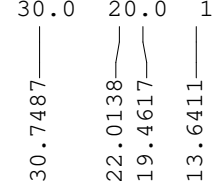




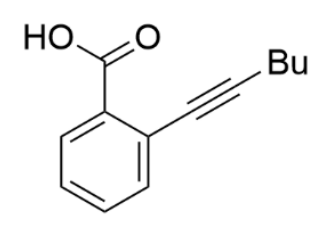

2

${ }^{1} \mathrm{H}$ NMR

$\left(400 \mathrm{MHz}\right.$ in $\mathrm{CDCl}_{3}$ )
${ }^{1} \mathrm{H}$ NMR spectrum of $\mathbf{2}$

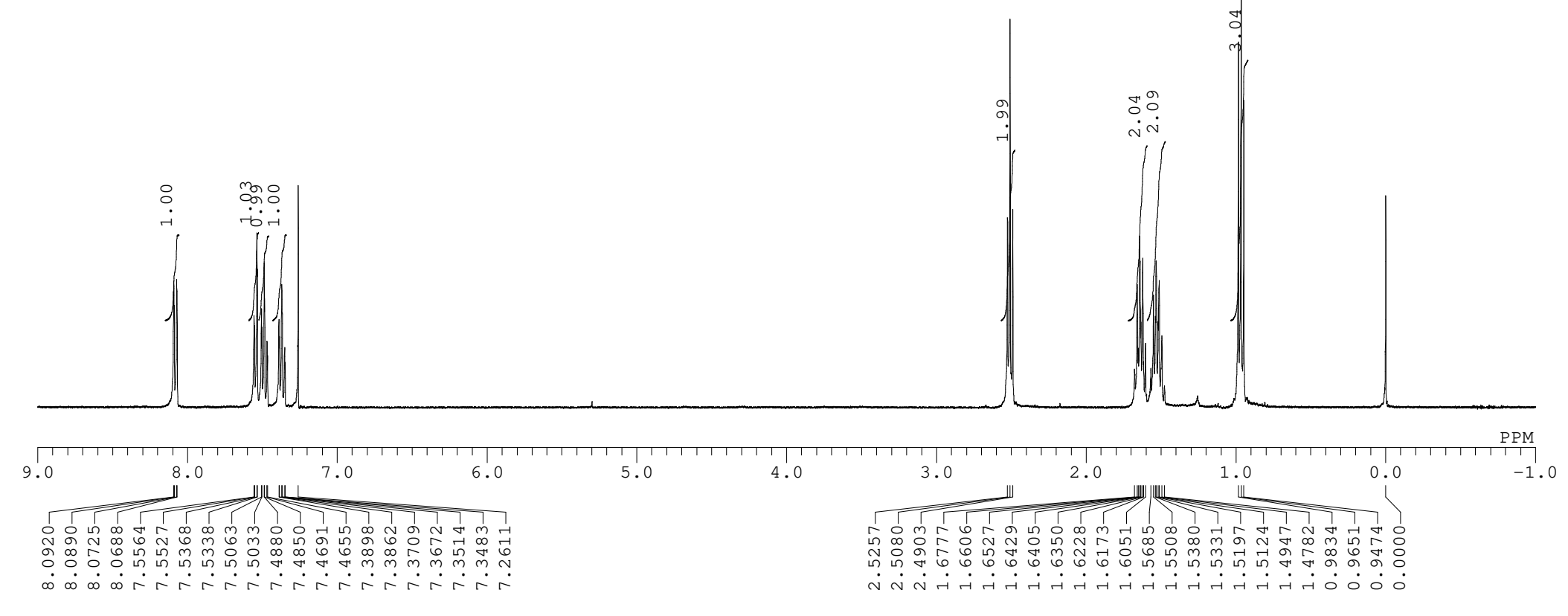


$\mathrm{HO}$

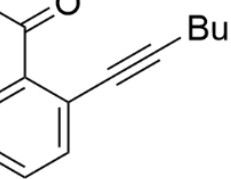

${ }^{13} \mathrm{C}\left\{{ }^{1} \mathrm{H}\right\} \mathrm{NMR}$

$\left(100 \mathrm{MHz}\right.$ in $\left.\mathrm{CDCl}_{3}\right)$
${ }^{13} \mathrm{C}\left\{{ }^{1} \mathrm{H}\right\}$ NMR spectrum of 2

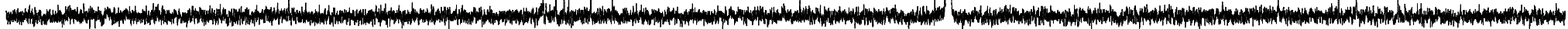

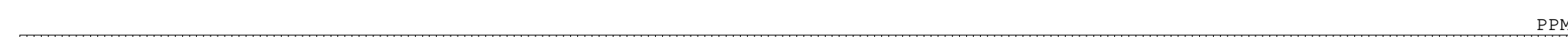

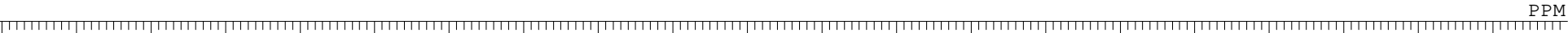

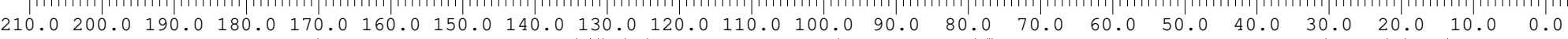

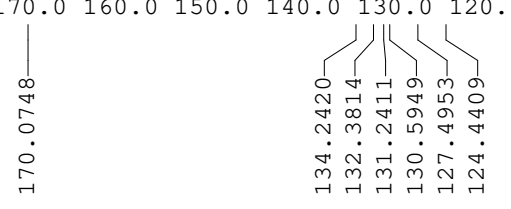




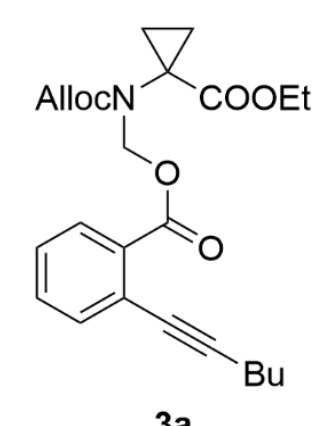

${ }^{1} \mathrm{H}$ NMR spectrum of $\mathbf{3 a}$

$3 a$

(400 $\mathrm{MHz}$ in $\mathrm{CDCl}_{3}$ )

rotamer mixture

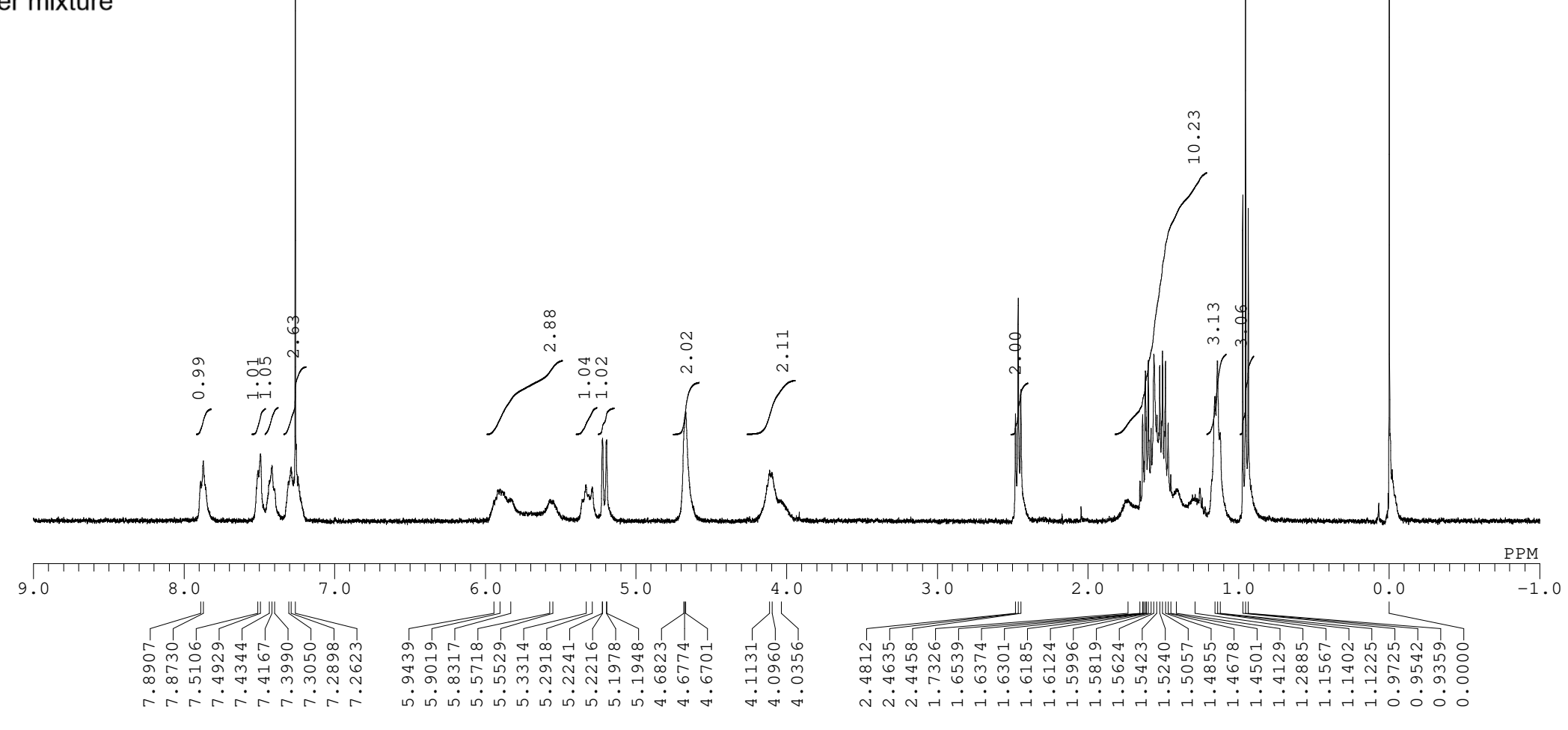




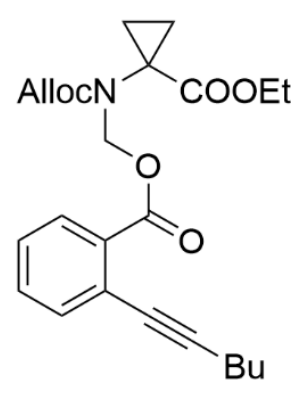

${ }^{13} \mathrm{C}\left\{{ }^{1} \mathrm{H}\right\}$ NMR spectrum of $\mathbf{3 a}$

$3 a$

${ }^{13} \mathrm{C}\left\{{ }^{1} \mathrm{H}\right\} \mathrm{NMR}$

$\left(100 \mathrm{MHz}\right.$ in $\left.\mathrm{CDCl}_{3}\right)$

rotamer mixture

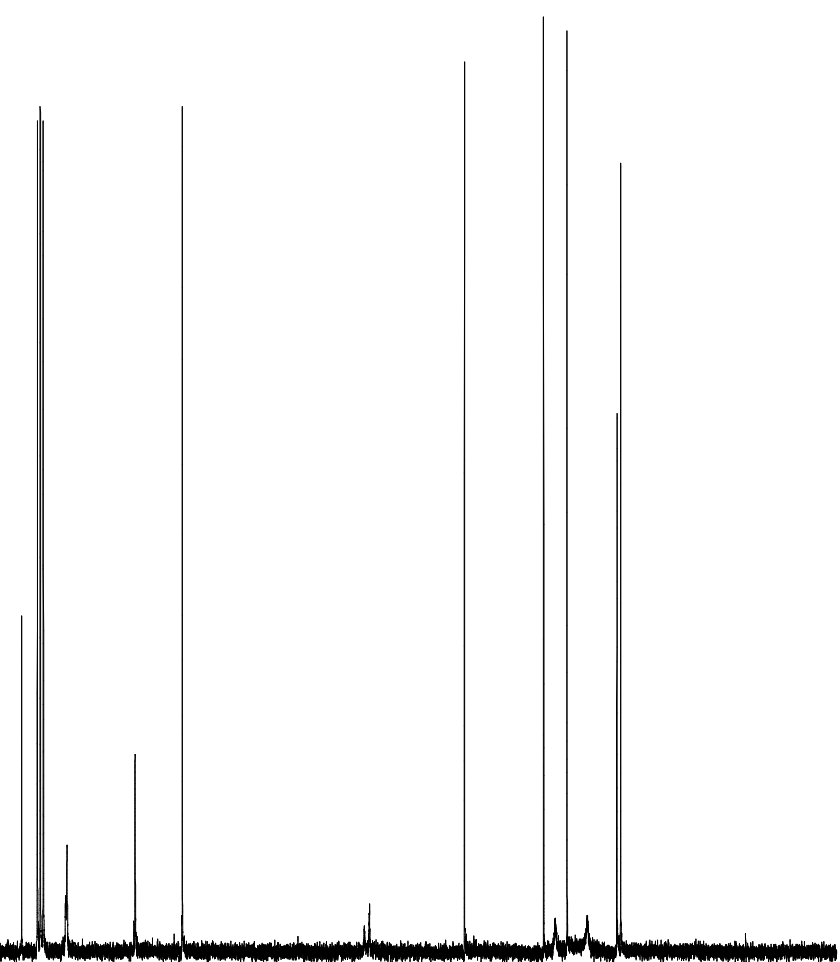

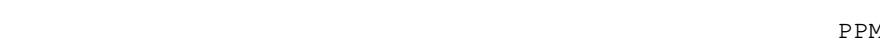

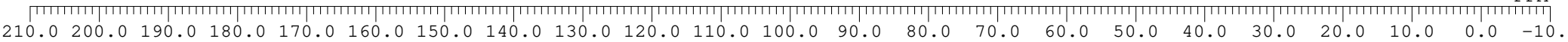

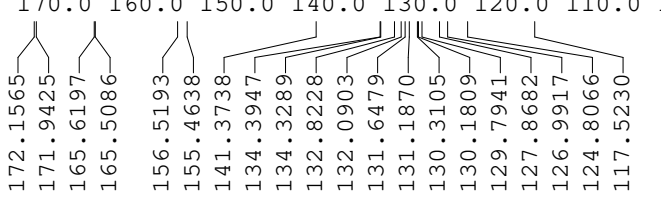

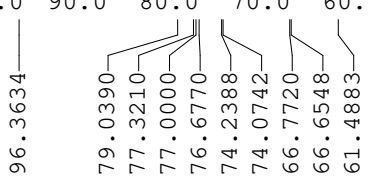

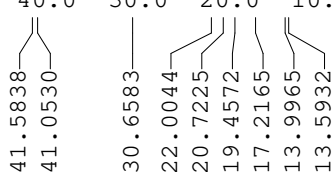




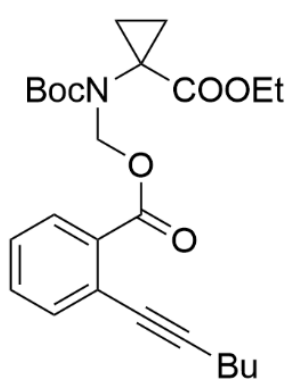

${ }^{1} \mathrm{H}$ NMR spectrum of $\mathbf{3 b}$

3b

${ }^{1} \mathrm{H}$ NMR

(600 MHz in $\mathrm{CDCl}_{3}$ )

rotamer mixture

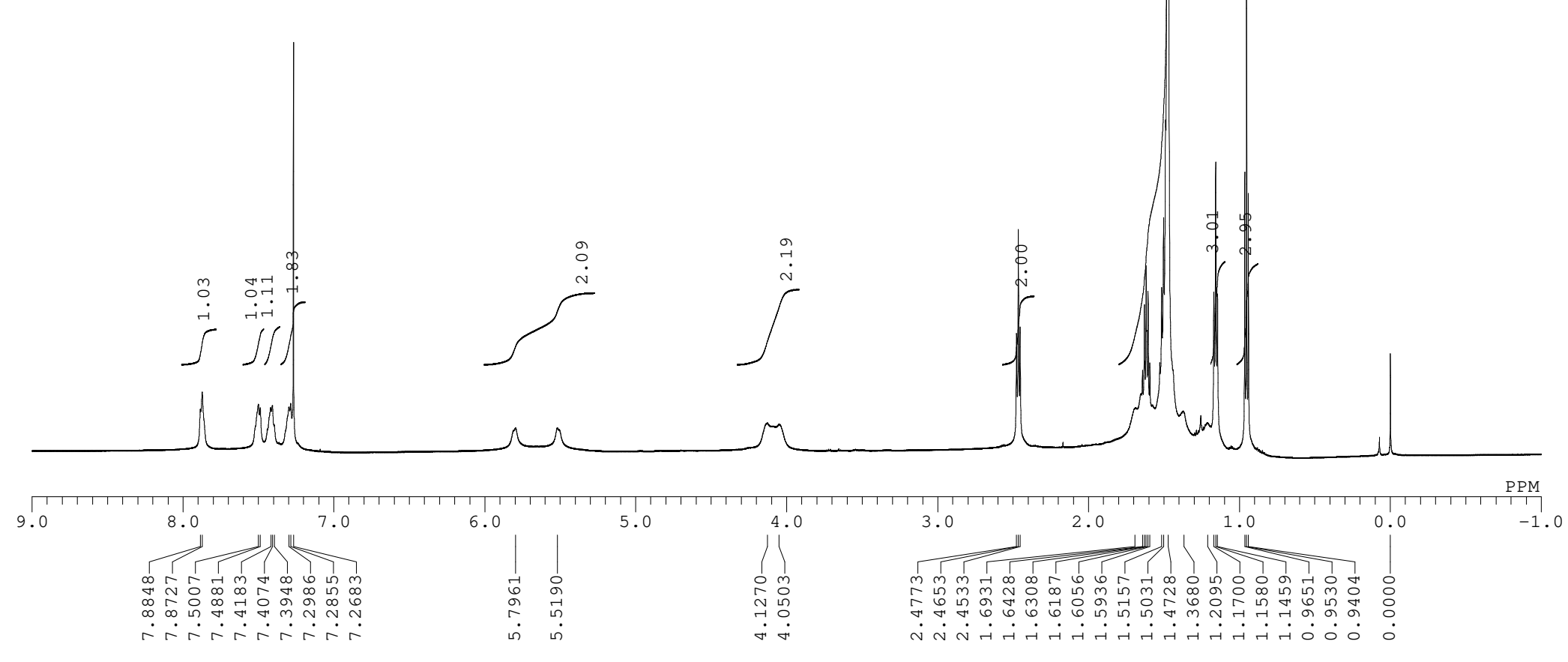




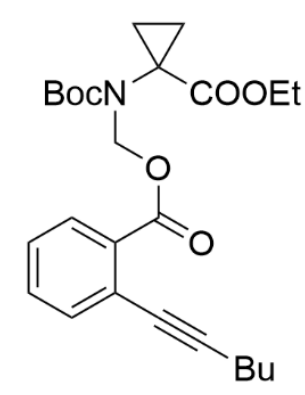

${ }^{13} \mathrm{C}\left\{{ }^{1} \mathrm{H}\right\}$ NMR spectrum of $\mathbf{3 b}$

${ }^{13} \mathrm{C}\left\{{ }^{1} \mathrm{H}\right\}$ NMR

(150 $\mathrm{MHz}$ in $\mathrm{CDCl}_{3}$ )

rotamer mixture 


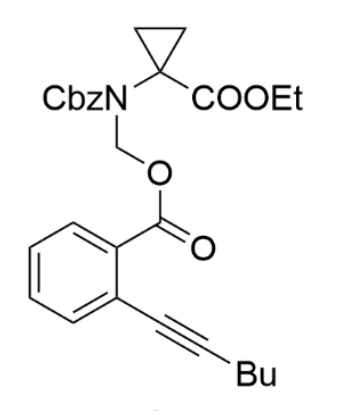

3c

${ }^{1} \mathrm{H}$ NMR (600 $\mathrm{MHz}$ in $\mathrm{CDCl}_{3}$ )

rotamer mixture
${ }^{1} \mathrm{H}$ NMR spectrum of $\mathbf{3 c}$

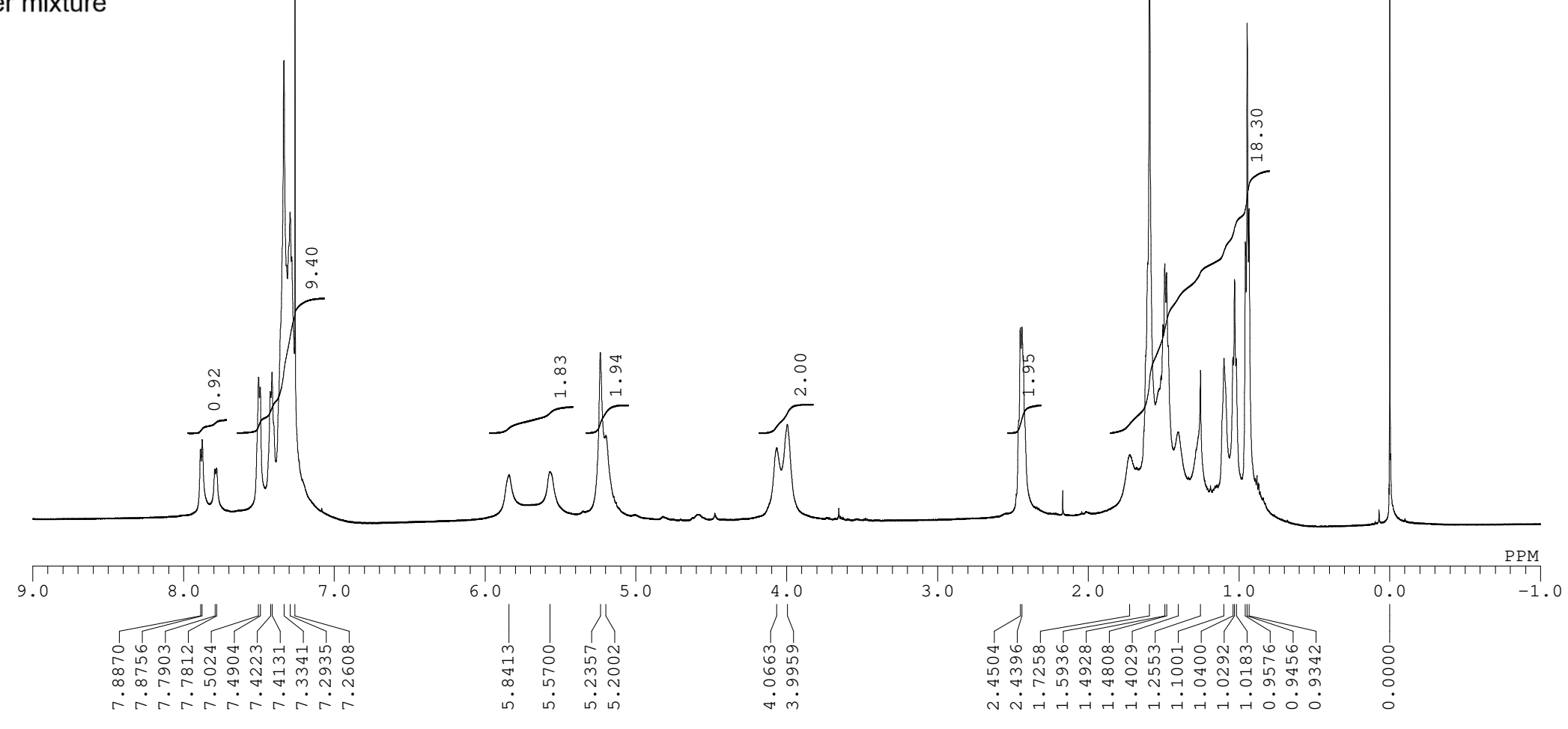




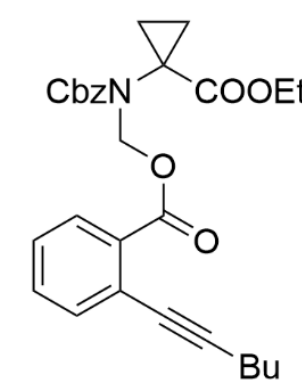

${ }^{13} \mathrm{C}\left\{{ }^{1} \mathrm{H}\right\}$ NMR spectrum of $\mathbf{3 c}$

$3 \mathrm{c}$

${ }^{13} \mathrm{C}\left\{{ }^{1} \mathrm{H}\right\}$ NMR (150 $\mathrm{MHz}$ in $\mathrm{CDCl}_{3}$ )

rotamer mixture

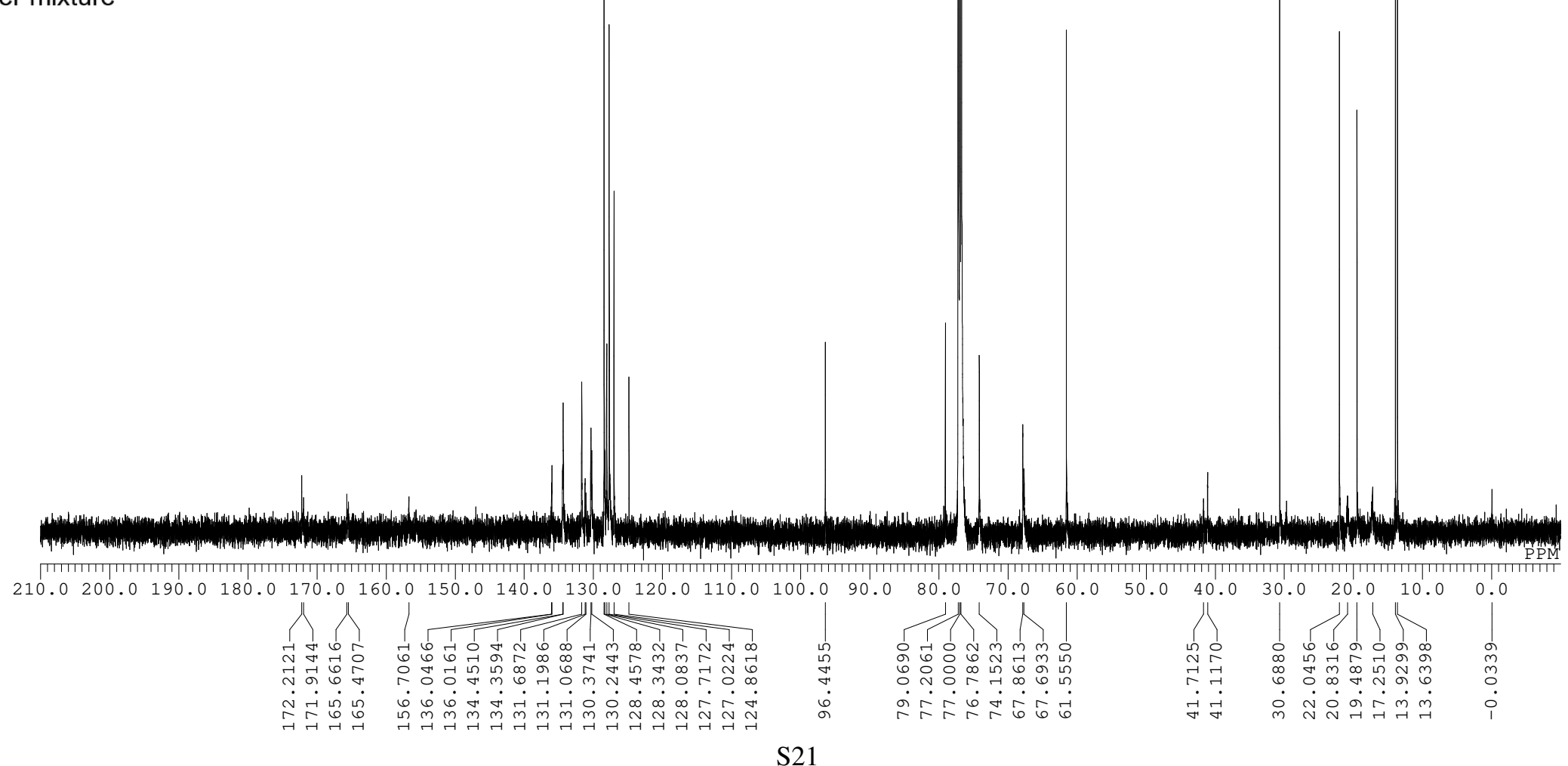




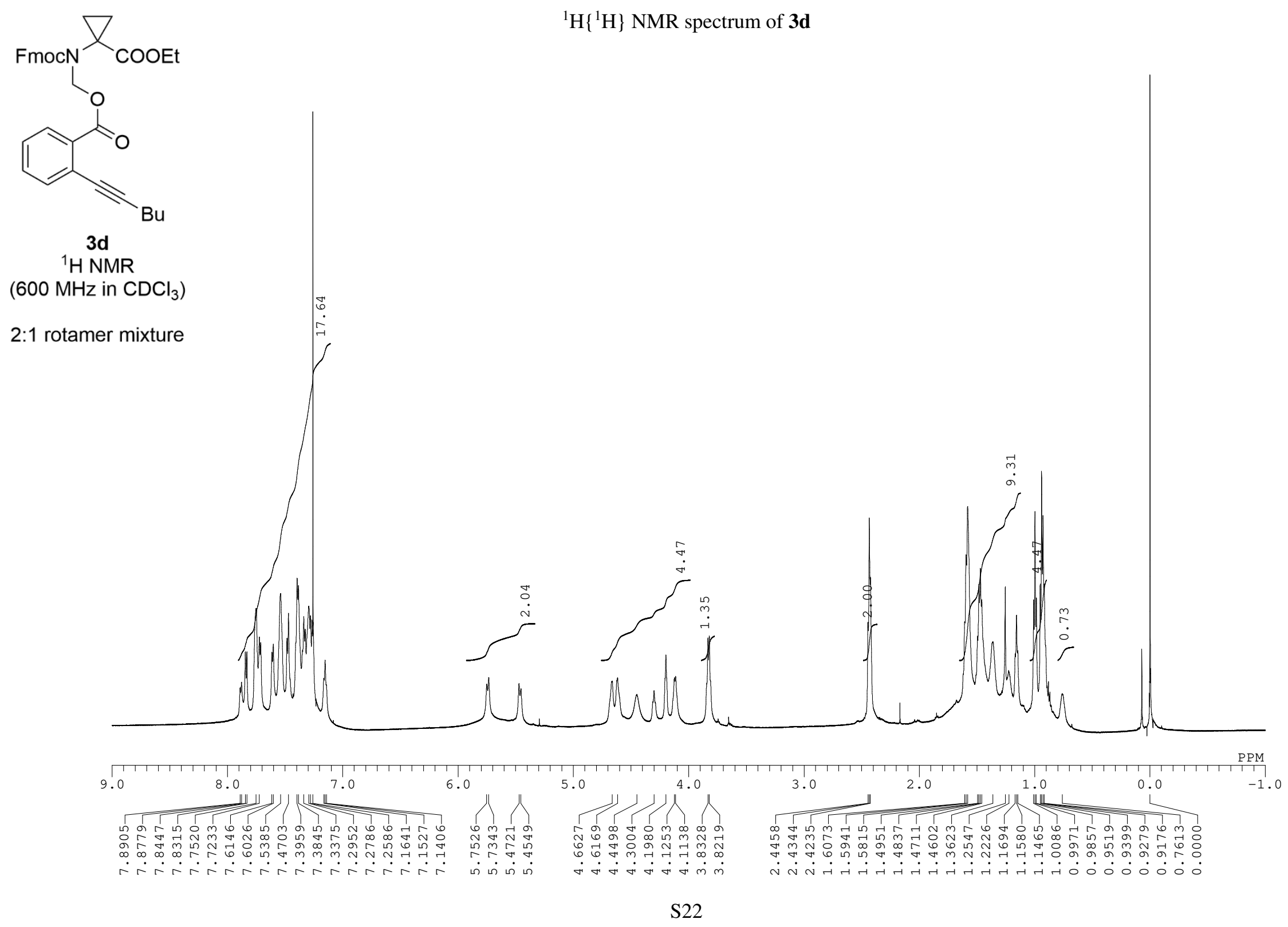




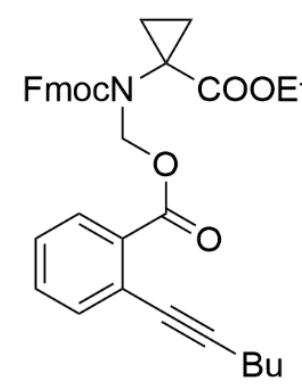

${ }^{13} \mathrm{C}\left\{{ }^{1} \mathrm{H}\right\}$ NMR spectrum of $\mathbf{3 d}$

3d

${ }^{13} \mathrm{C}\left\{{ }^{1} \mathrm{H}\right\}$ NMR

(150 $\mathrm{MHz}$ in $\mathrm{CDCl}_{3}$ )

2:1 rotamer mixture

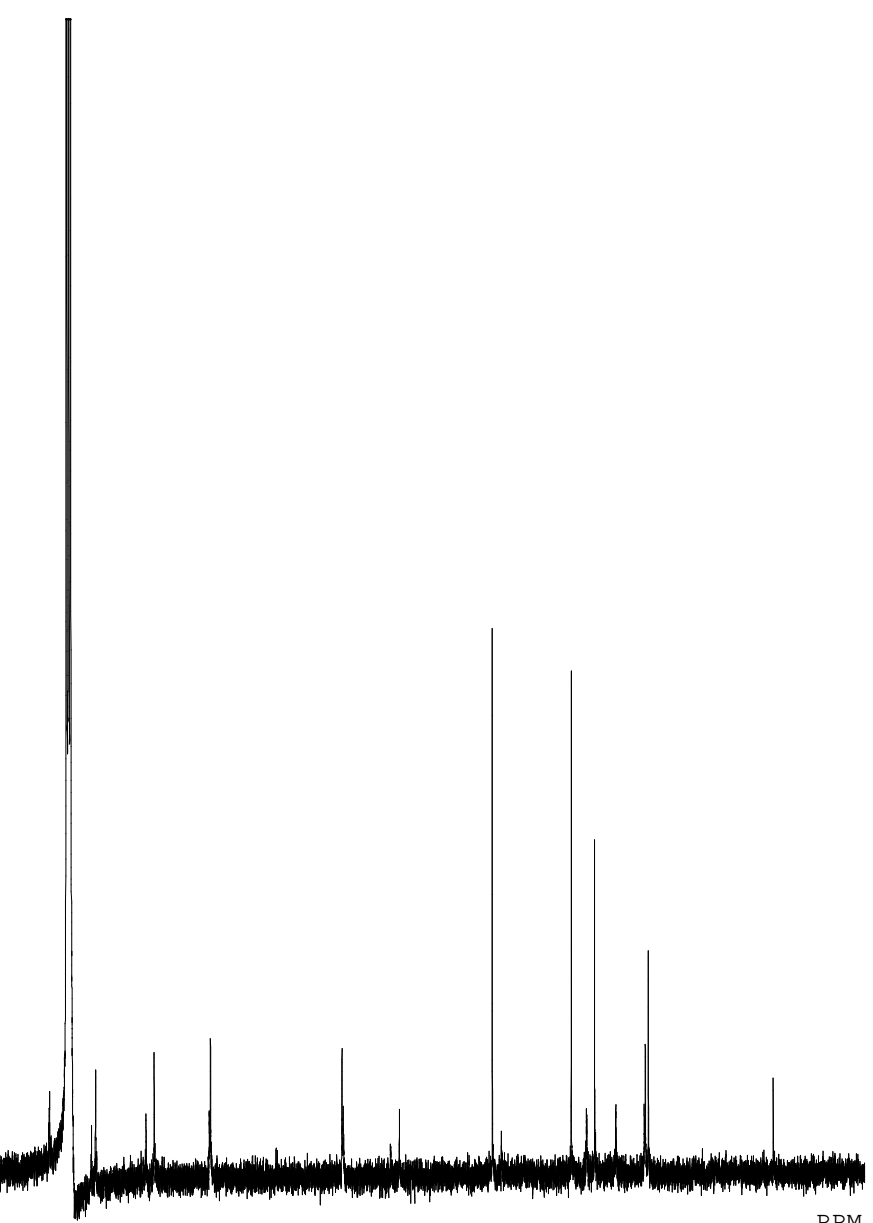

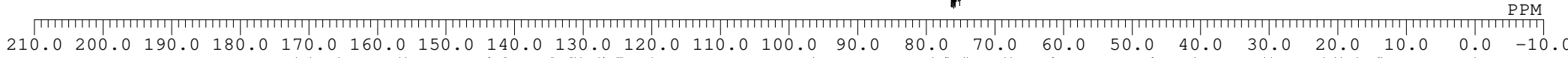

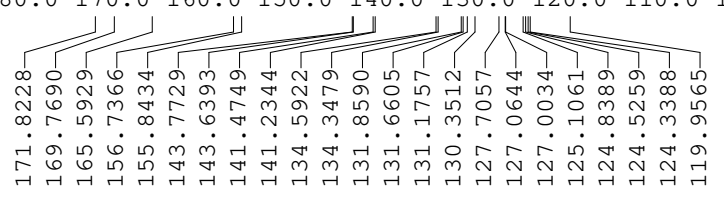

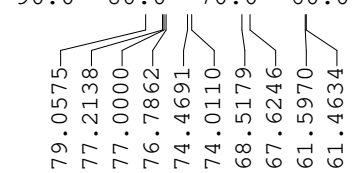

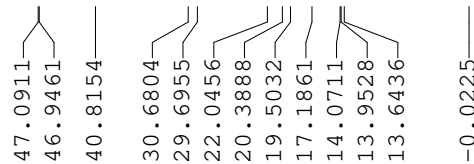




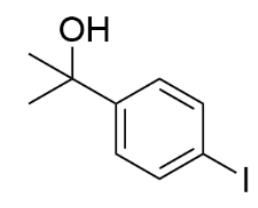

4d
${ }^{1} \mathrm{H} N \mathrm{NMR}$

(400 MHz in $\mathrm{CDCl}_{3}$ )
${ }^{1} \mathrm{H}$ NMR spectrum of $\mathbf{4 d}$

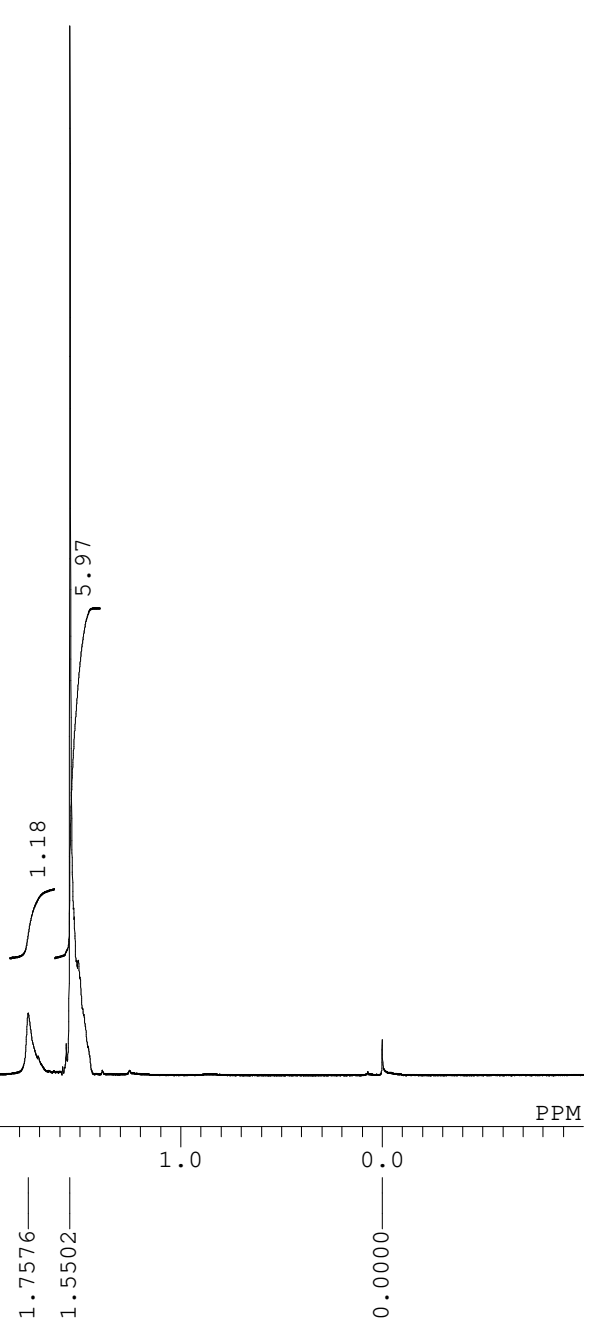

S24 


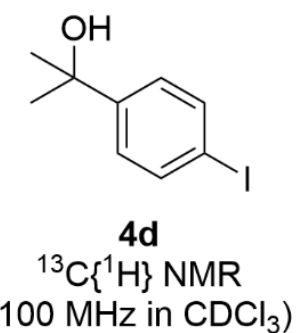

${ }^{13} \mathrm{C}\left\{{ }^{1} \mathrm{H}\right\}$ NMR spectrum of $\mathbf{4 d}$

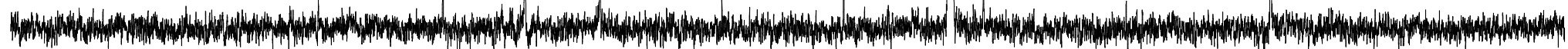

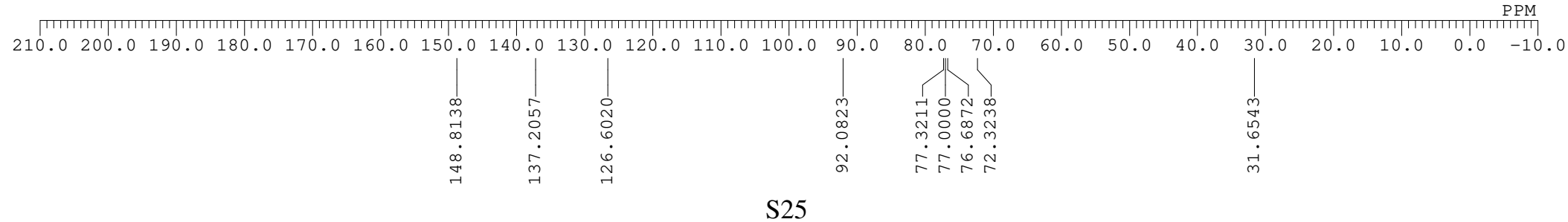




$$
{ }_{{ }^{1} \mathrm{HNMR}}^{\mathrm{HO} \mathrm{TSO}^{\prime}}
$$

$\left(400 \mathrm{MHz}\right.$ in $\left.\mathrm{CDCl}_{3}\right)$

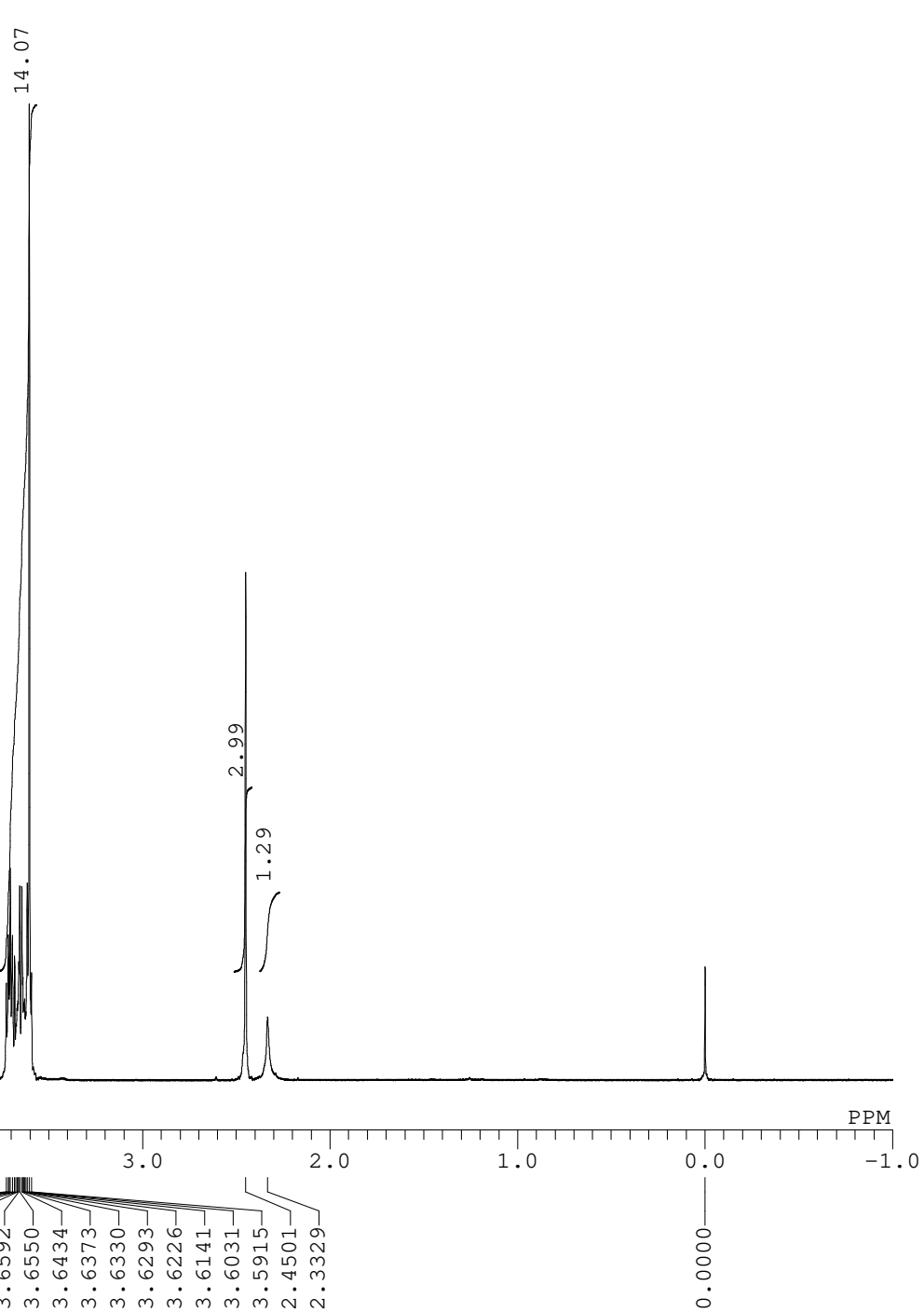




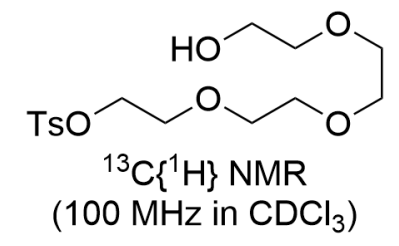

${ }^{13} \mathrm{C}\left\{{ }^{1} \mathrm{H}\right\}$ NMR spectrum of 14-hydroxy-3,6,9,12-tetraoxatetradecyl-4'-methylbenzolsulfonate ${ }^{13} \mathrm{C}\left\{{ }^{1} \mathrm{H}\right\} \mathrm{NMR}$
$\left(100 \mathrm{MHz}\right.$ in $\left.\mathrm{CDCl}_{3}\right)$

\section{'}




$$
\left(400 \mathrm{MHz} \text { in } \mathrm{CDCl}_{3}\right)
$$

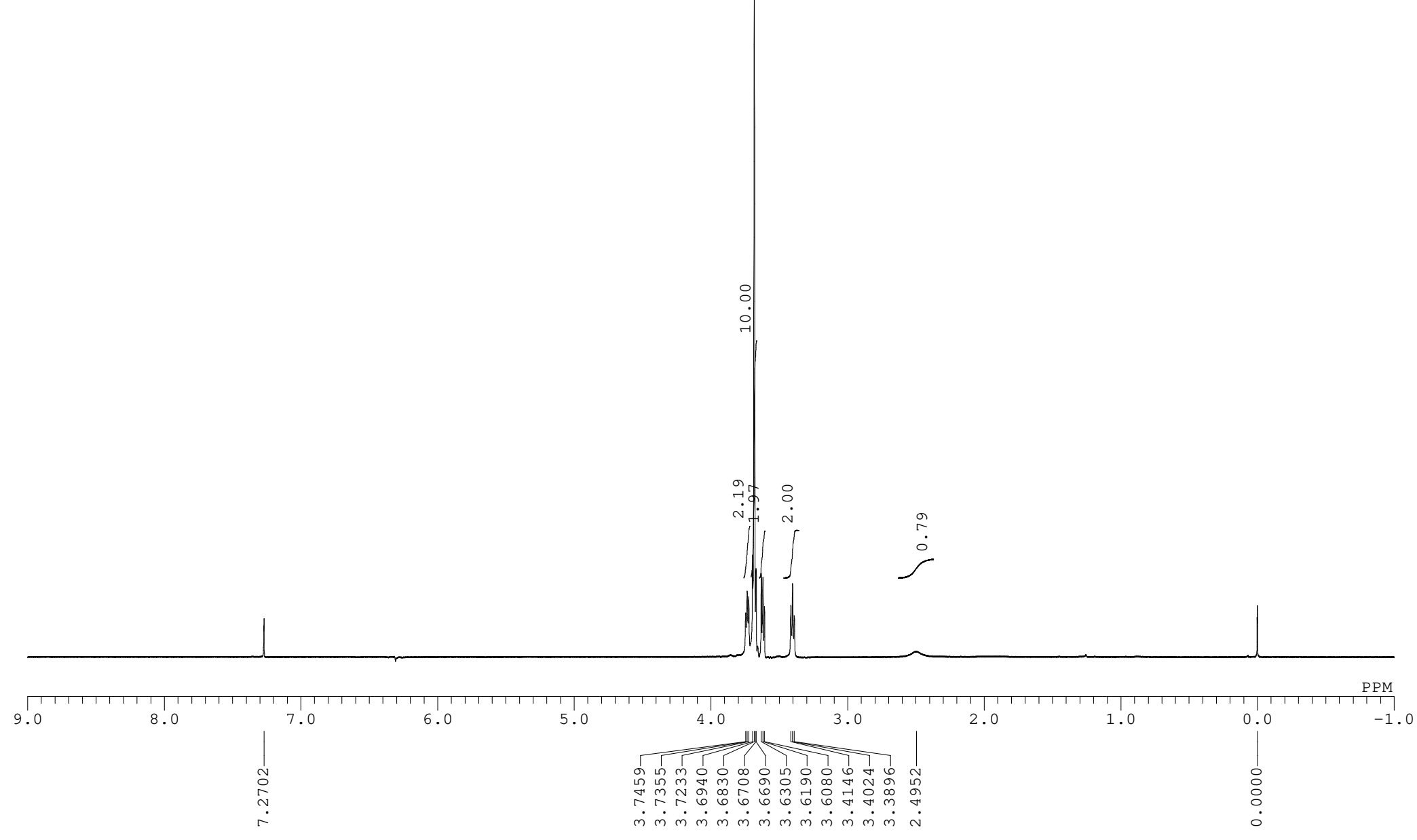




$$
\begin{aligned}
& \mathrm{N}^{\mathrm{O}} \overbrace{\mathrm{O}} \\
& 4 \text { e }
\end{aligned}
$$

${ }^{13} \mathrm{C}\left\{{ }^{1} \mathrm{H}\right\}$ NMR spectrum of $\mathbf{4 e}$

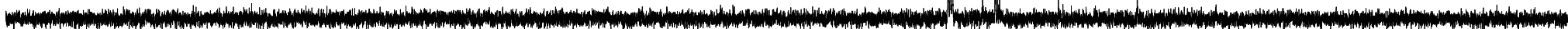

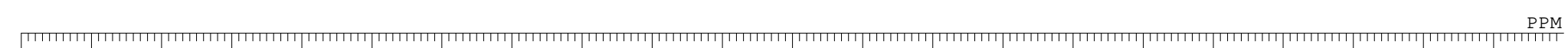

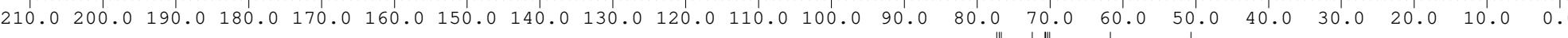

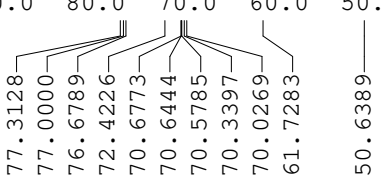




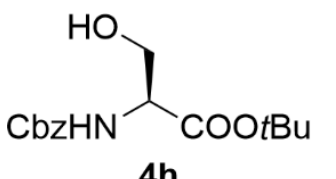

${ }^{1} \mathrm{H}$ NMR spectrum of $\mathbf{4 h}$

(400 $\mathrm{MHz}$ in $\mathrm{CDCl}_{3}$ )

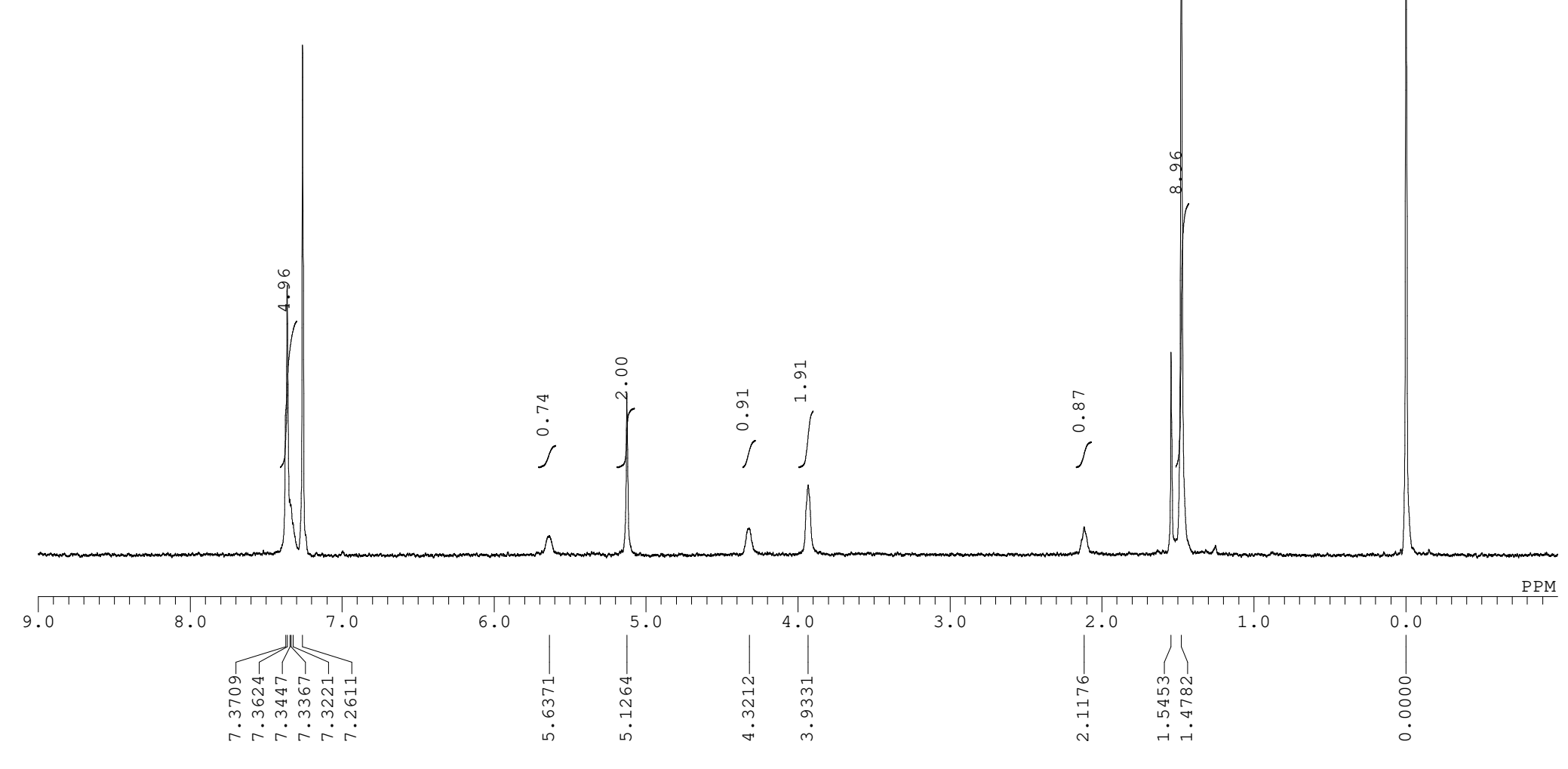

S30 


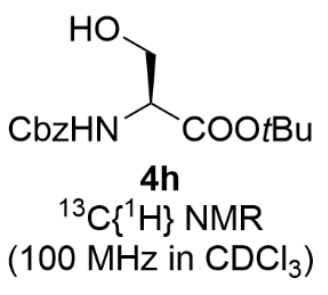

${ }^{13} \mathrm{C}\left\{{ }^{1} \mathrm{H}\right\}$ NMR spectrum of $\mathbf{4 h}$

$100 \mathrm{MHz}$ in $\left.\mathrm{CDCl}_{3}\right)$

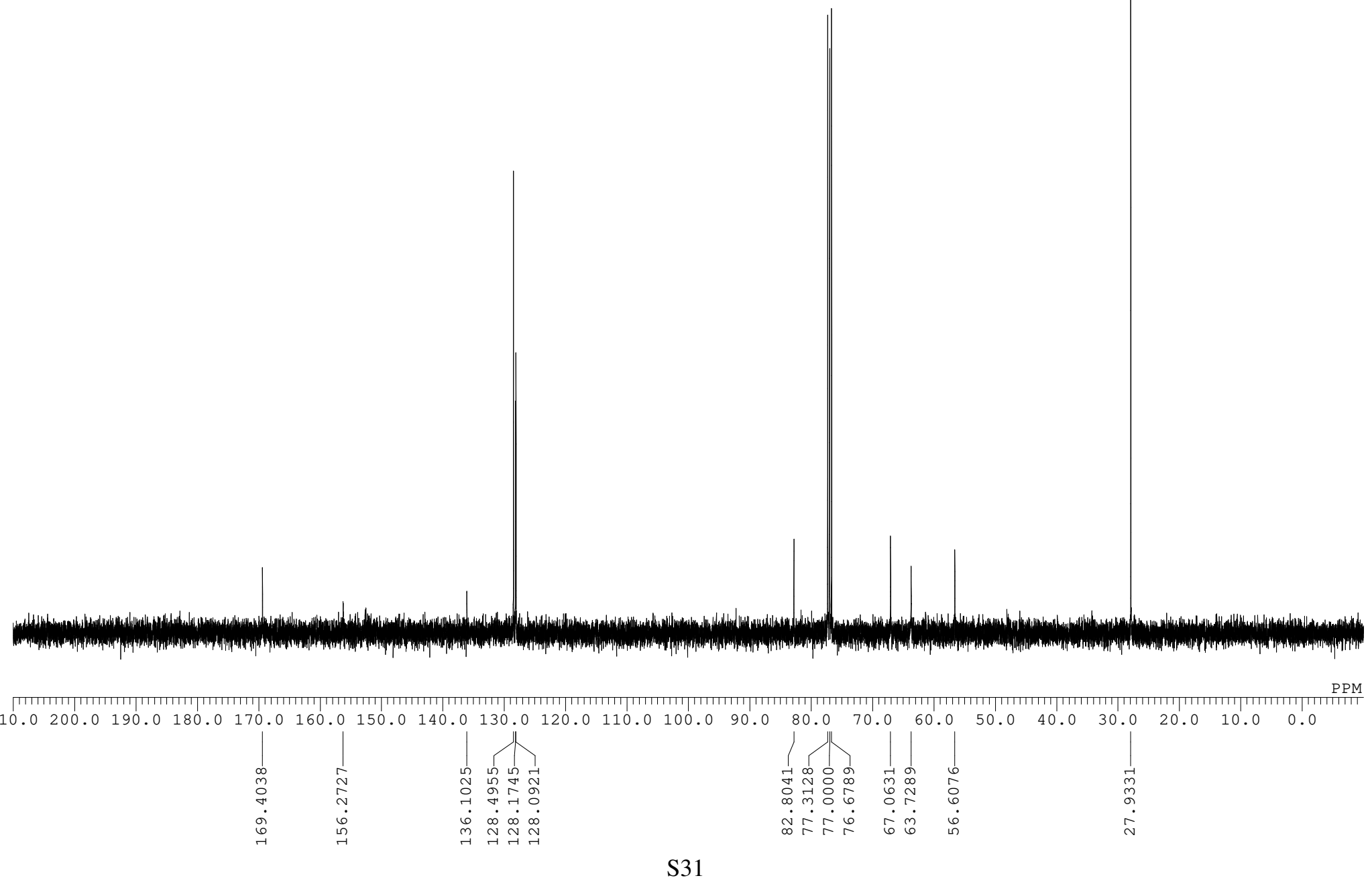



HO,
$\mathrm{CbzHN}$ COOtBu
$4 i$
${ }^{1} \mathrm{H}$ NMR
(400 MHz in $\mathrm{CDCl}_{3}$ )

${ }^{1} \mathrm{H}$ NMR spectrum of $\mathbf{4 i}$

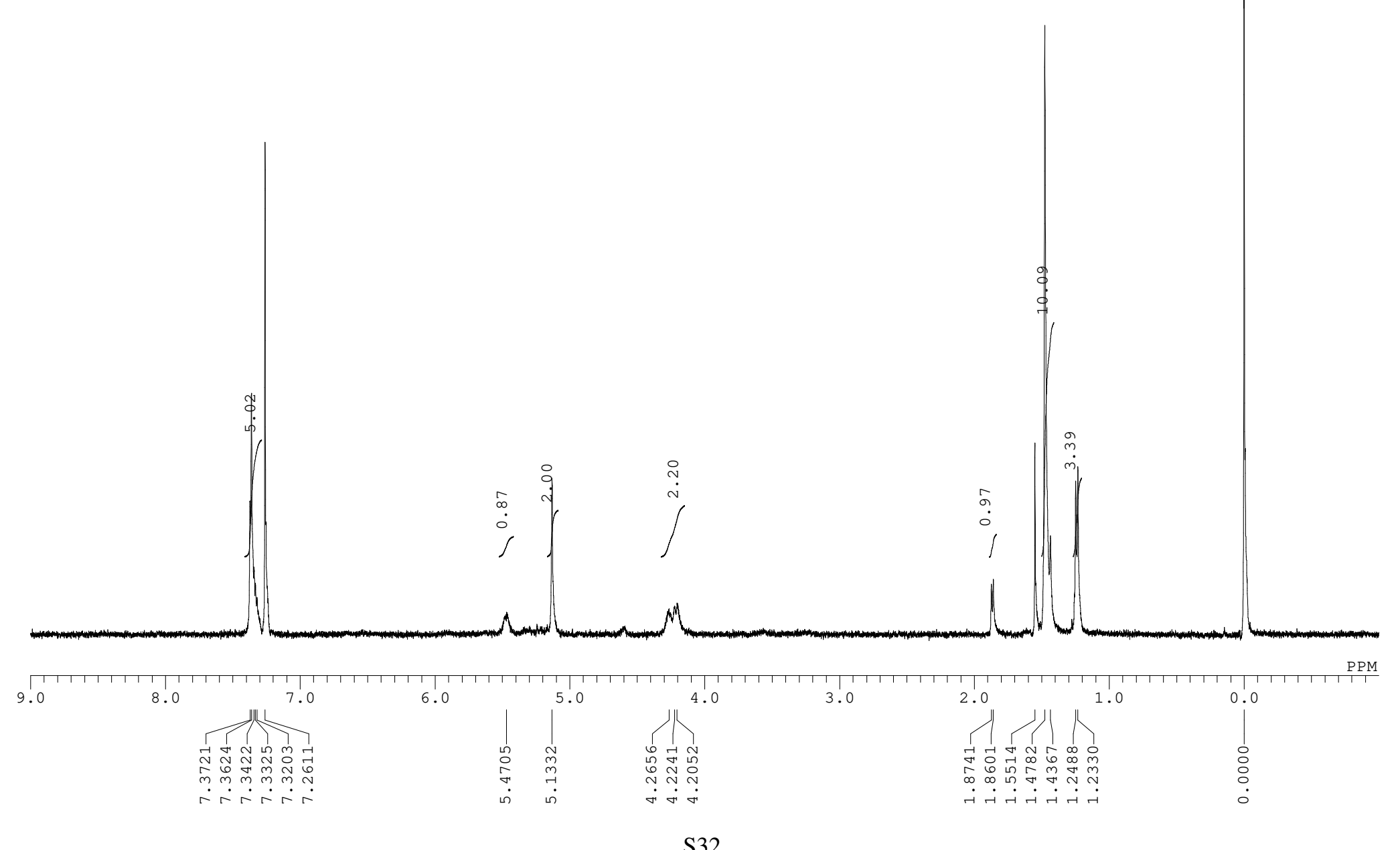




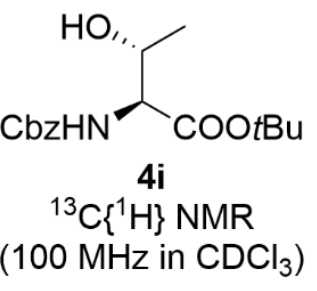

${ }^{13} \mathrm{C}\left\{{ }^{1} \mathrm{H}\right\}$ NMR spectrum of $\mathbf{4 i}$

(100 $\mathrm{MHz}$ in $\mathrm{CDCl}_{3}$ )

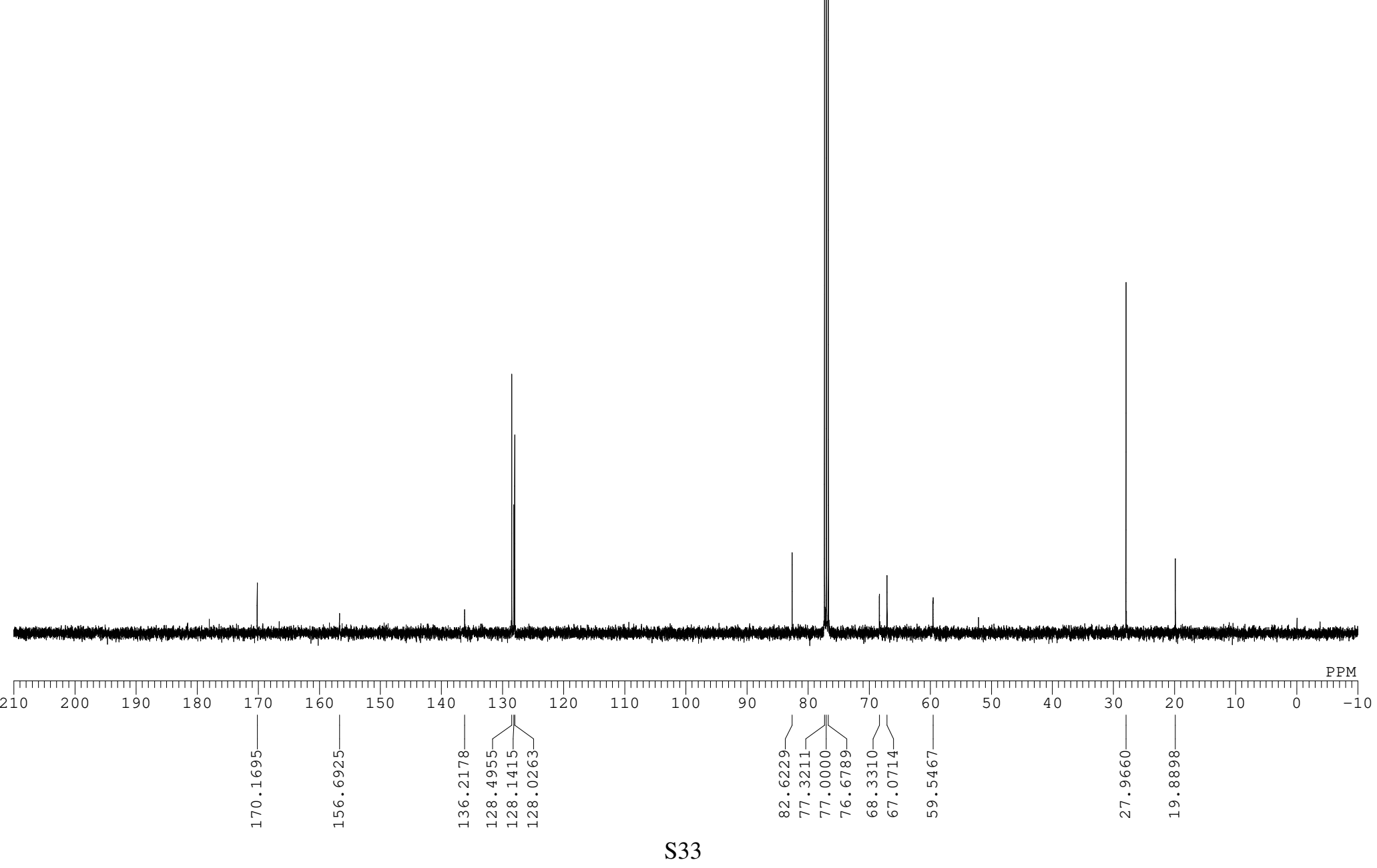




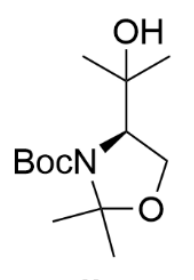

${ }^{1} \mathrm{H}$ NMR spectrum of $\mathbf{4 k}$

4k

${ }^{1} \mathrm{H}$ NMR

(600 MHz in $\mathrm{CDCl}_{3}$ )

NMR spectrum of $4 k$

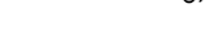

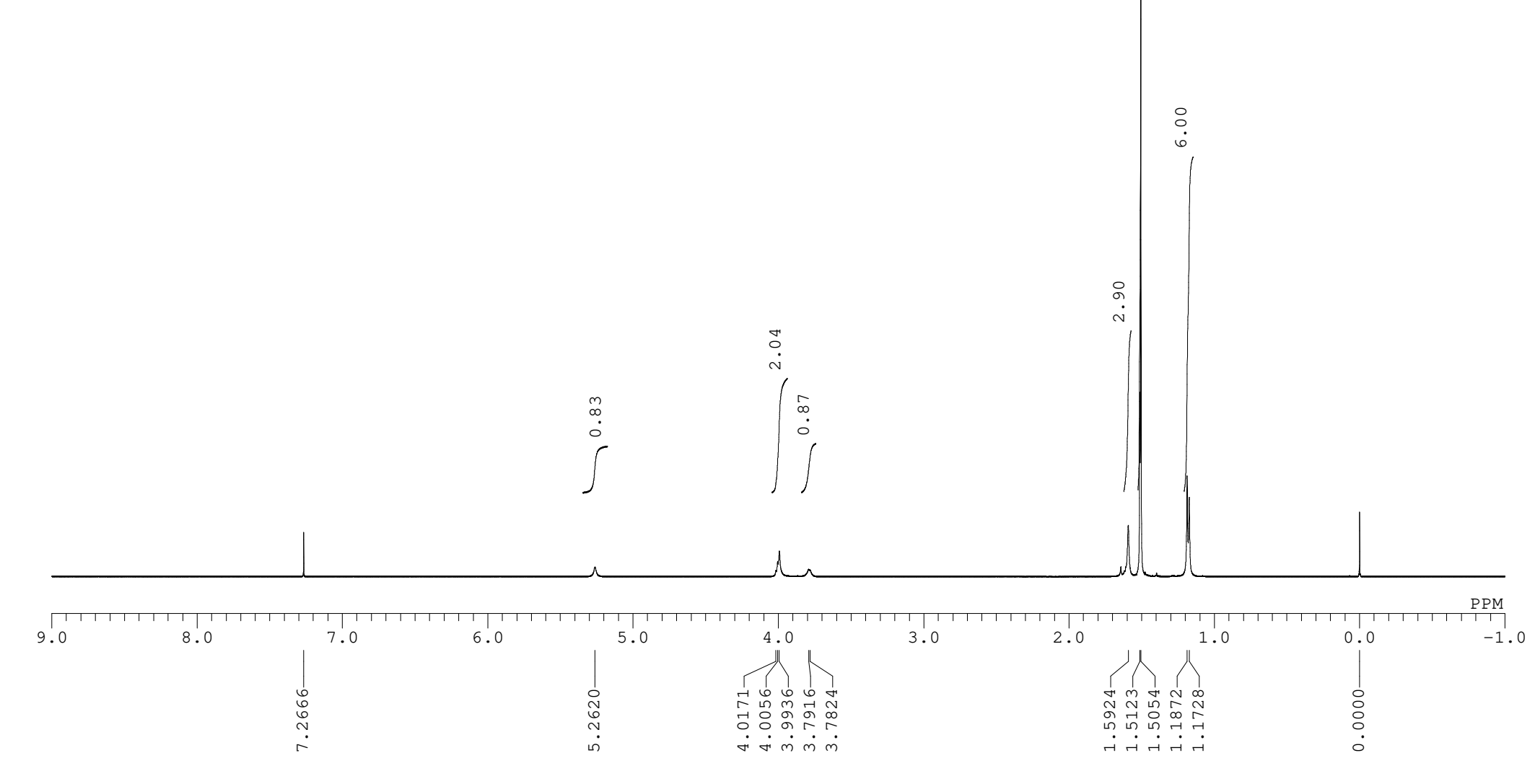




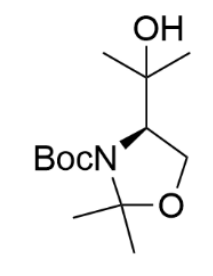

${ }^{13} \mathrm{C}\left\{{ }^{1} \mathrm{H}\right\}$ NMR spectrum of $\mathbf{4 k}$

${ }^{13} \mathrm{C}\left\{{ }^{1} \mathrm{H}\right\}$ NMR

$\left(150 \mathrm{MHz}^{\text {in } \mathrm{CDCl}_{3}}\right.$ )

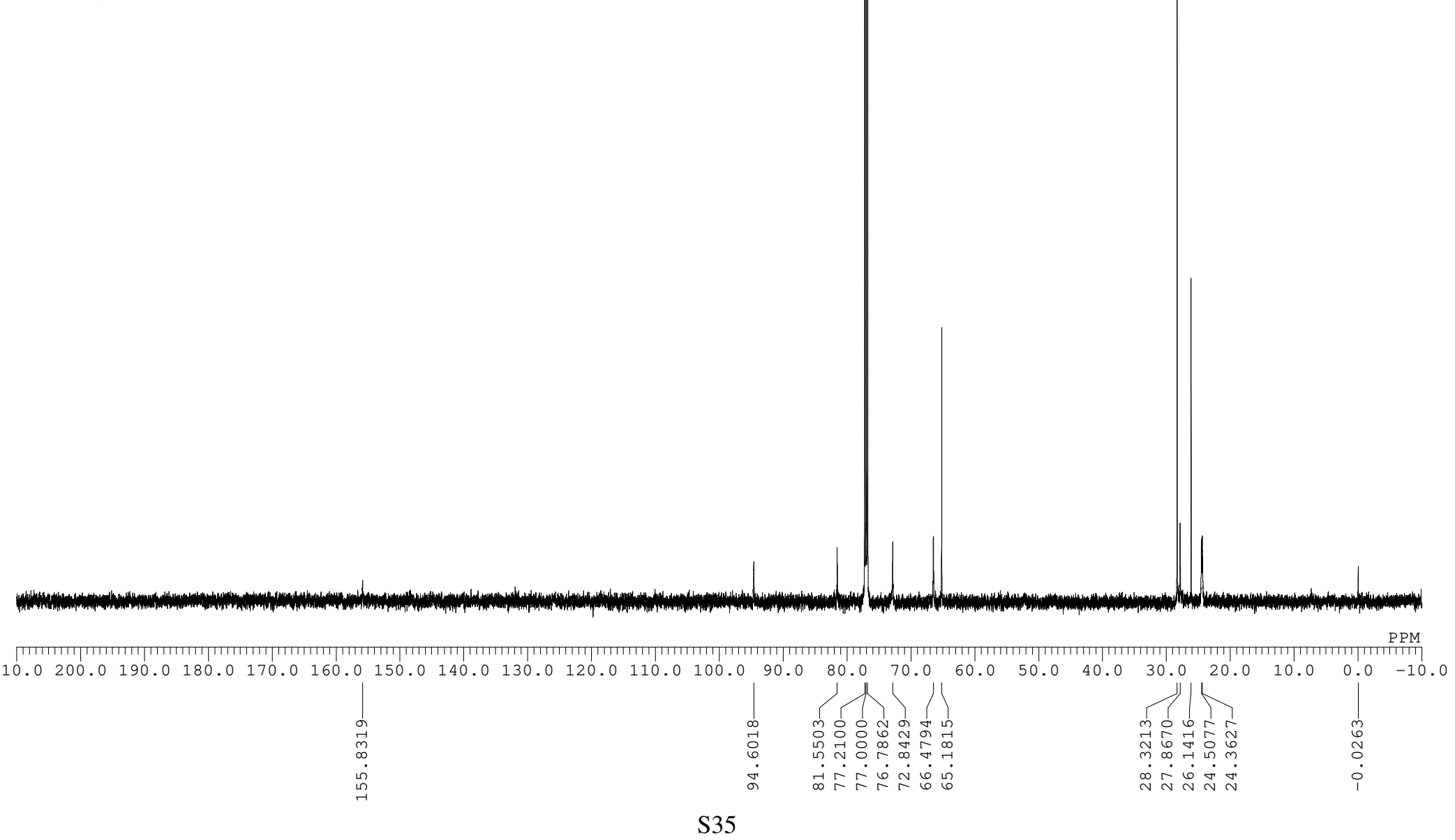




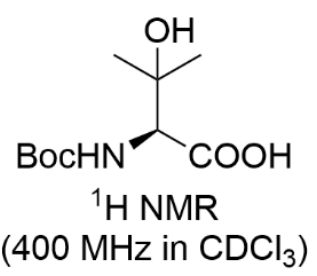

${ }^{1} \mathrm{H}$ NMR spectrum of Boc-Val(3-OH)-OH

${ }^{1} \mathrm{H} \mathrm{NMR}$
$\left(400 \mathrm{MHz}\right.$ in $\left.\mathrm{CDCl}_{3}\right)$

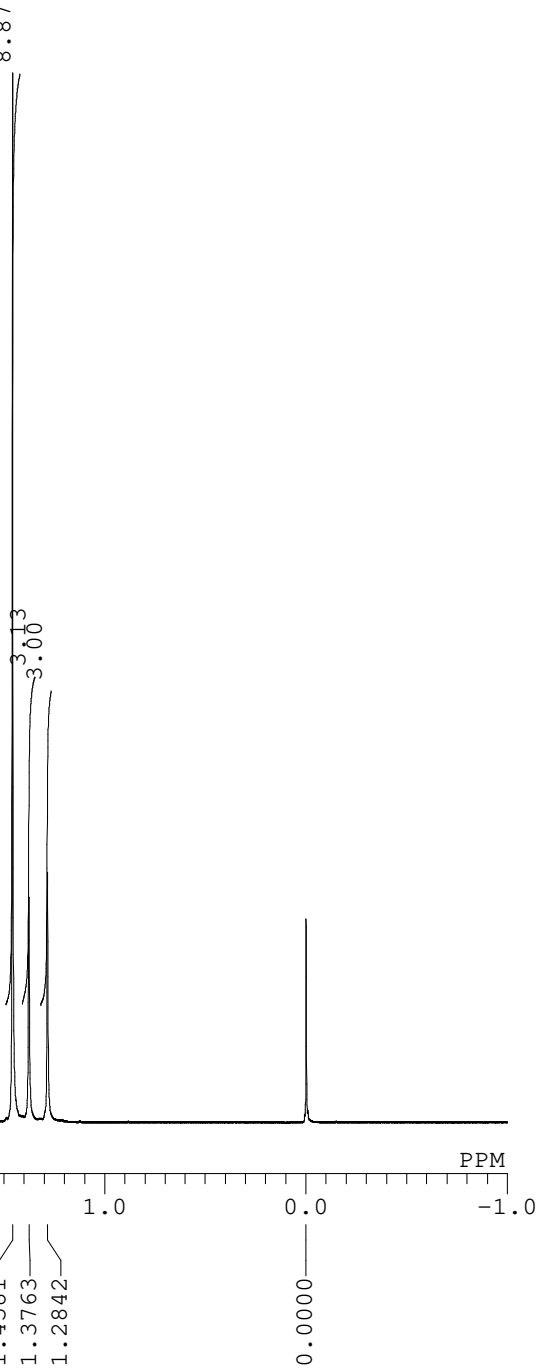


$\mathrm{OH}$

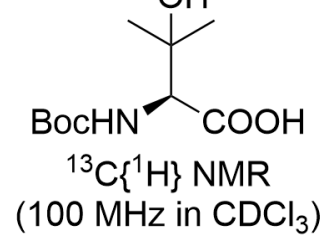

${ }^{13} \mathrm{C}\left\{{ }^{1} \mathrm{H}\right\} \mathrm{NMR}$
${ }^{13} \mathrm{C}\left\{{ }^{1} \mathrm{H}\right\}$ NMR spectrum of Boc-Val(3-OH)-OH

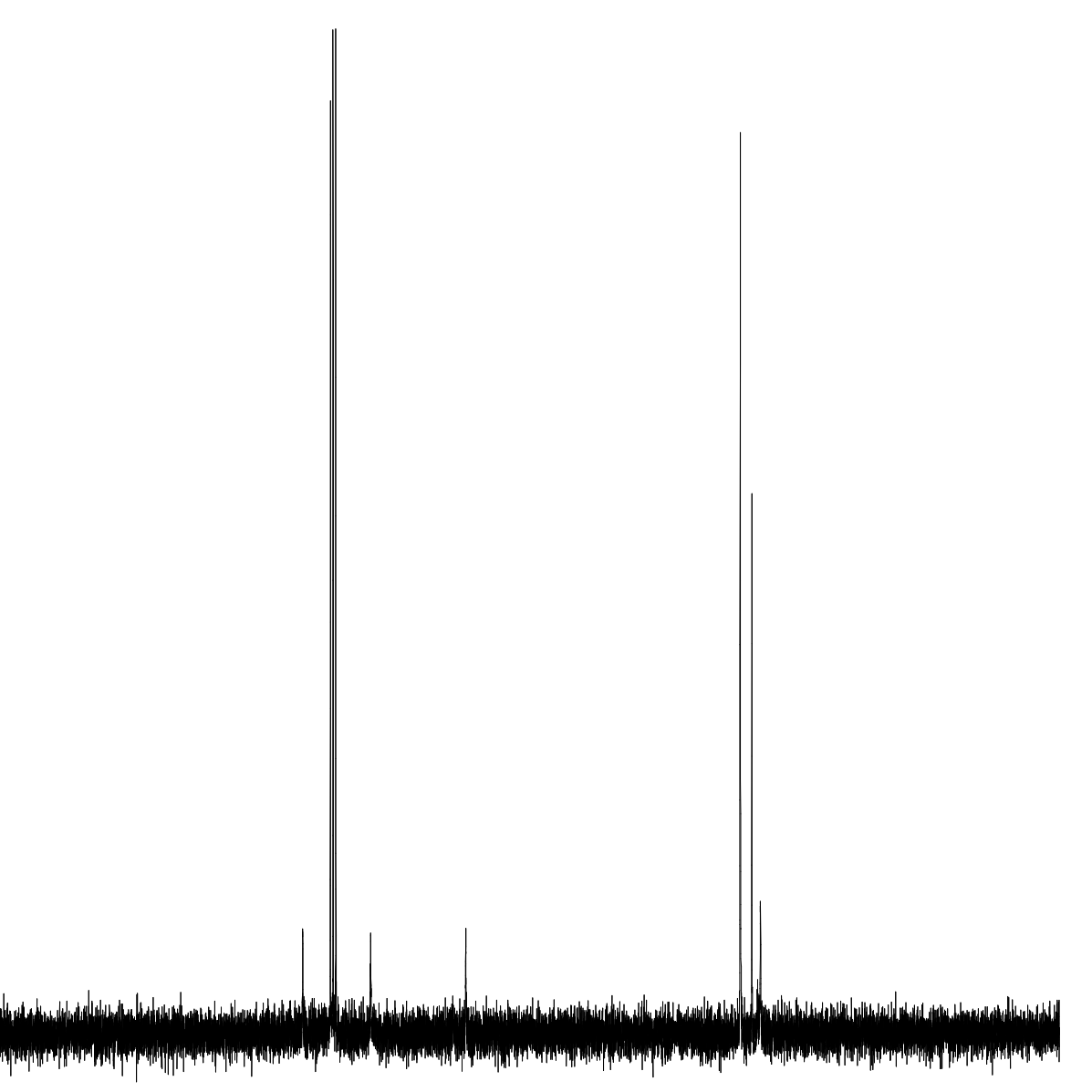

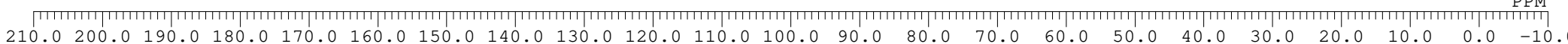

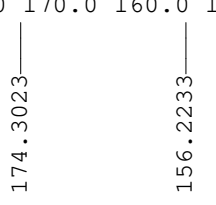
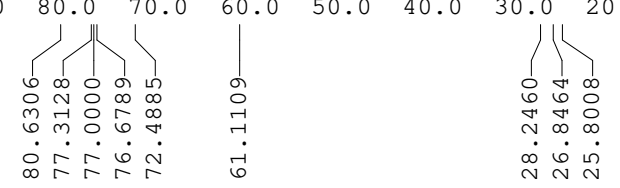


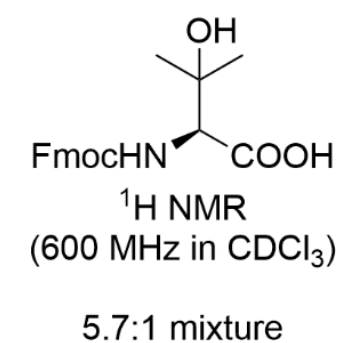

5.7:1 mixture

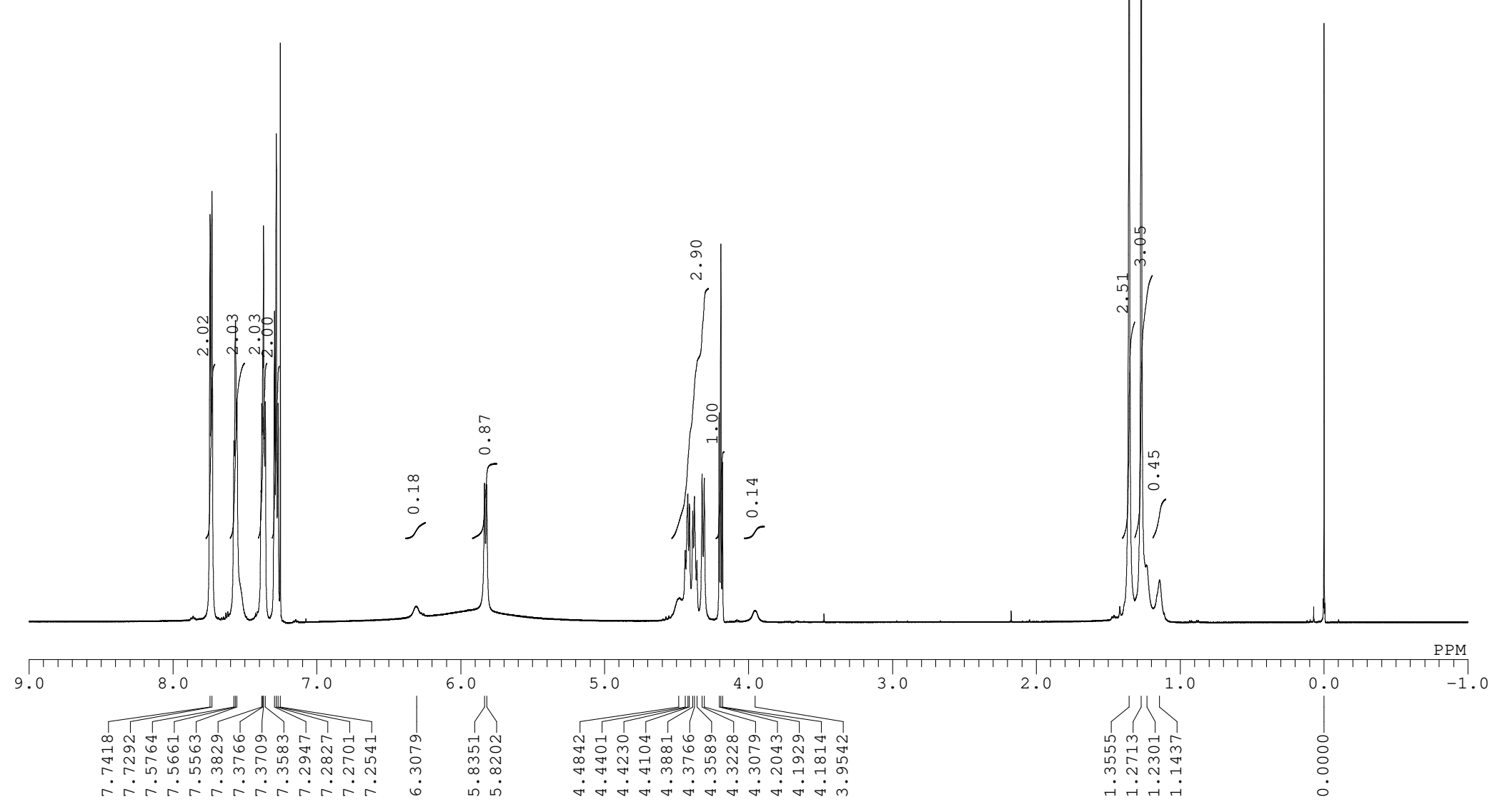




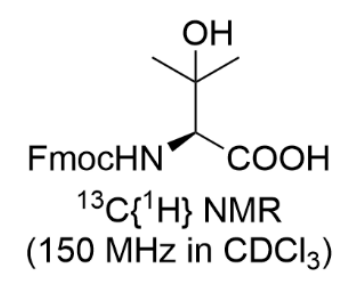

${ }^{13} \mathrm{C}\left\{{ }^{1} \mathrm{H}\right\}$ NMR spectrum of Fmoc-Val(3-OH)-OH

\section{${ }^{13} \mathrm{C}\left\{{ }^{1} \mathrm{H}\right\}$ NMR}

$150 \mathrm{MHz}$ in $\mathrm{CDCl}_{3}$ )

5.7:1 mixture 
$\underbrace{\mathrm{OH}}_{\text {COOtBu }}$

$4 \mathrm{j}$

${ }^{1} \mathrm{H} N M R$

$\left(600 \mathrm{MHz}\right.$ in $\mathrm{CDCl}_{3}$ )
${ }^{1} \mathrm{H}$ NMR spectrum of $\mathbf{4} \mathbf{j}$

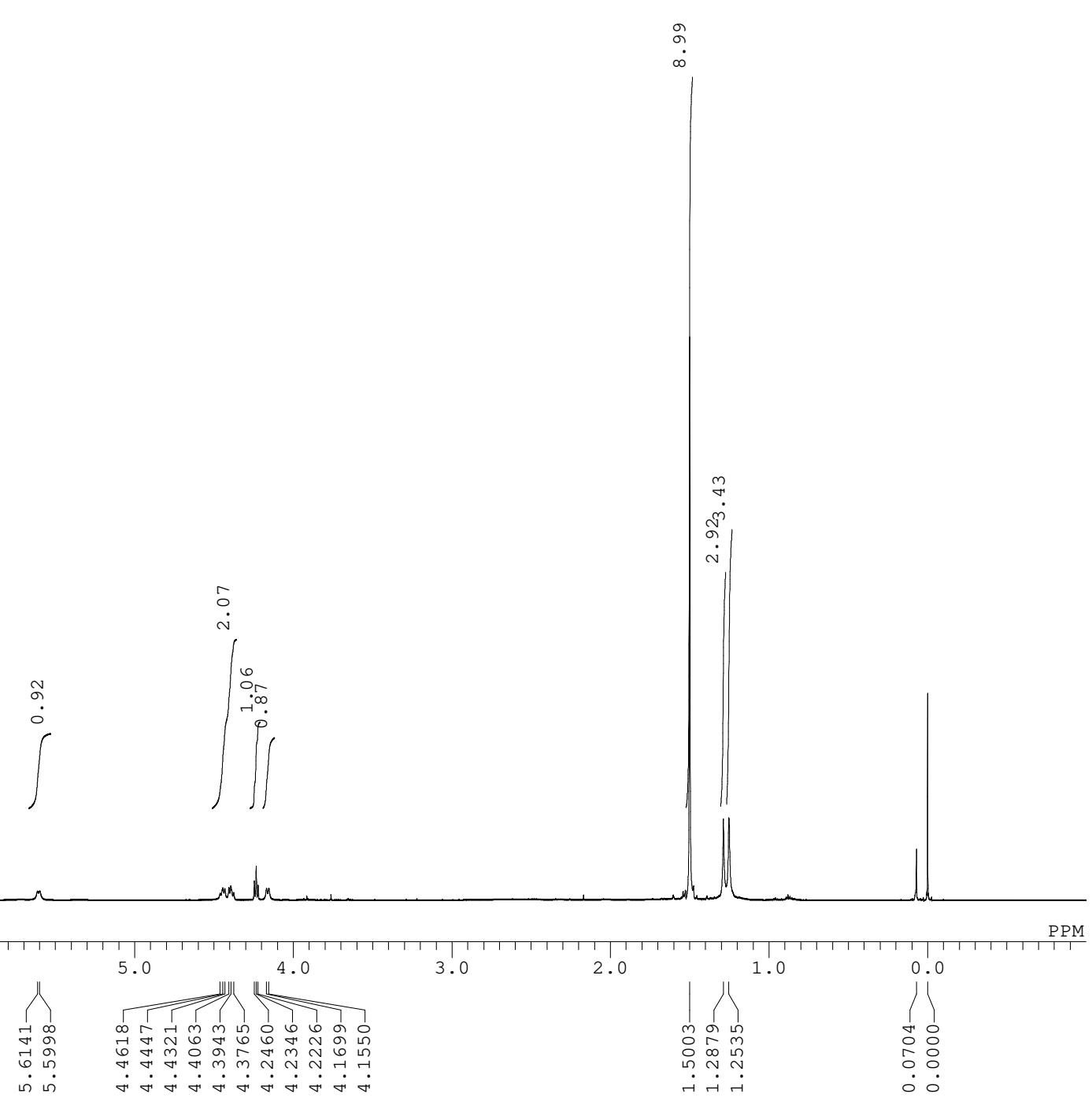

$\mathrm{S} 40$ 
FmochN COOtBu

${ }^{13} \mathrm{C}\left\{{ }^{1} \mathrm{H}\right\}$ NMR

$\left(150 \mathrm{MHz}\right.$ in $\left.\mathrm{CDCl}_{3}\right)$

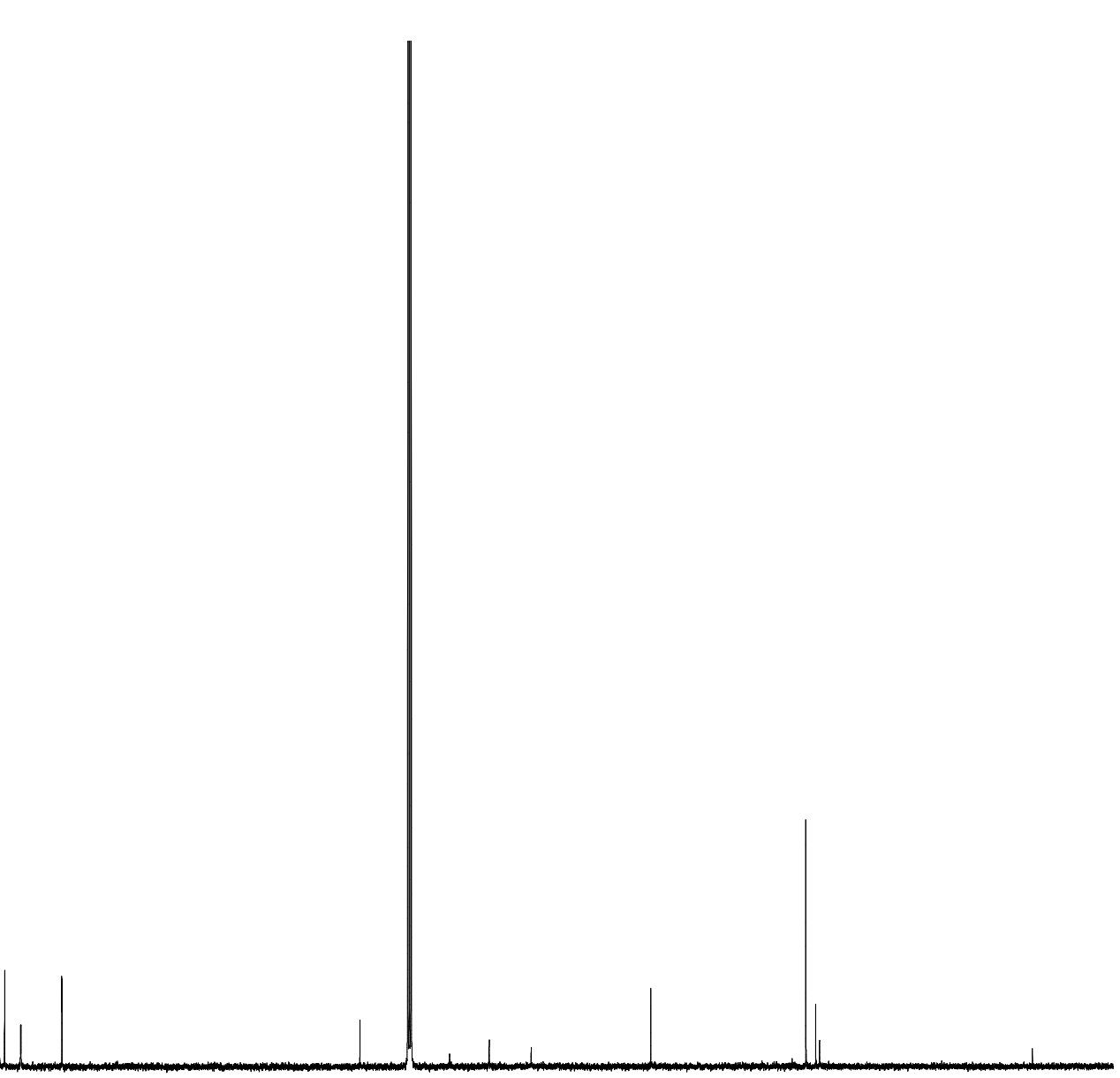

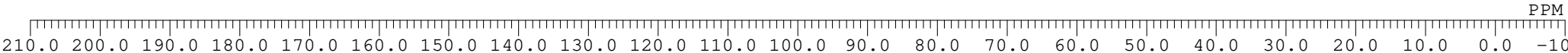

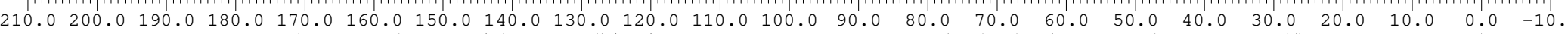

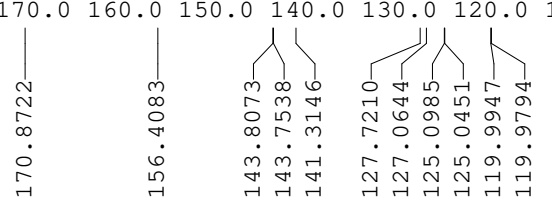




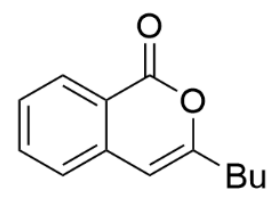

${ }^{1}$ H NMR spectrum of $\mathbf{6}$

6
$1 \mathrm{H} N M R$

$\left(600 \mathrm{MHz}\right.$ in $\mathrm{CDCl}_{3}$ )

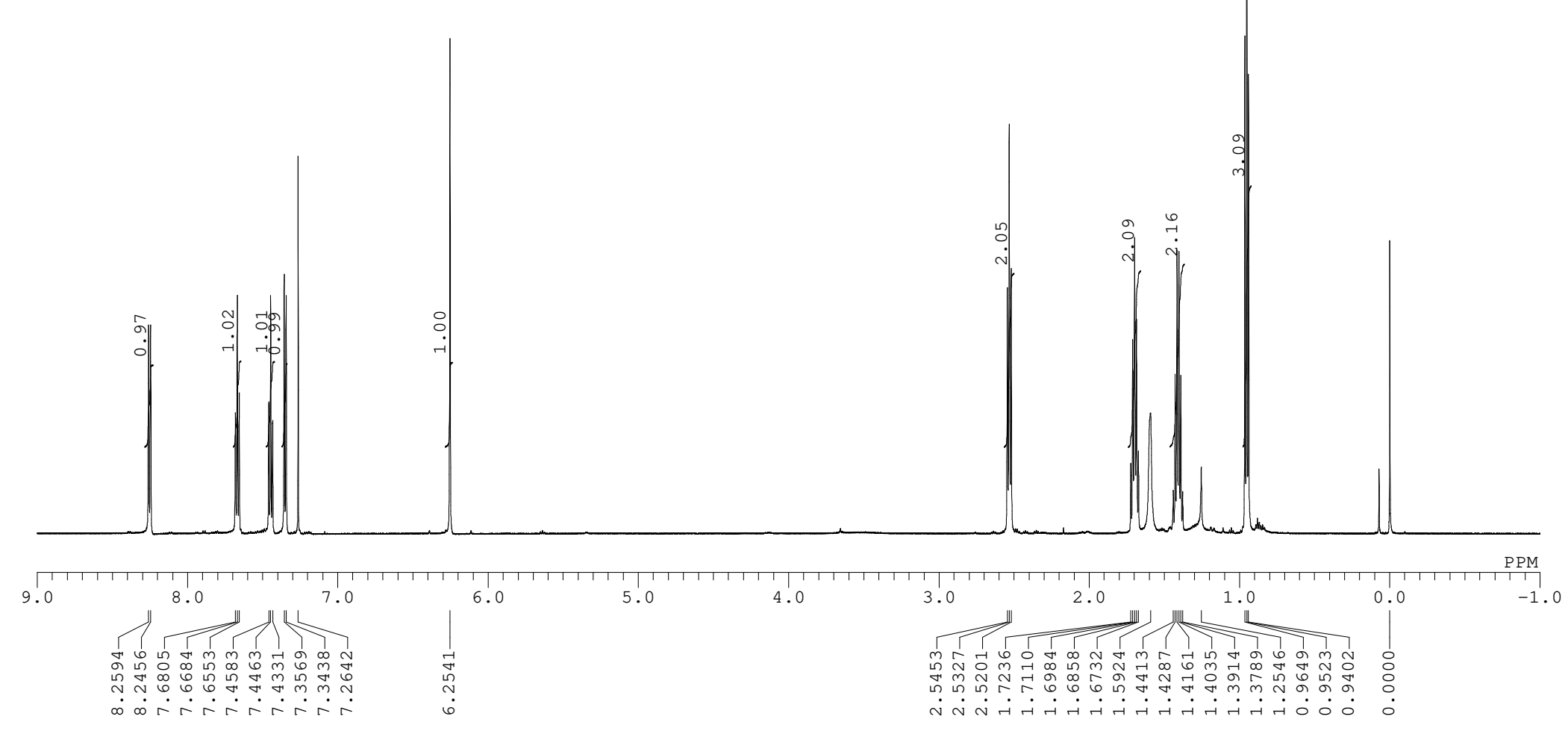




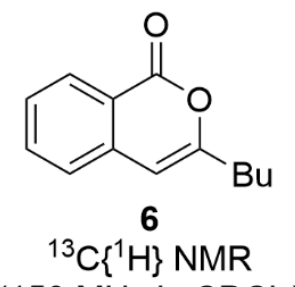

${ }^{13} \mathrm{C}\left\{{ }^{1} \mathrm{H}\right\}$ NMR spectrum of 6

(150 $\mathrm{MHz}$ in $\mathrm{CDCl}_{3}$ ) 


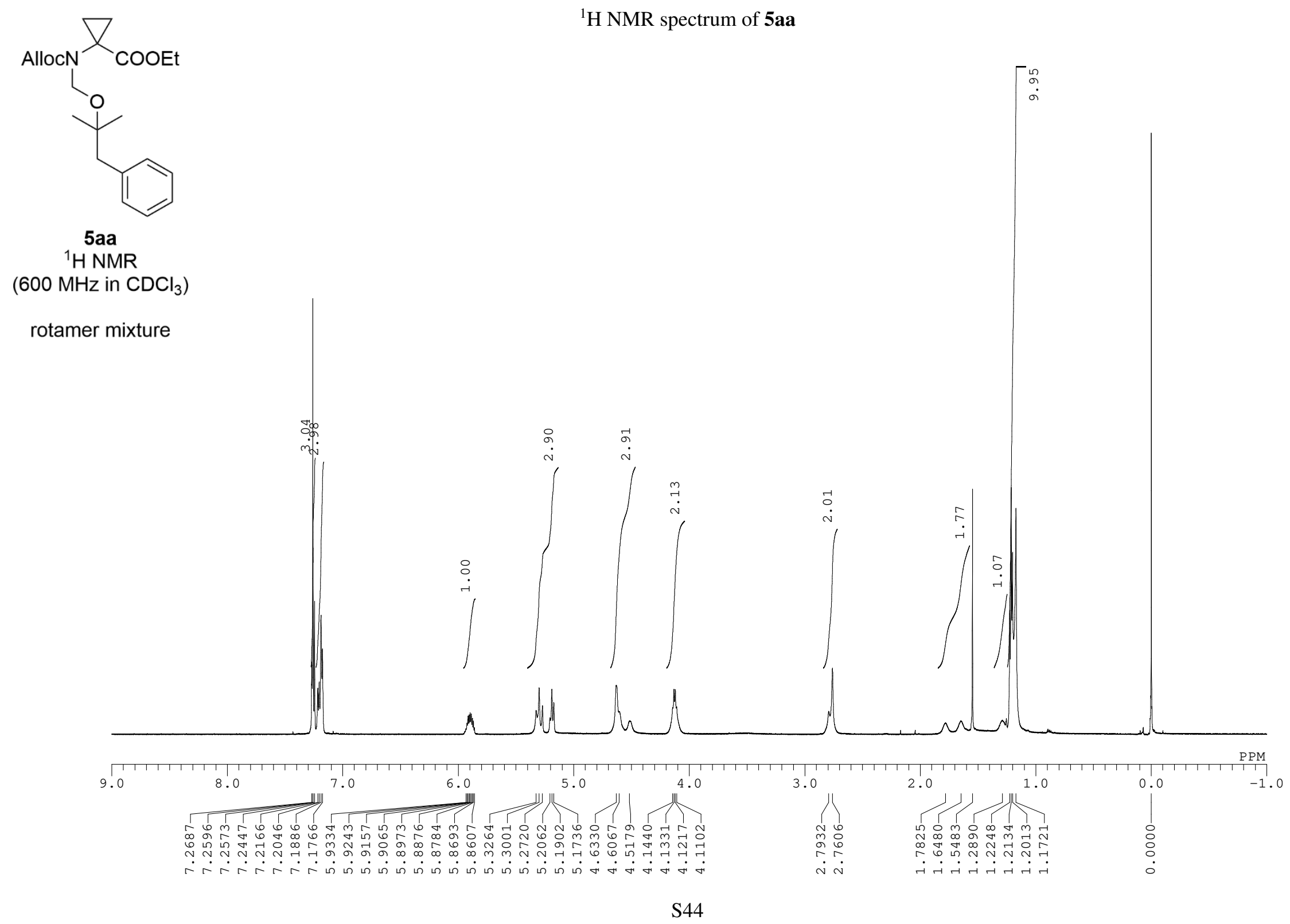




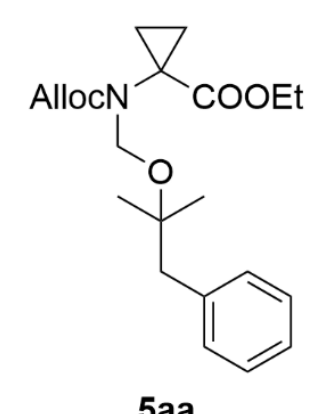

${ }^{13} \mathrm{C}\left\{{ }^{1} \mathrm{H}\right\}$ NMR spectrum of 5 aa

${ }^{13} \mathrm{C}\left\{{ }^{1} \mathrm{H}\right\}$ NMR

(150 $\mathrm{MHz}$ in $\mathrm{CDCl}_{3}$ )

rotamer mixture

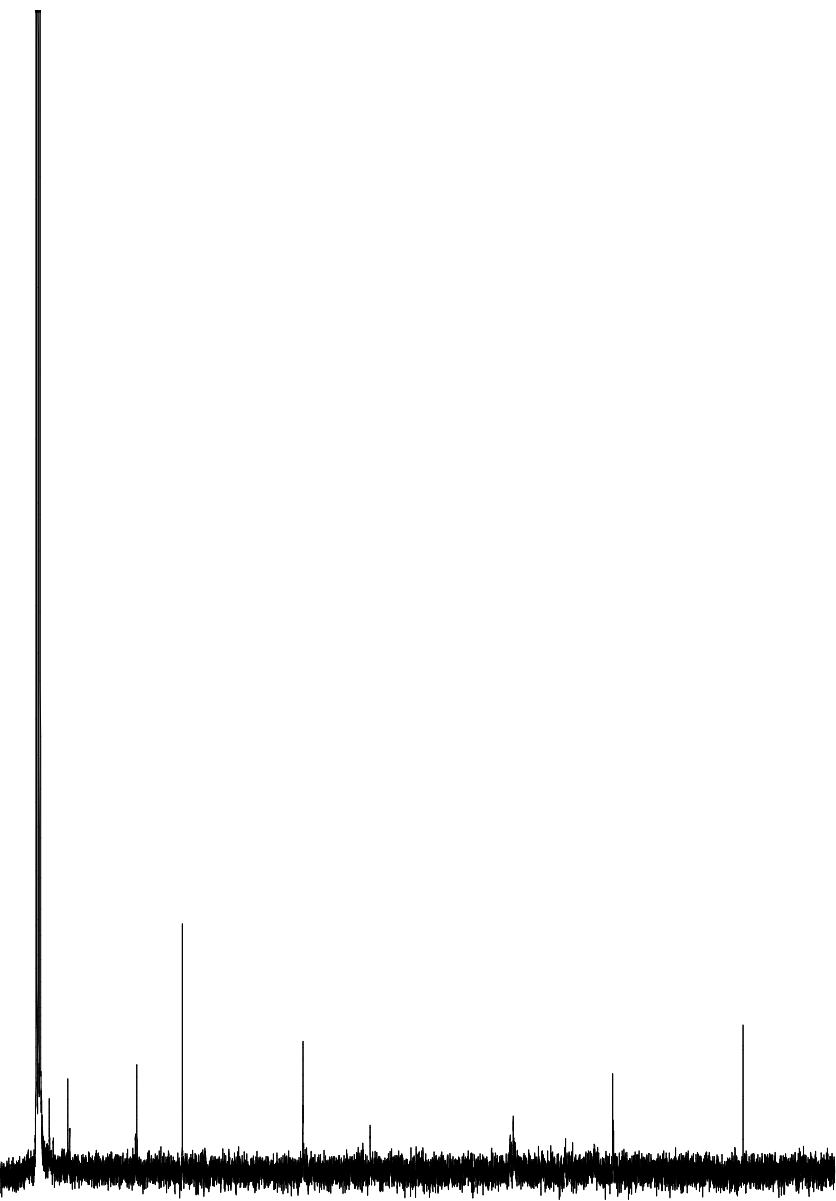

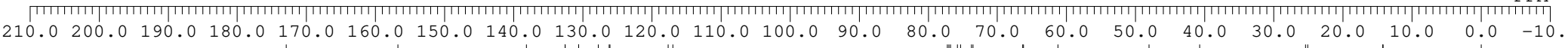

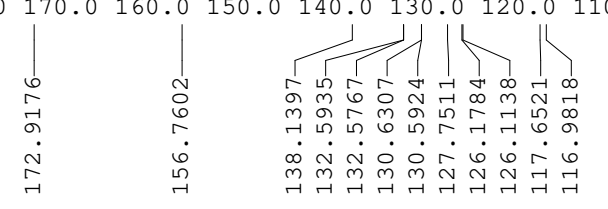

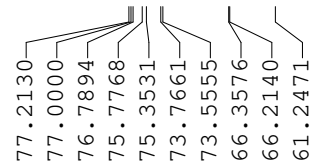

$\begin{array}{ll} & \\ 2 & 0 \\ 0 & 0 \\ 0 & 0 \\ 0 & 7 \\ 0 & 0 \\ \infty & 0 \\ \pi & 0\end{array}$ 


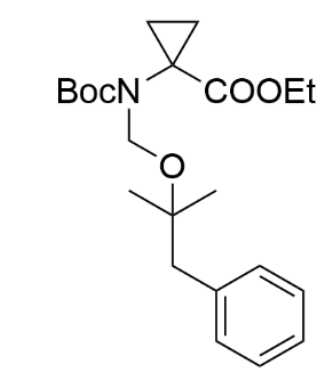

${ }^{1} \mathrm{H}$ NMR spectrum of $\mathbf{5} \mathbf{b a}$

5 ba

${ }^{1} \mathrm{H}$ NMR

$\left(600 \mathrm{MHz}\right.$ in $\mathrm{CDCl}_{3}$ )

rotamer mixture

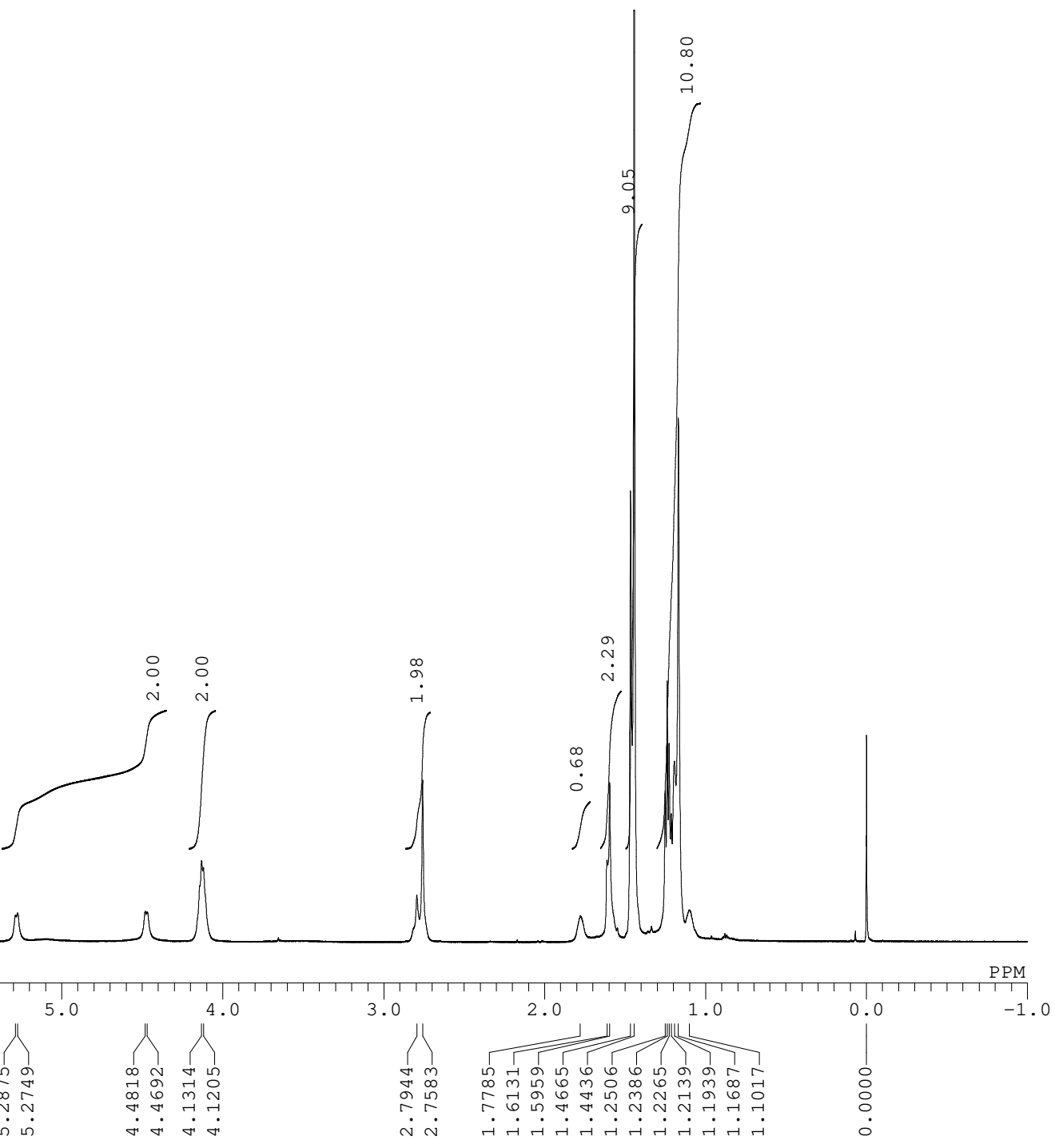




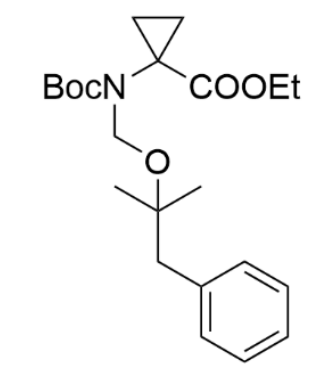

${ }^{13} \mathrm{C}\left\{{ }^{1} \mathrm{H}\right\}$ NMR spectrum of $\mathbf{5 b a}$

5 ba

${ }^{13} \mathrm{C}\left\{{ }^{1} \mathrm{H}\right\}$ NMR

$\left(150 \mathrm{MHz}\right.$ in $\left.\mathrm{CDCl}_{3}\right)$

rotamer mixture

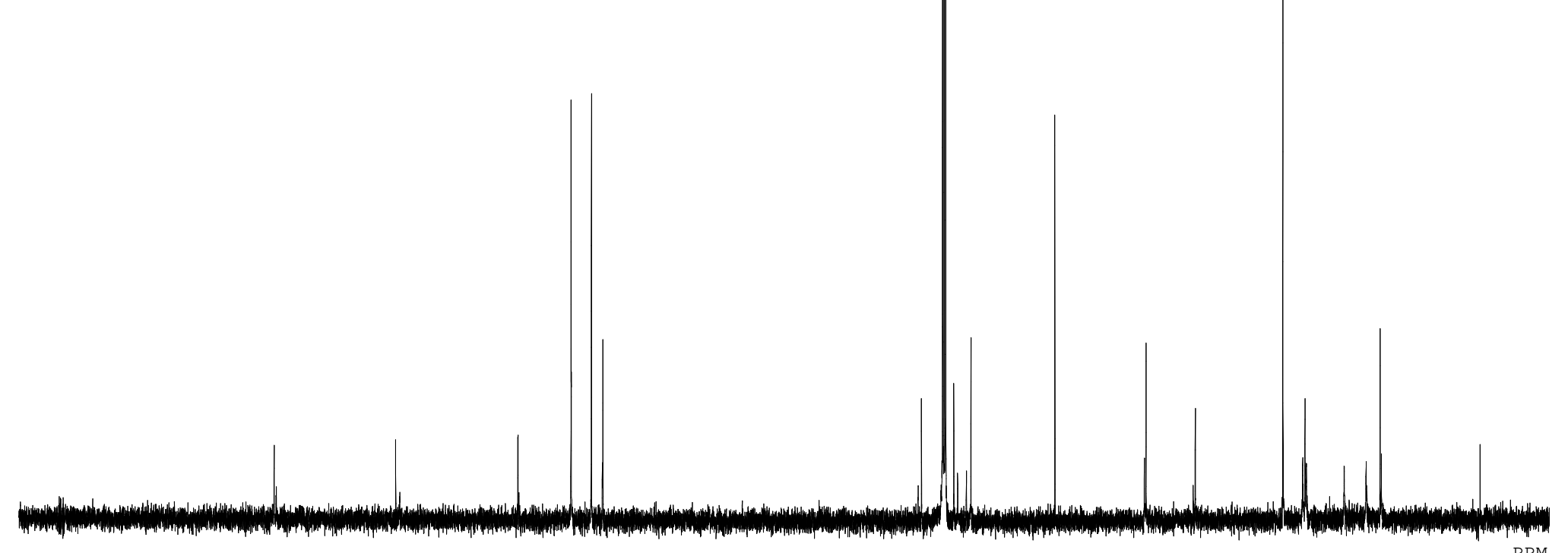
210.0200 .0190 .0180 .0170 .0160 .0150 .0140 .0130 .0120 .0110 .0100 .090 .0
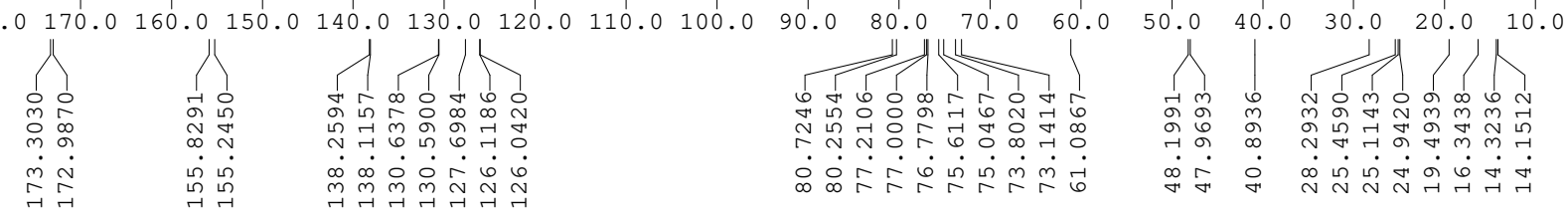


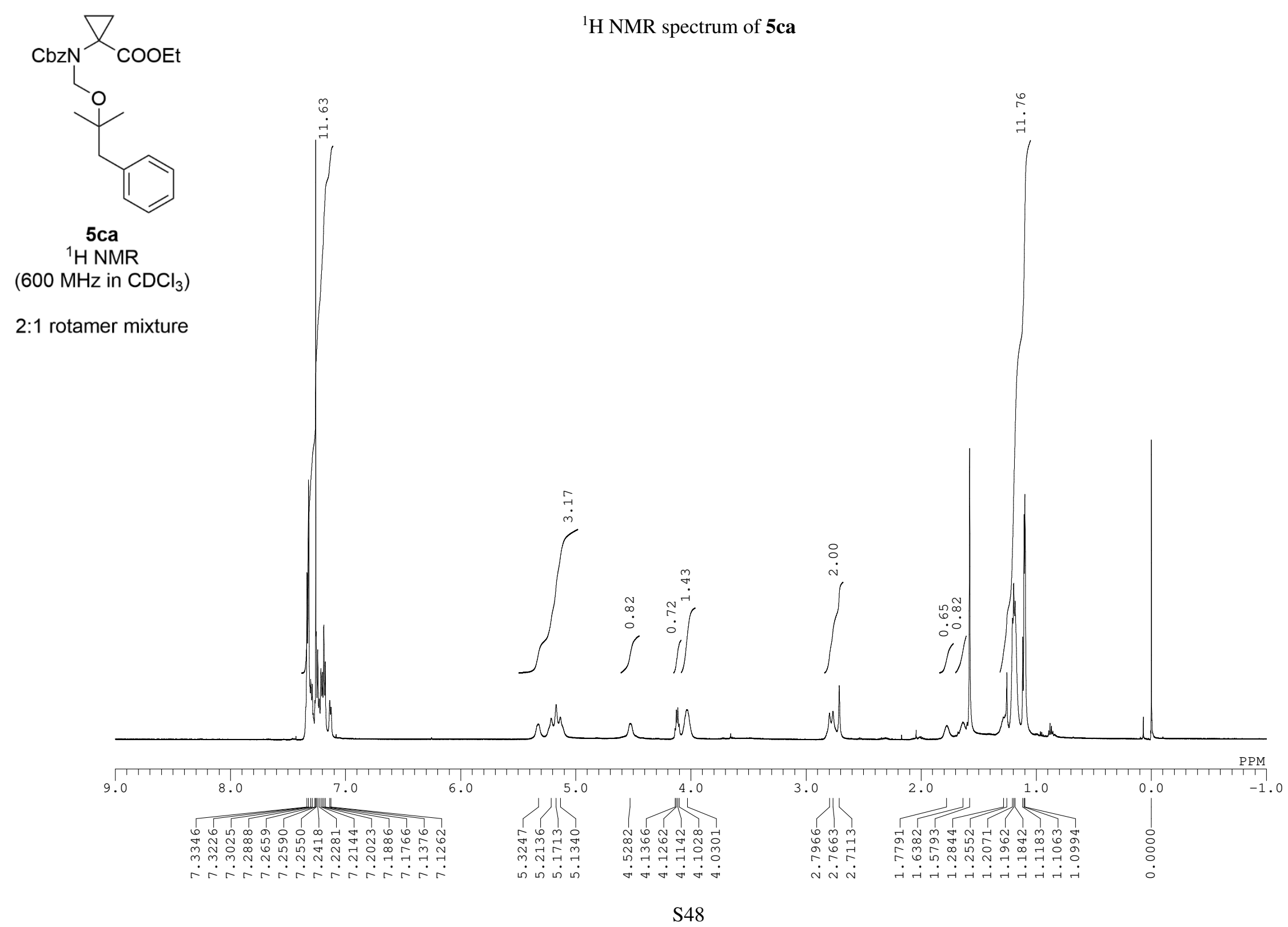




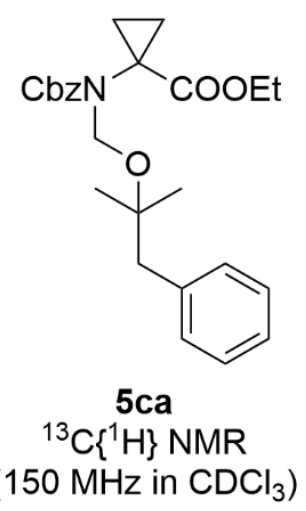

${ }^{13} \mathrm{C}\left\{{ }^{1} \mathrm{H}\right\}$ NMR spectrum of $\mathbf{5} \mathbf{c a}$

$2: 1$ rotamer mixture

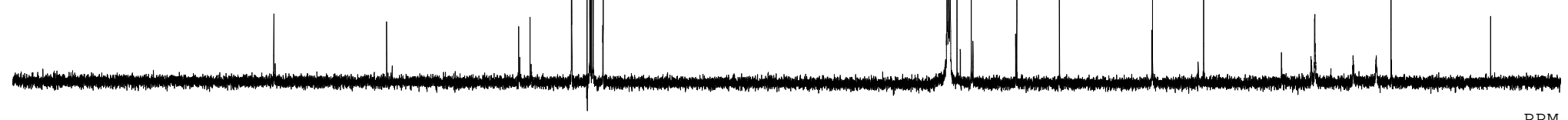

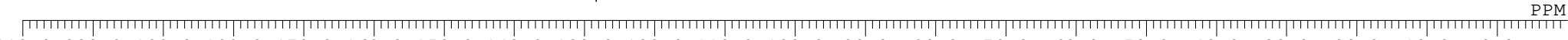
$210.0200 .0190 .0180 .0170 .0160 .0150 .0140 .0130 .0120 .0110 .0100 .0 \quad 90.0$
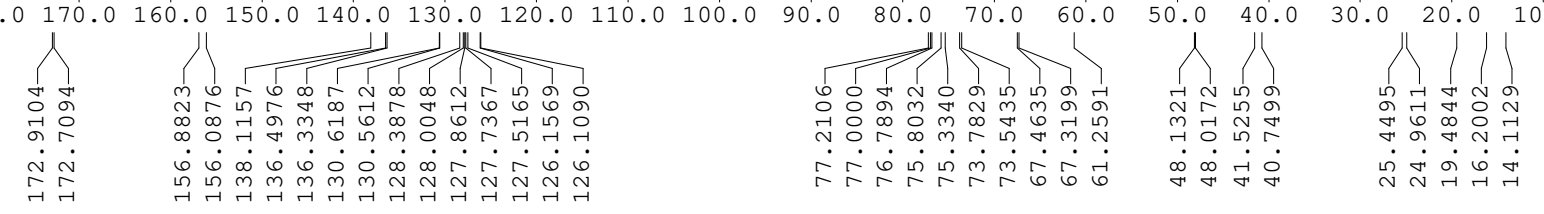


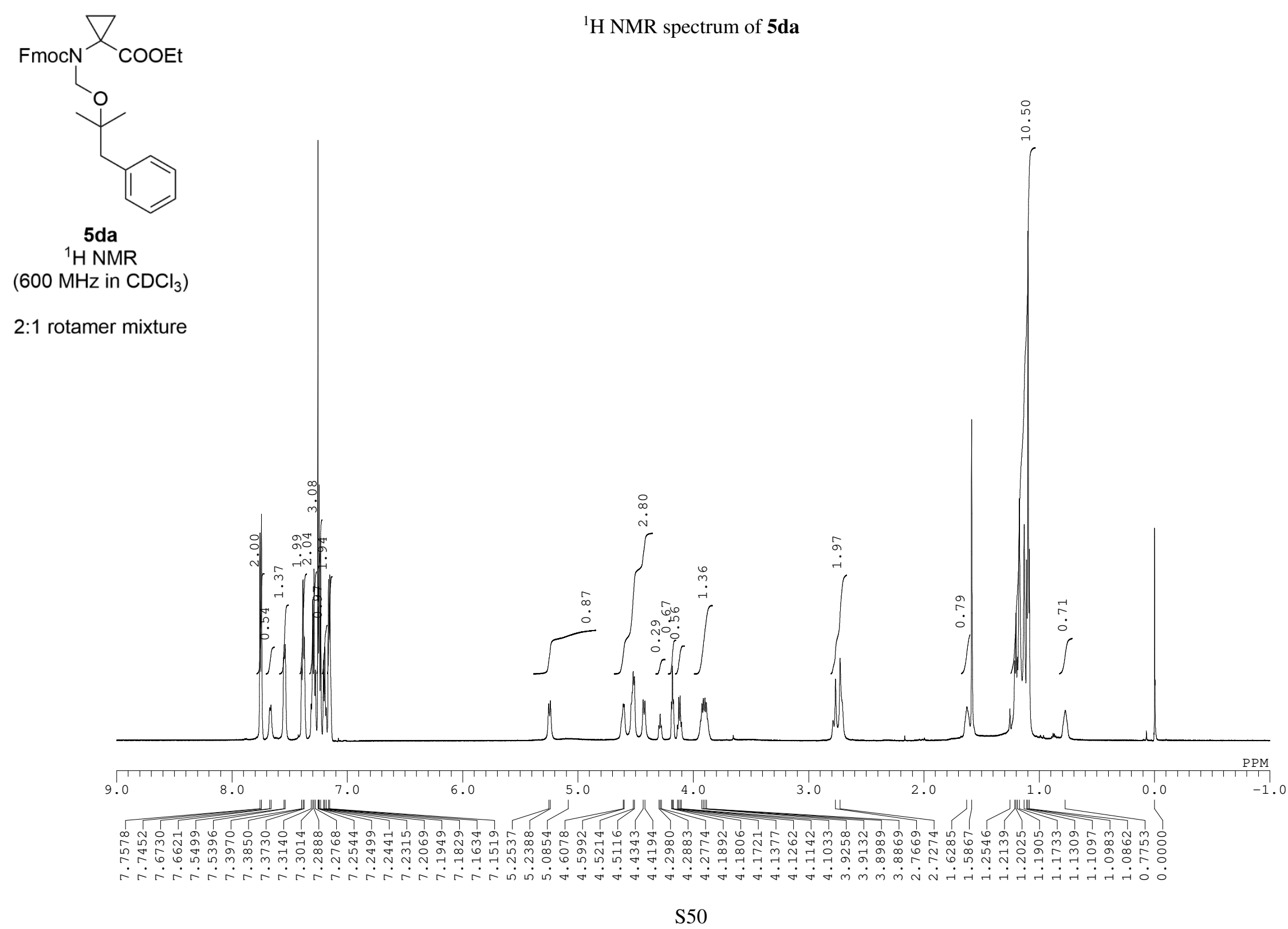




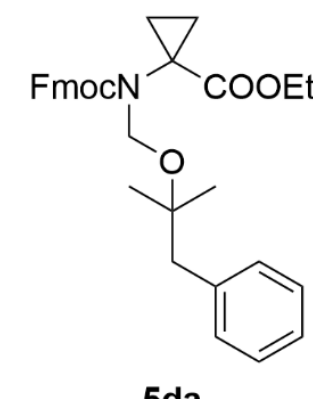

${ }^{13} \mathrm{C}\left\{{ }^{1} \mathrm{H}\right\}$ NMR spectrum of $\mathbf{5} \mathbf{d a}$

$5 d a$

${ }^{13} \mathrm{C}\left\{{ }^{1} \mathrm{H}\right\}$ NMR

(150 $\mathrm{MHz}$ in $\mathrm{CDCl}_{3}$ )

$2: 1$ rotamer mixture

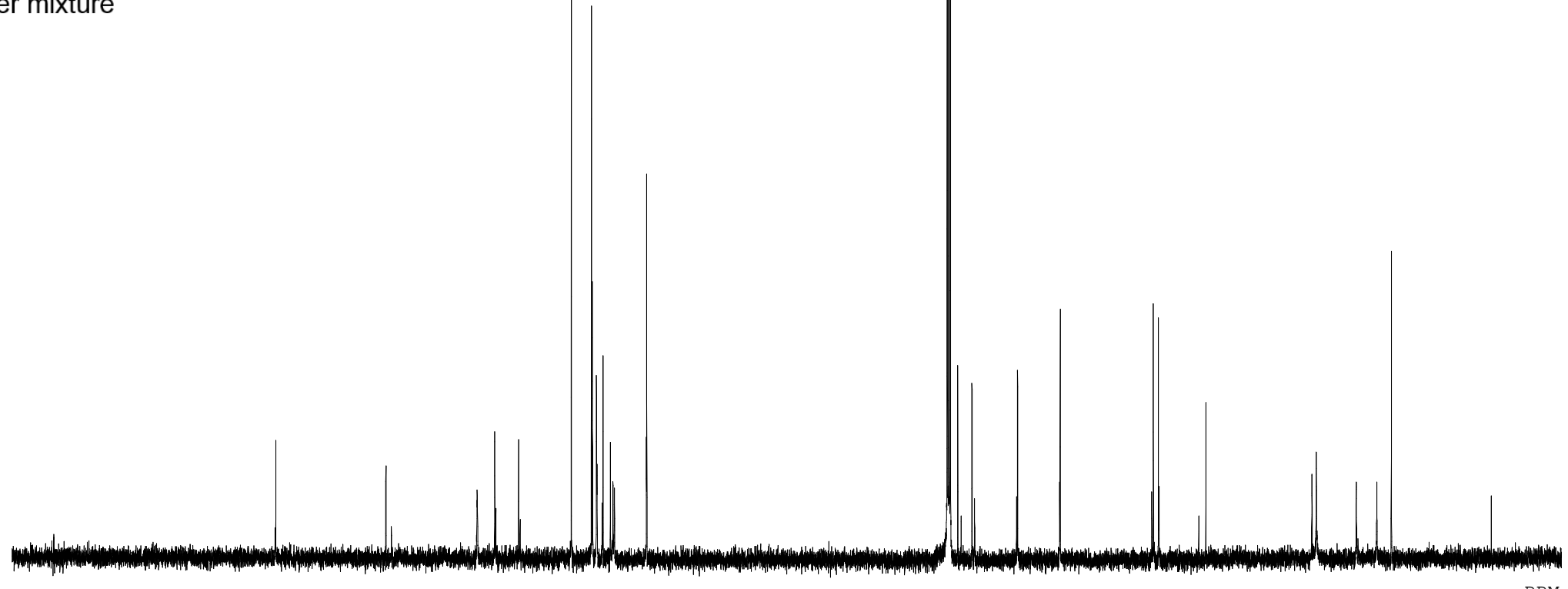

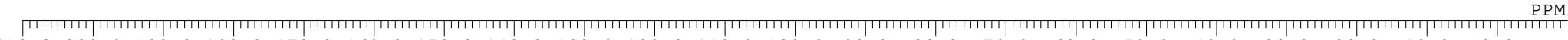
210.0200 .0190 .0180 .0170
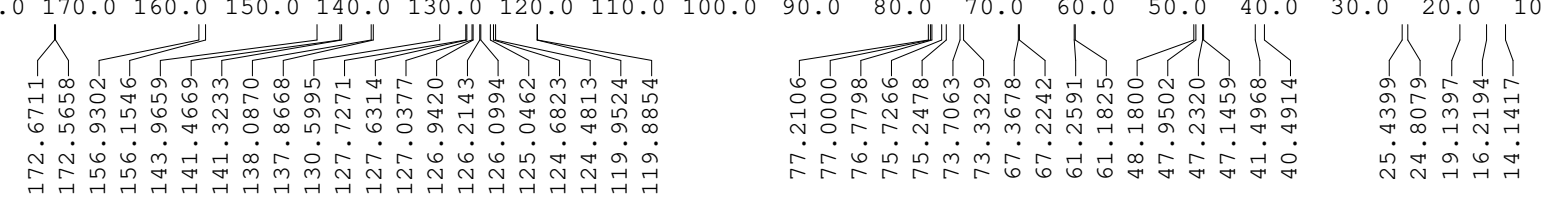


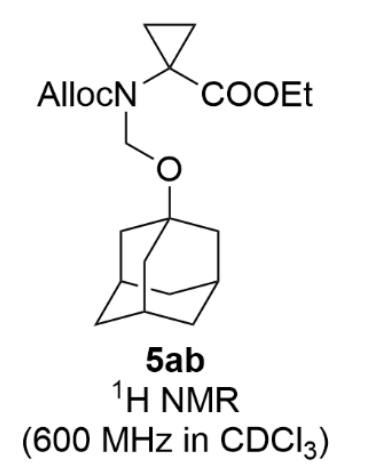

${ }^{1} \mathrm{H}$ NMR spectrum of $\mathbf{5 a b}$

rotamer mixture

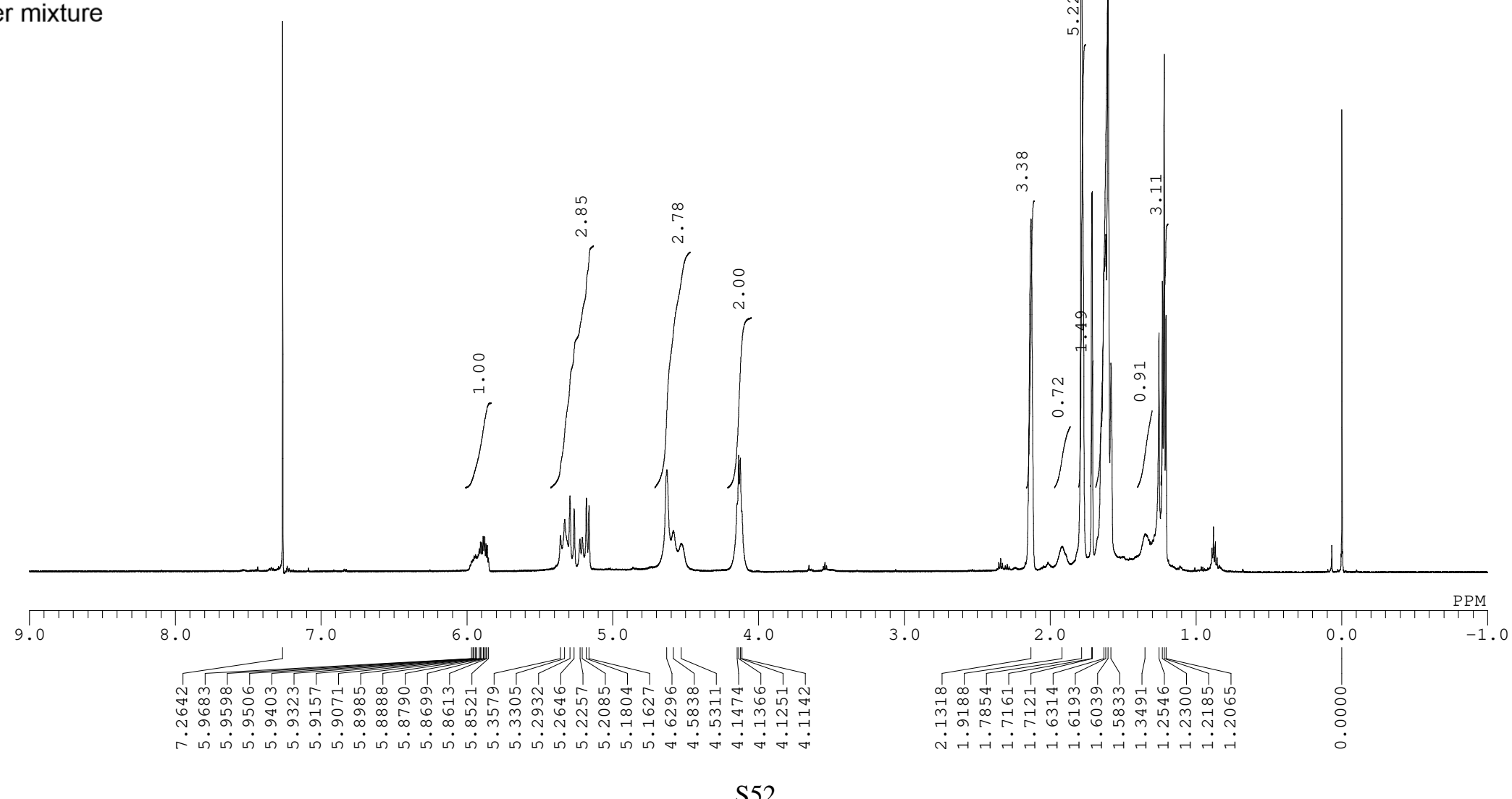




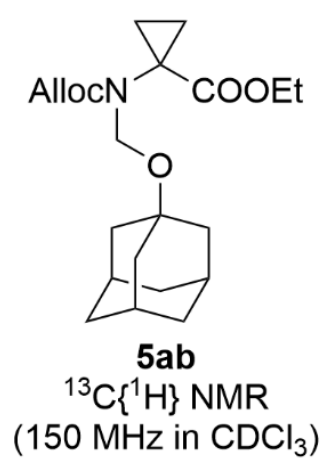

${ }^{13} \mathrm{C}\left\{{ }^{1} \mathrm{H}\right\}$ NMR spectrum of $\mathbf{5 a b}$

rotamer mixture

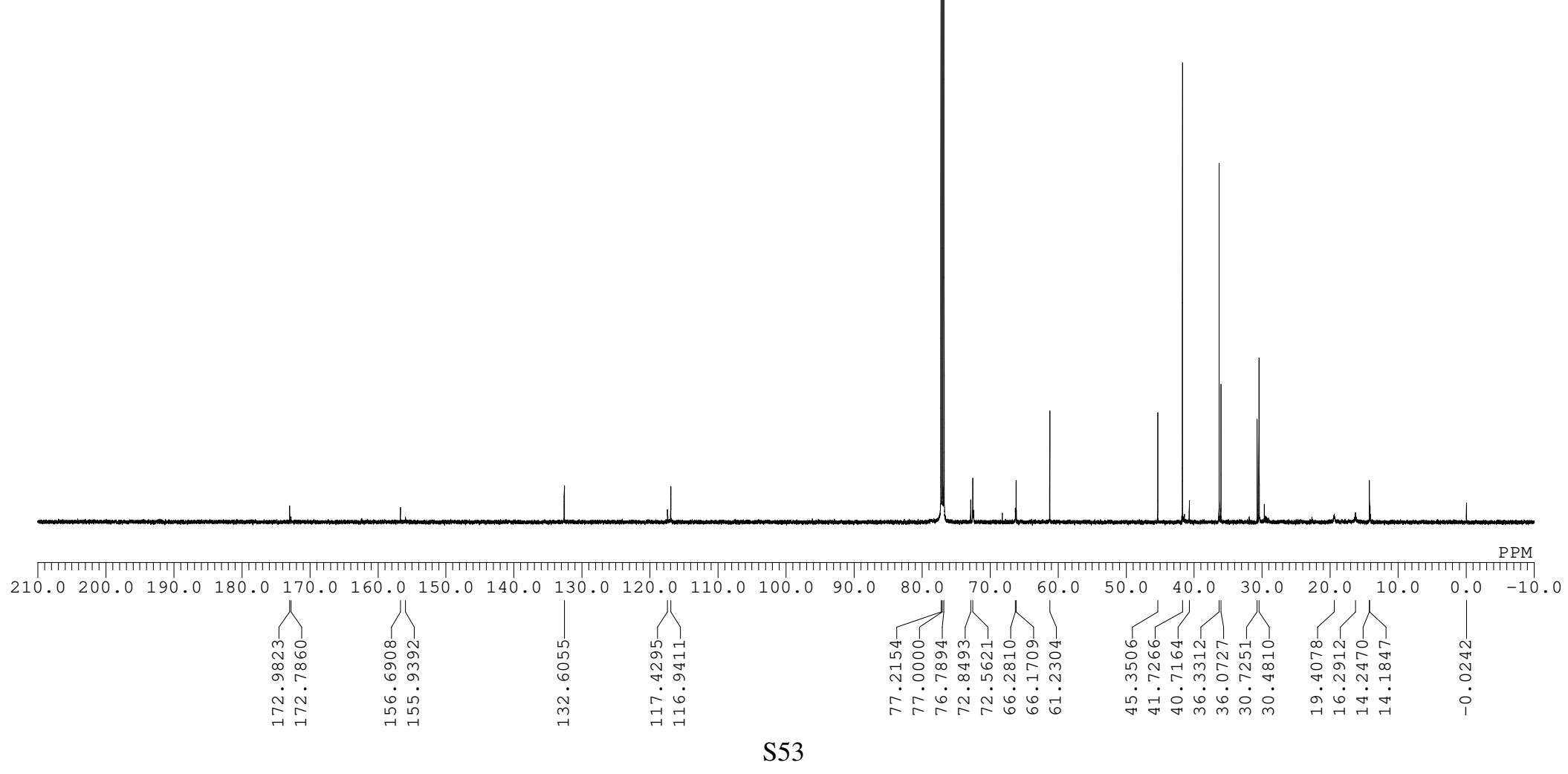




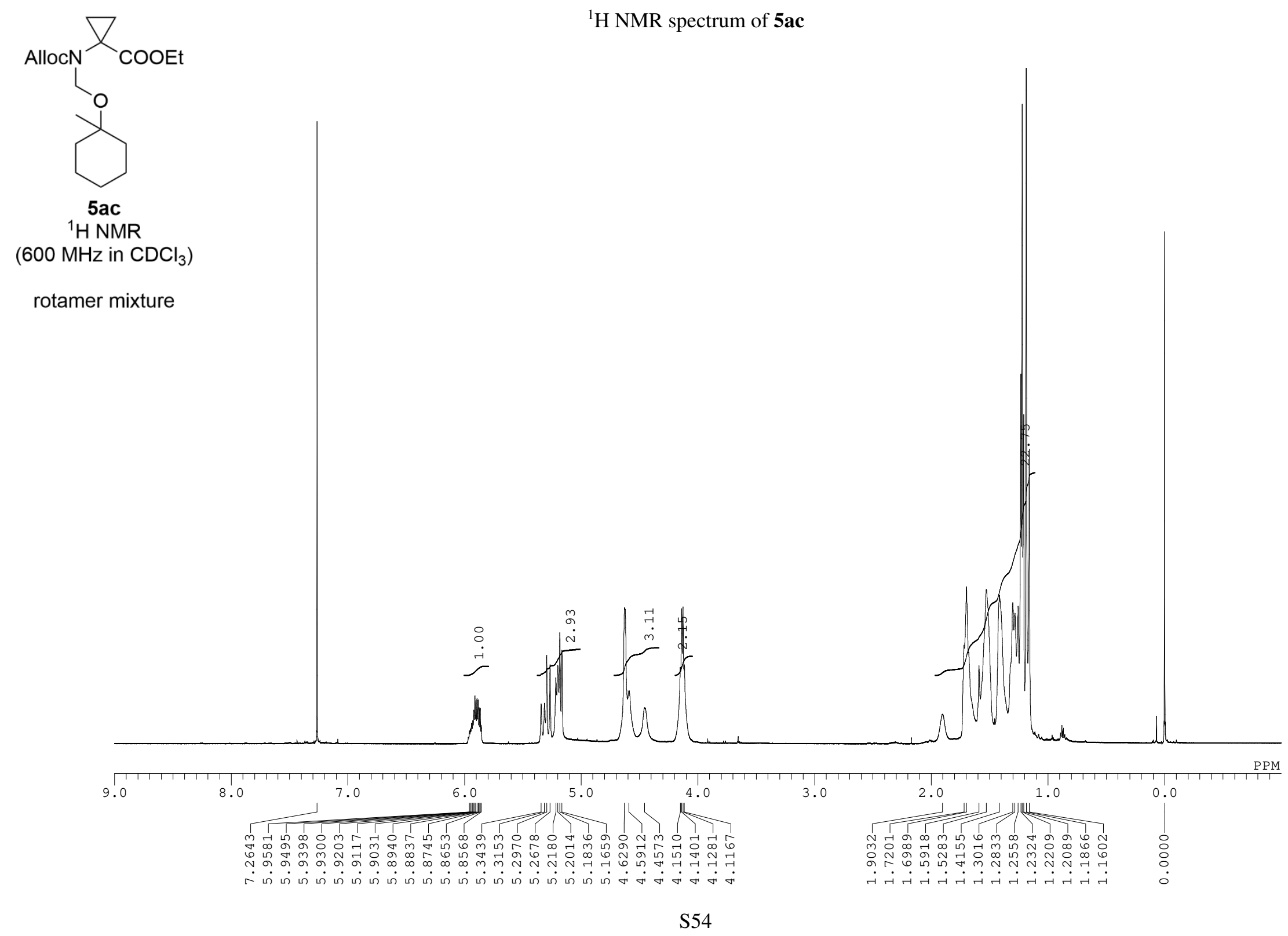




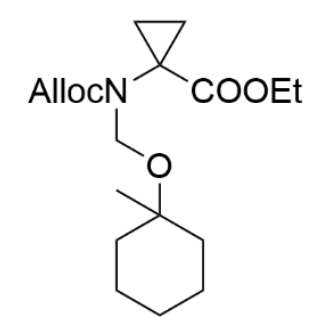

${ }^{13} \mathrm{C}\left\{{ }^{1} \mathrm{H}\right\}$ NMR spectrum of $\mathbf{5 a c}$

${ }^{13} \mathrm{C}\left\{{ }^{1} \mathrm{H}\right\}$ NMR

$\left(150 \mathrm{MHz}\right.$ in $\left.\mathrm{CDCl}_{3}\right)$

rotamer mixture

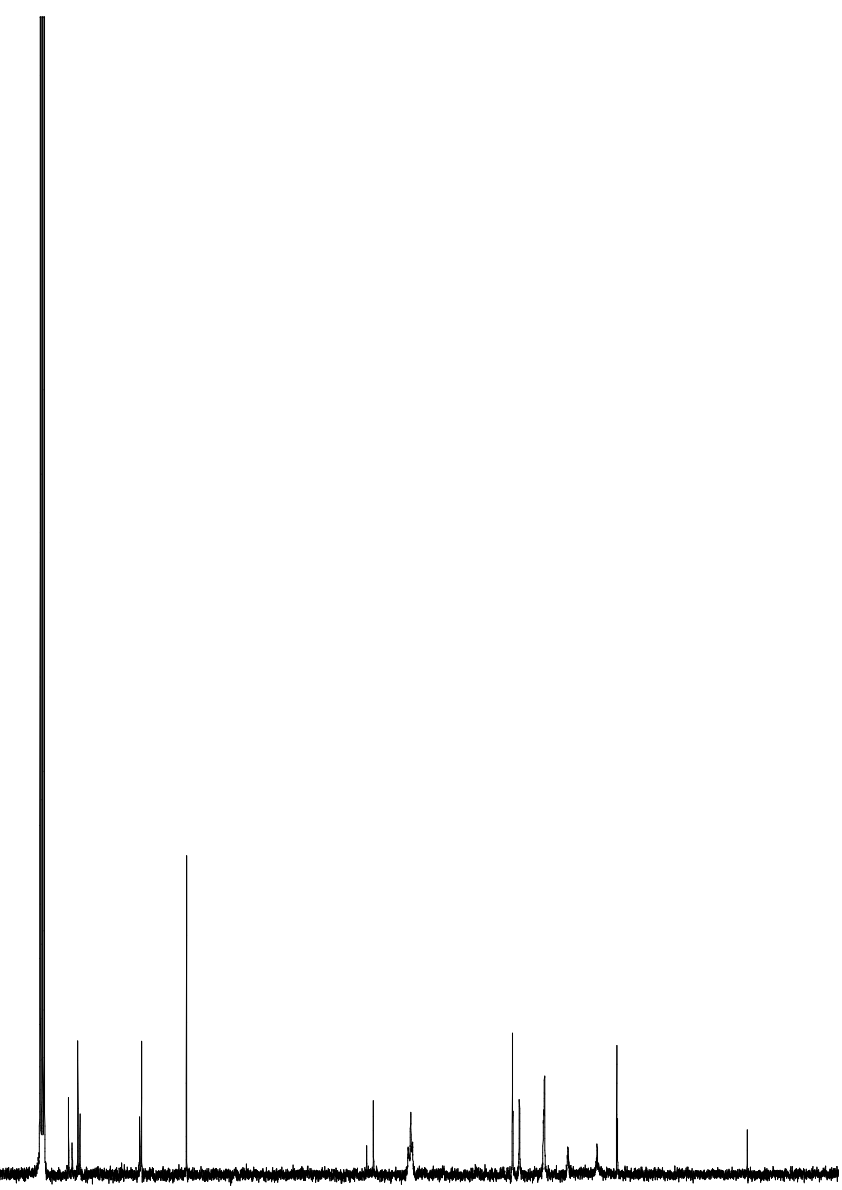

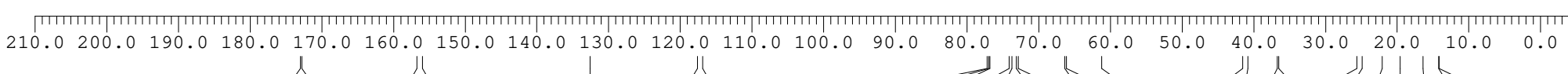
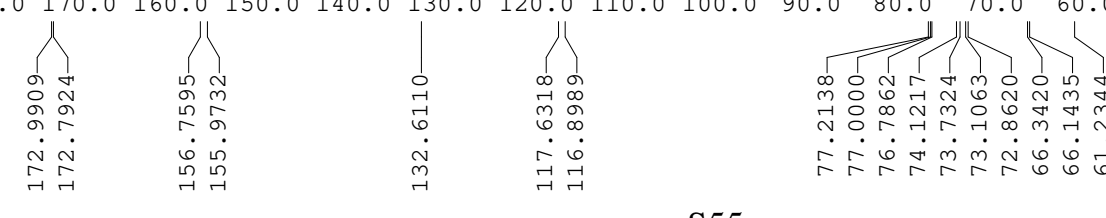


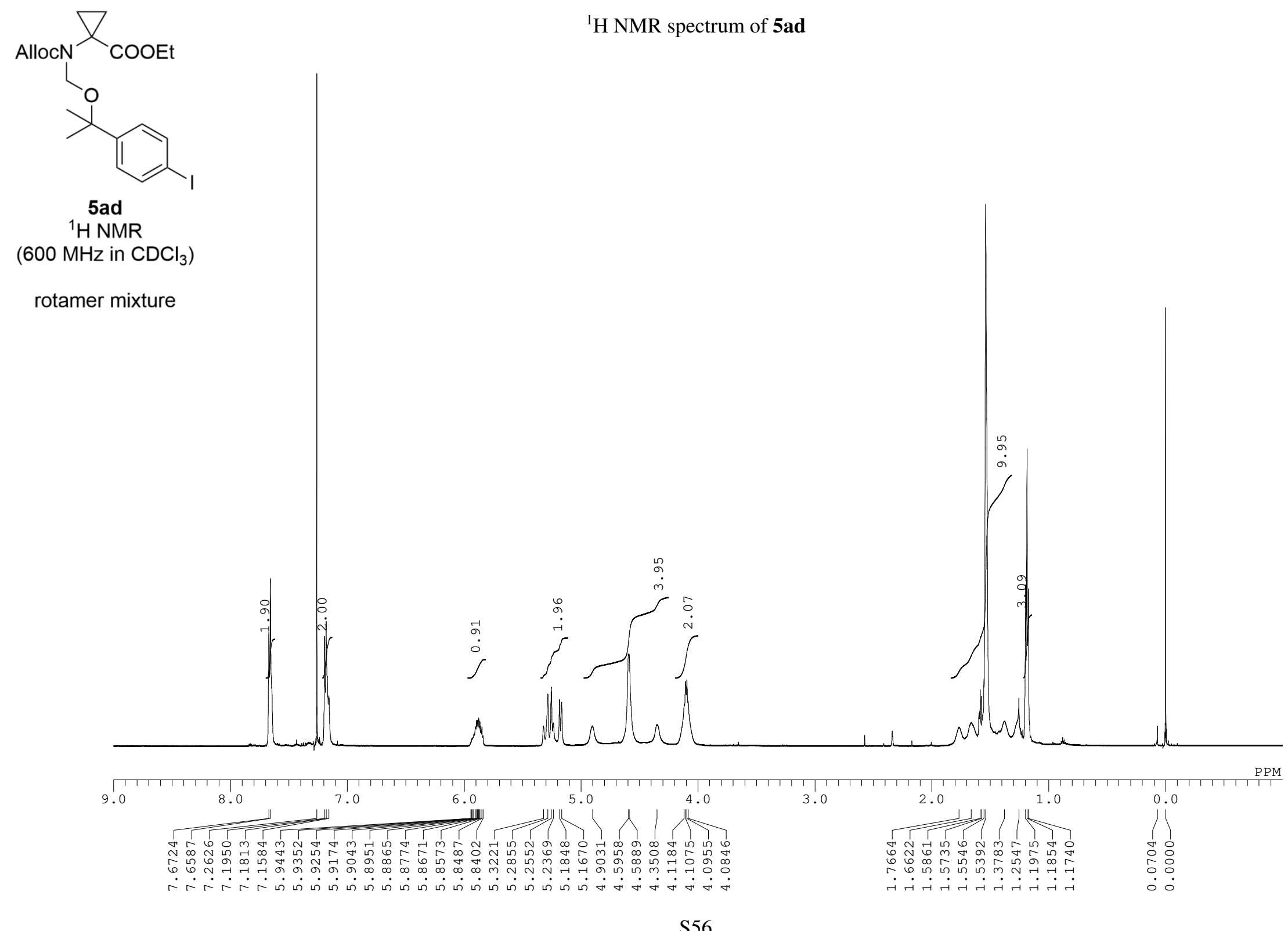




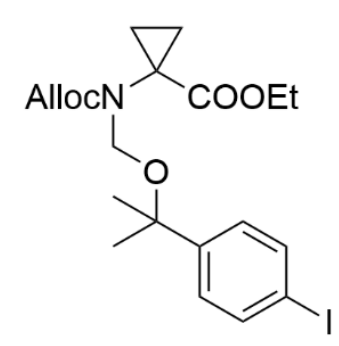

${ }^{13} \mathrm{C}\left\{{ }^{1} \mathrm{H}\right\}$ NMR spectrum of $\mathbf{5 a d}$

$5 \mathrm{ad}$

${ }^{13} \mathrm{C}\left\{{ }^{1} \mathrm{H}\right\}$ NMR

$\left(150 \mathrm{MHz}\right.$ in $\left.\mathrm{CDCl}_{3}\right)$

rotamer mixture

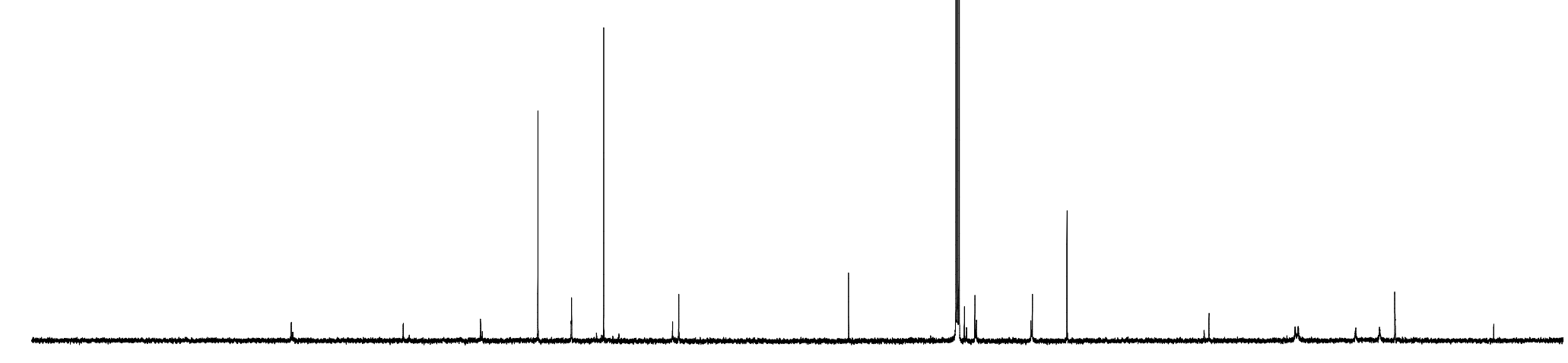

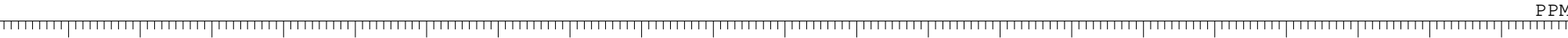

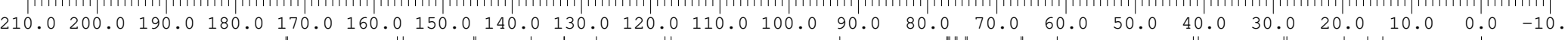
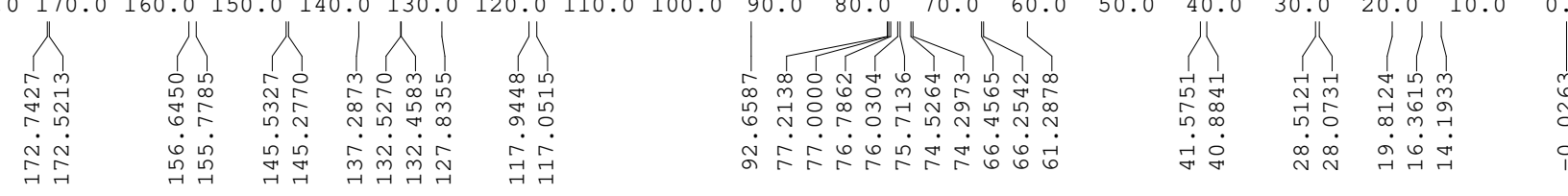


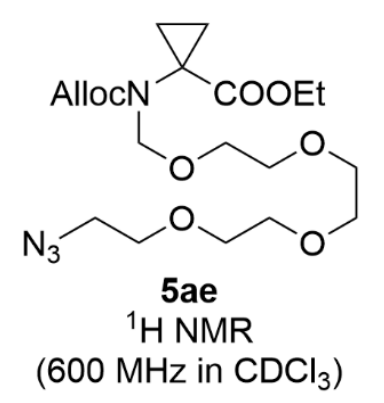

${ }^{1} \mathrm{H}$ NMR spectrum of $\mathbf{5 a e}$

rotamer mixture

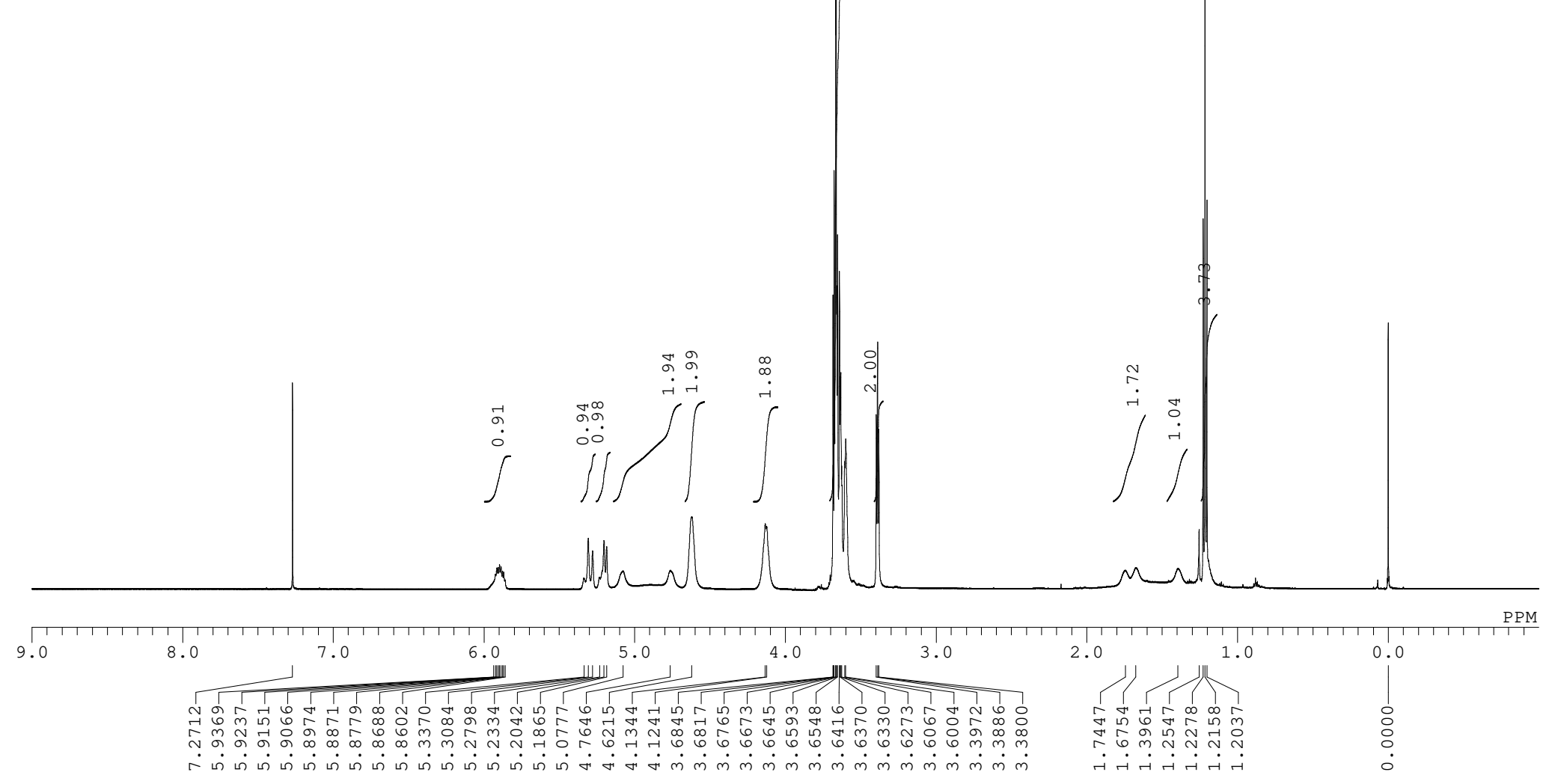




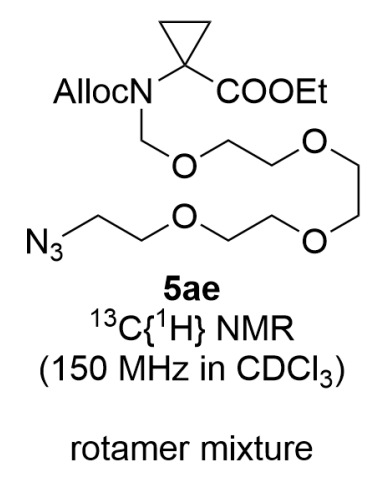

${ }^{13} \mathrm{C}\left\{{ }^{1} \mathrm{H}\right\}$ NMR spectrum of $\mathbf{5 a e}$

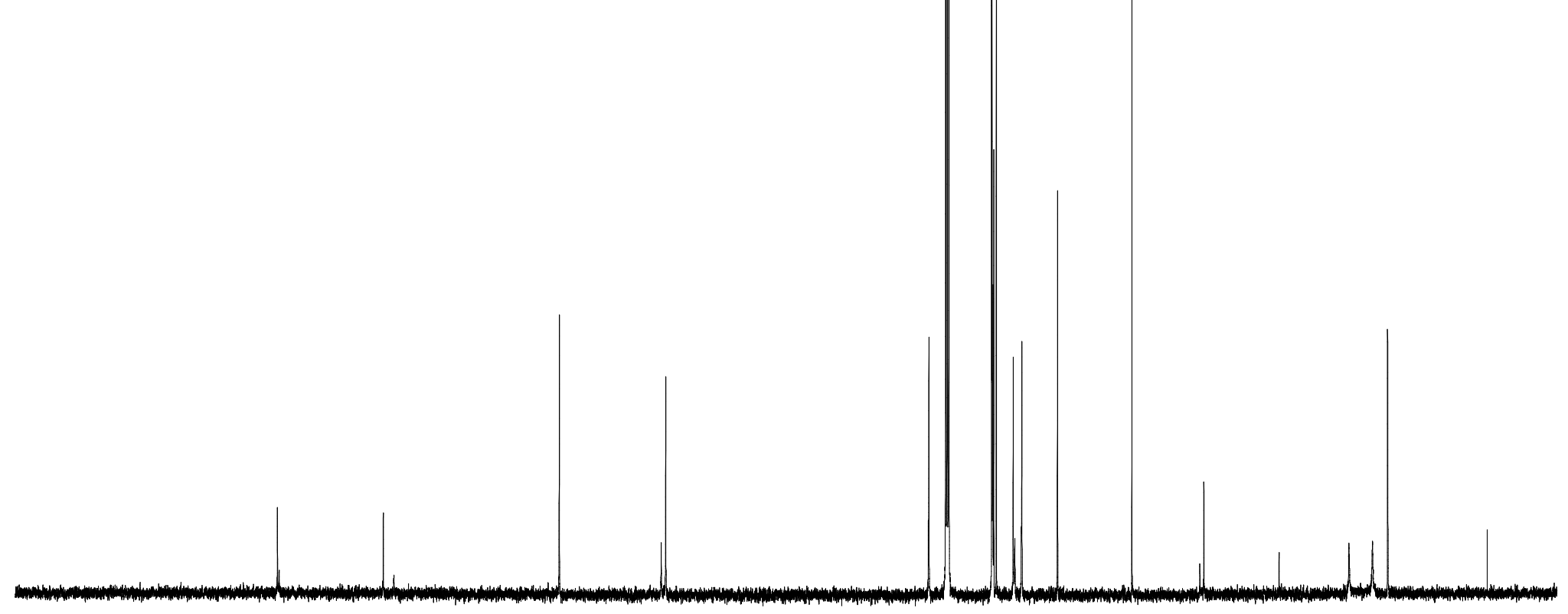

(2)

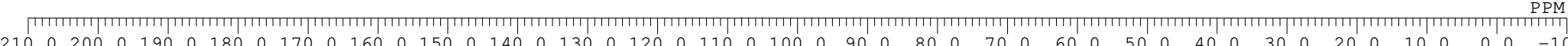

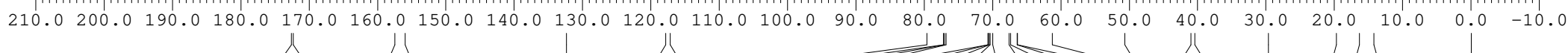

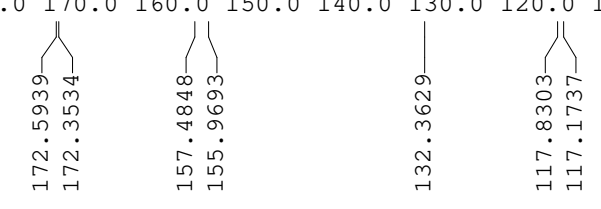

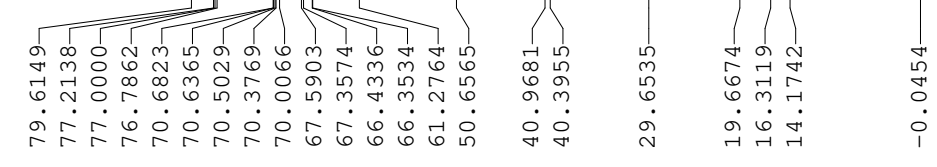




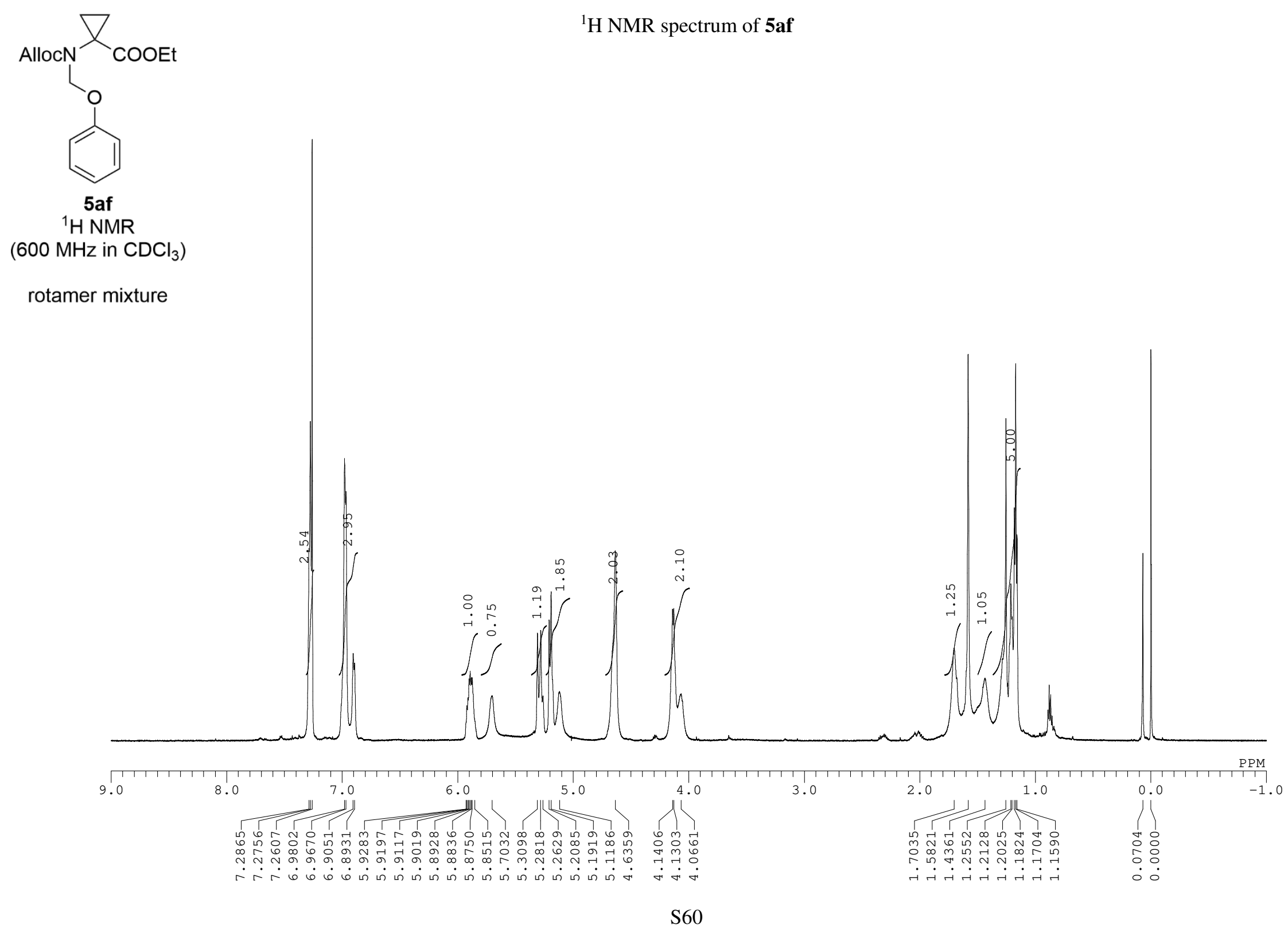




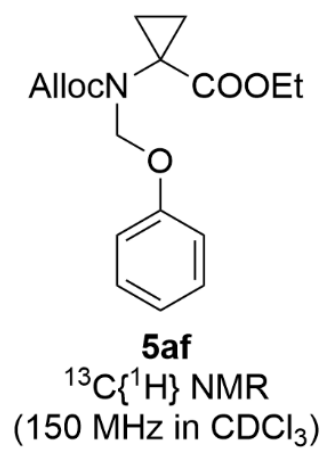

${ }^{13} \mathrm{C}\left\{{ }^{1} \mathrm{H}\right\}$ NMR spectrum of $\mathbf{5 a f}$

rotamer mixture

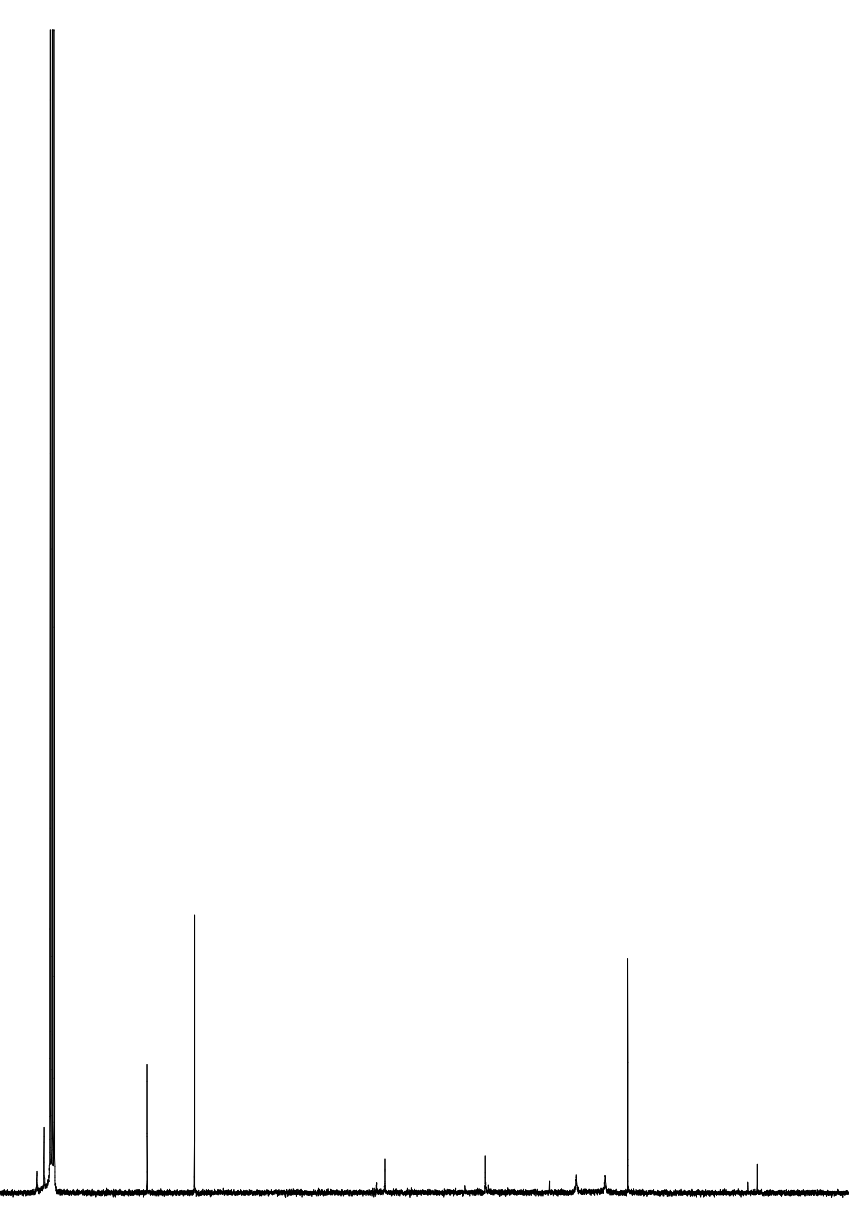

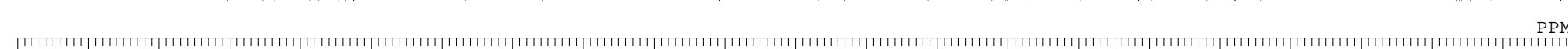

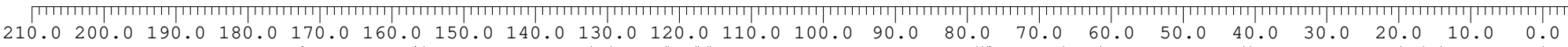
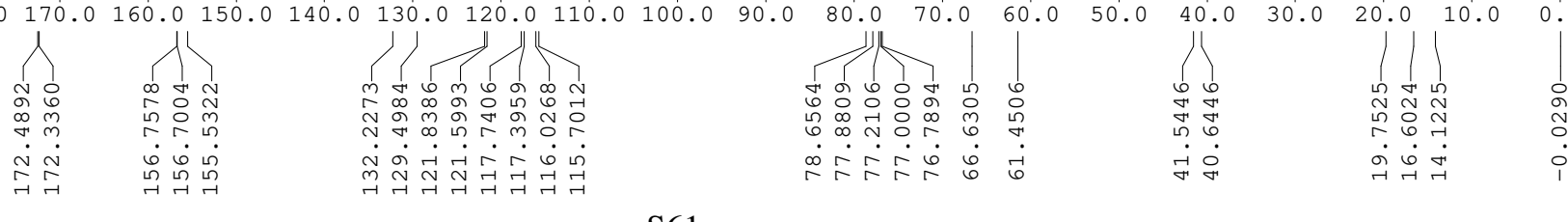


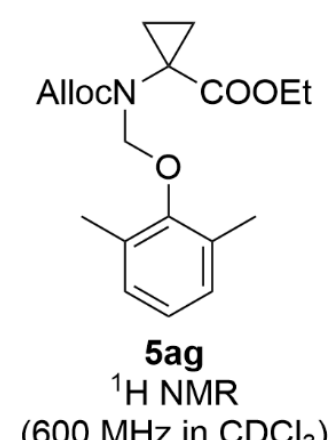

${ }^{1} \mathrm{H}$ NMR spectrum of $\mathbf{5 a g}$

(600 MHz in $\mathrm{CDCl}_{3}$

$3: 2$ rotamer mixture

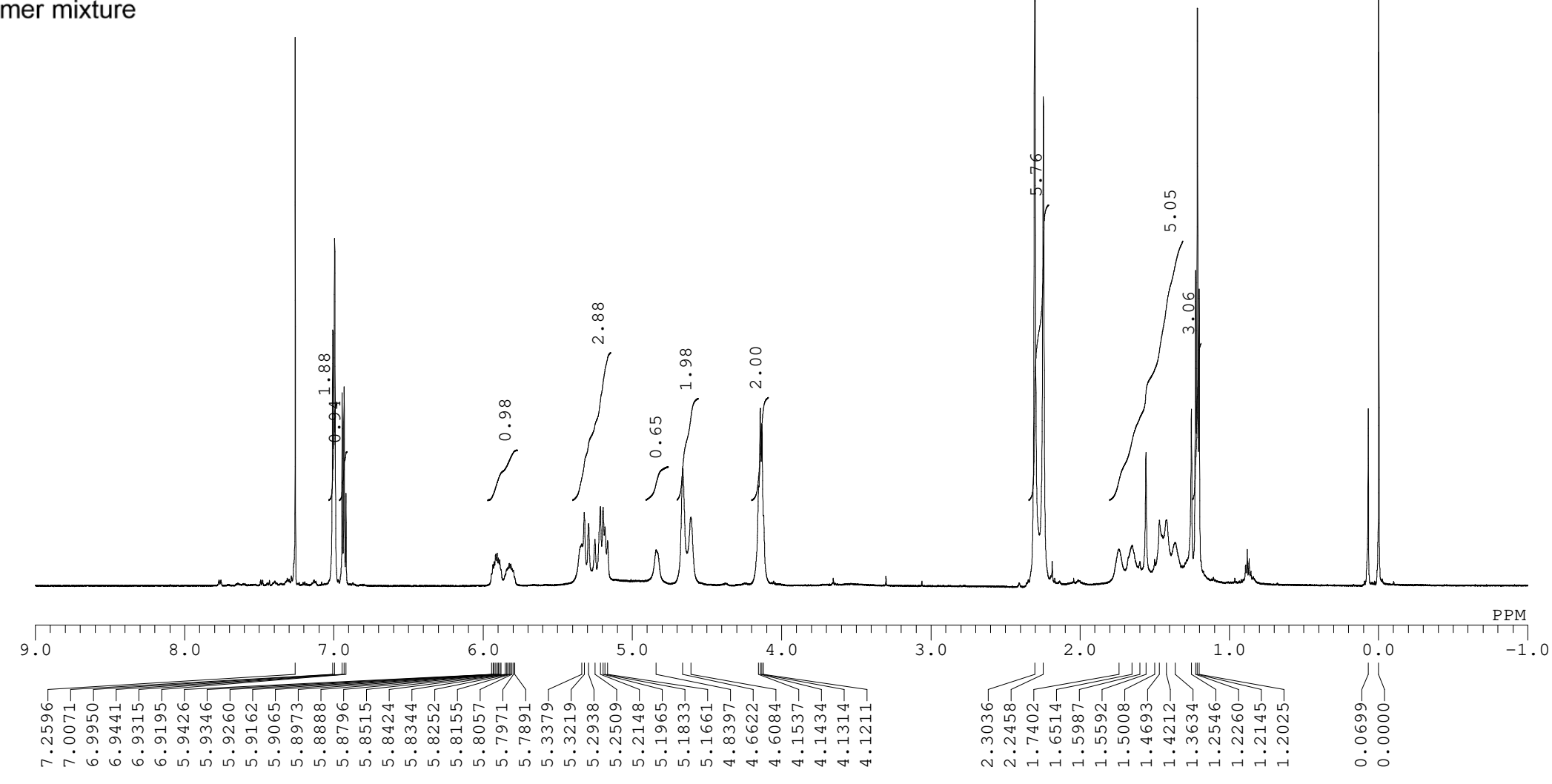




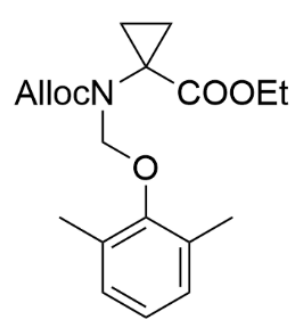

${ }^{13} \mathrm{C}\left\{{ }^{1} \mathrm{H}\right\}$ NMR spectrum of $\mathbf{5 a g}$

${ }^{13} \mathrm{C}\left\{{ }^{1} \mathrm{H}\right\} \mathrm{NM}$ $\left(150 \mathrm{MHz}\right.$ in $\mathrm{CDCl}_{3}$ )

$3: 2$ rotamer mixture

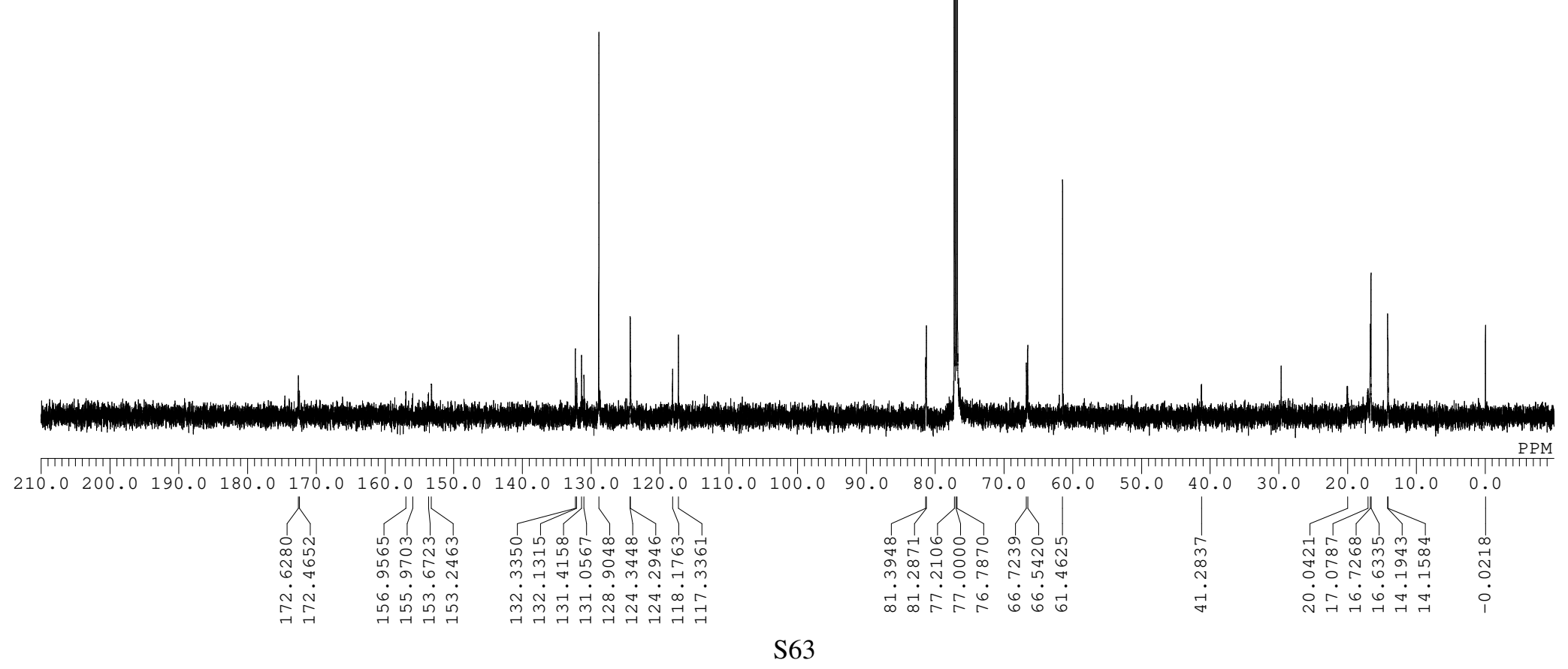




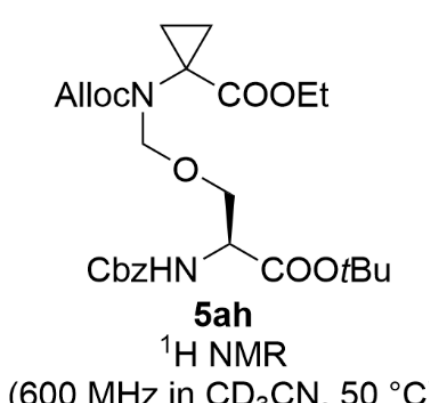

${ }^{1} \mathrm{H}$ NMR spectrum of $\mathbf{5 a h}$

$\left(600 \mathrm{MHz}\right.$ in $\left.\mathrm{CD}_{3} \mathrm{CN}, 50^{\circ} \mathrm{C}\right)$

rotamer mixture

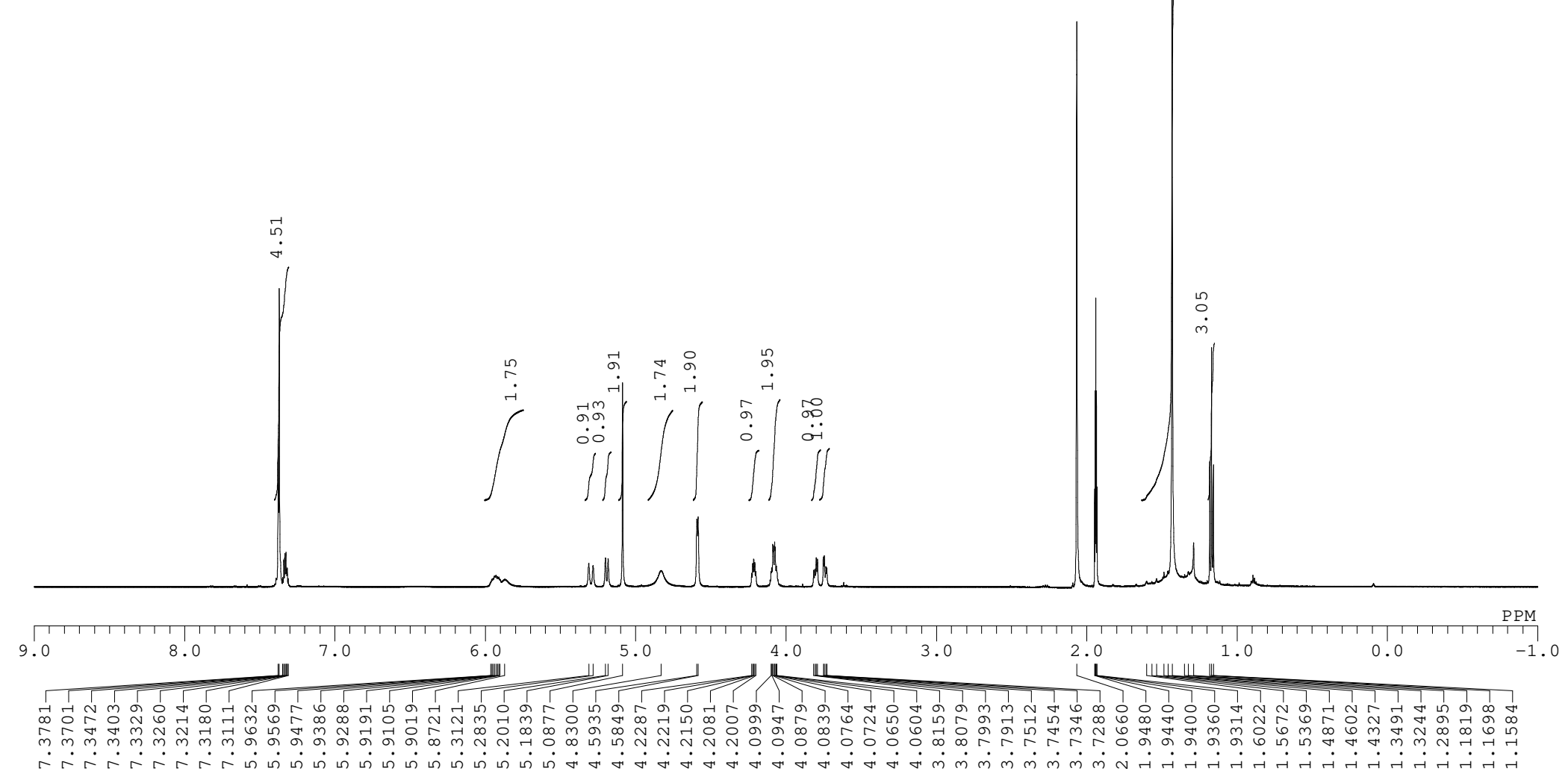




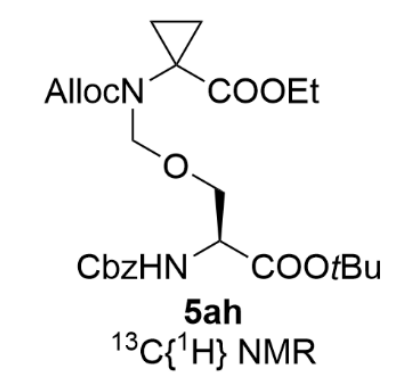

${ }^{13} \mathrm{C}\left\{{ }^{1} \mathrm{H}\right\}$ NMR spectrum of $\mathbf{5 a h}$

(150 $\mathrm{MHz}$ in $\mathrm{CD}_{3} \mathrm{CN}, 50^{\circ} \mathrm{C}$ )

rotamer mixture

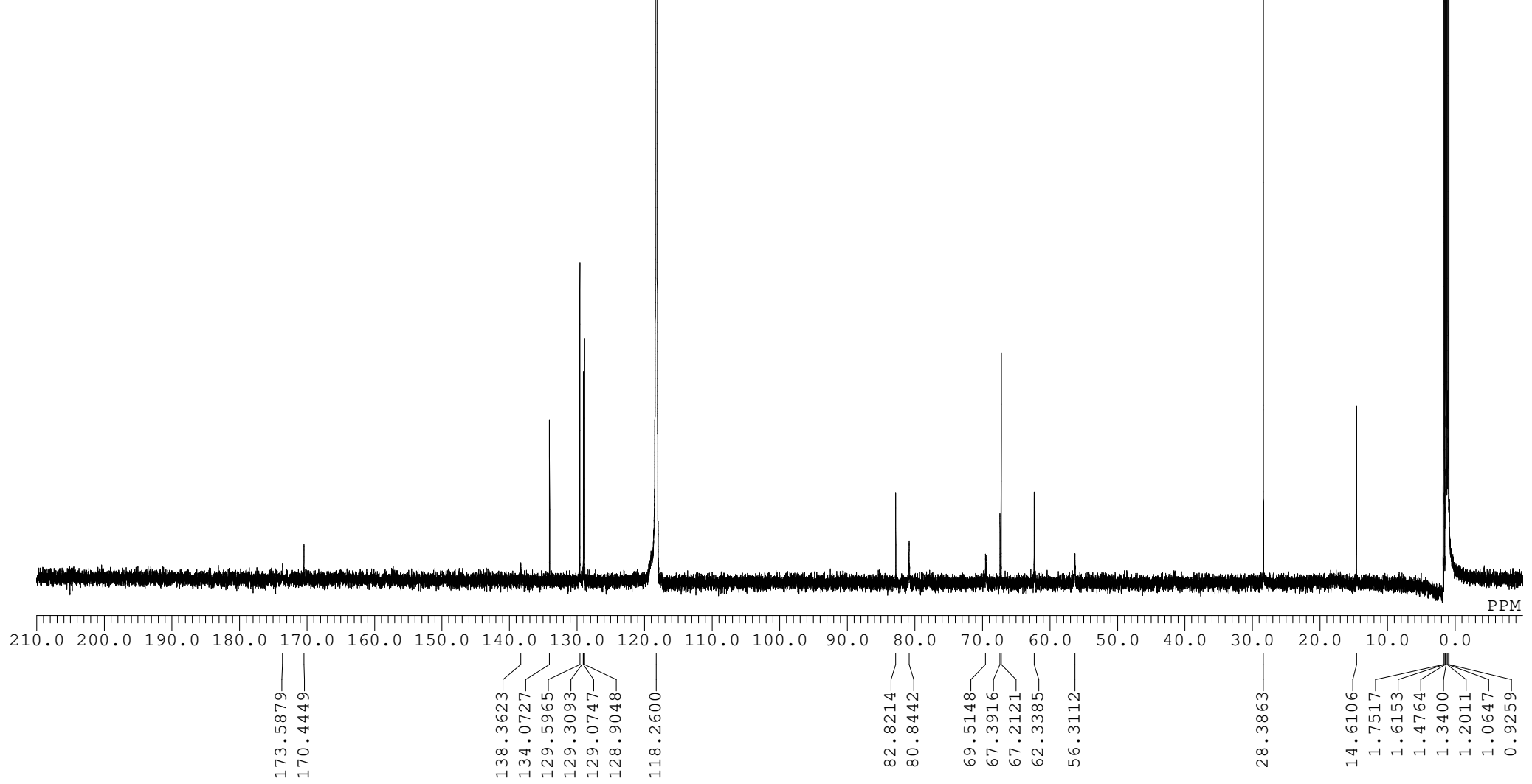




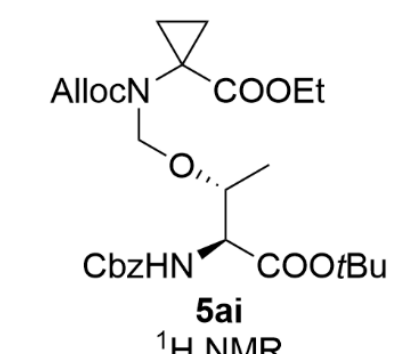

${ }^{1}$ H NMR spectrum of $\mathbf{5 a i}$

$\left(600 \mathrm{MHz}\right.$ in $\left.\mathrm{CD}_{3} \mathrm{CN}, 50^{\circ} \mathrm{C}\right)$

rotamer mixture

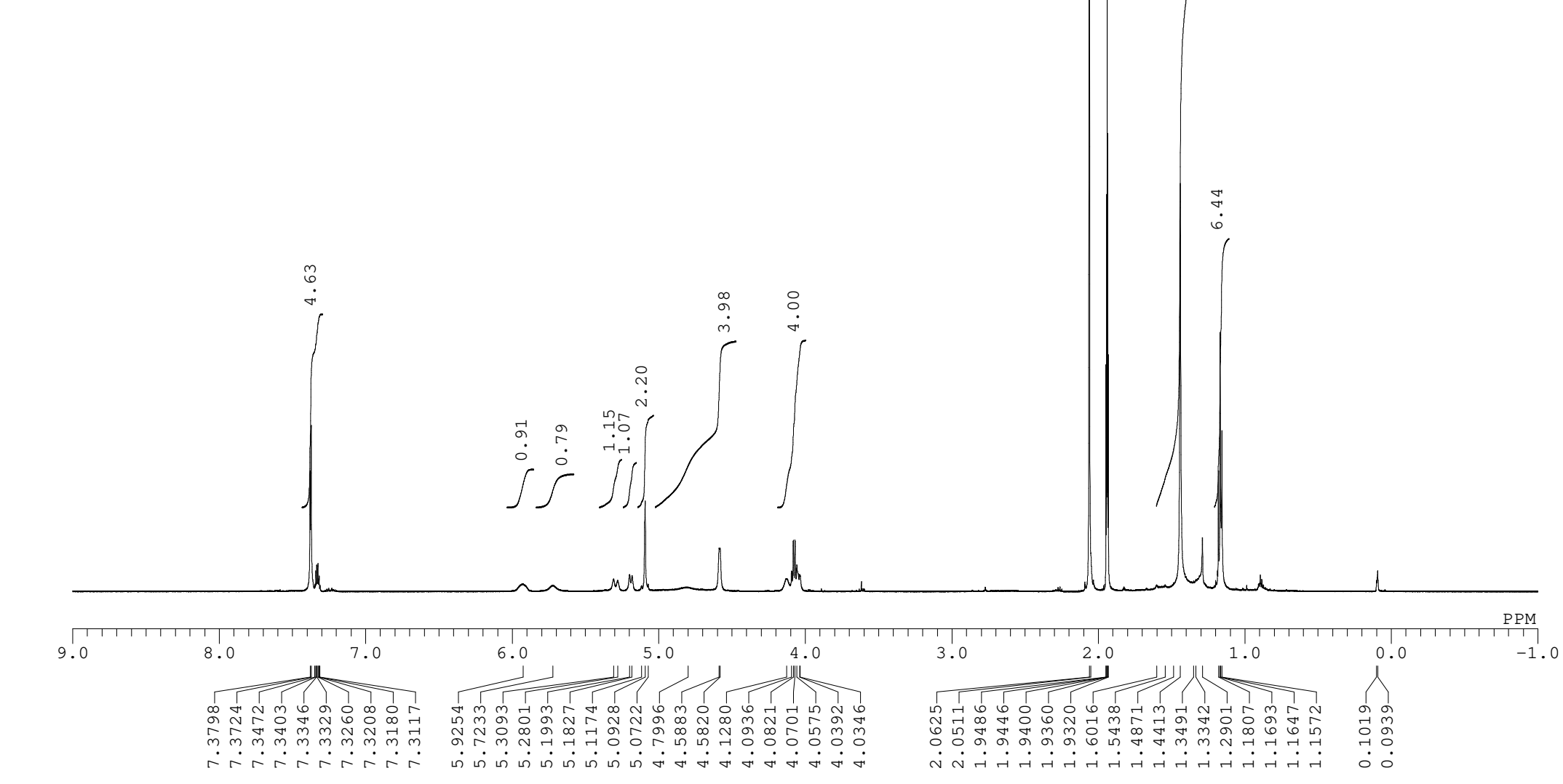




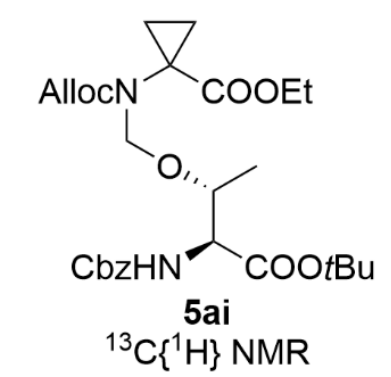

${ }^{13} \mathrm{C}\left\{{ }^{1} \mathrm{H}\right\}$ NMR spectrum of 5ai

$\left(150 \mathrm{MHz}\right.$ in $\left.\mathrm{CD}_{3} \mathrm{CN}, 50^{\circ} \mathrm{C}\right)$

rotamer mixture

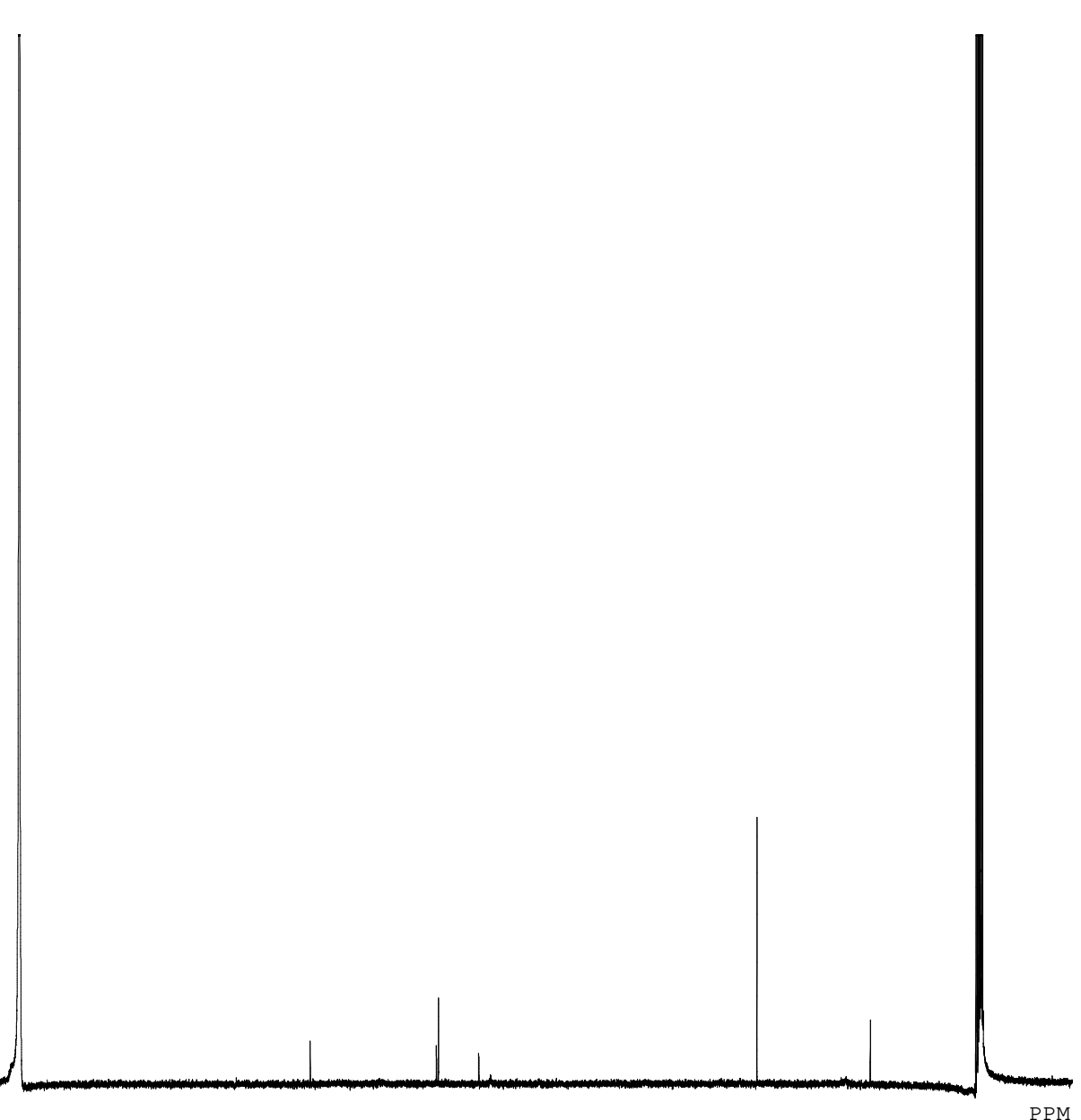

(1) PP

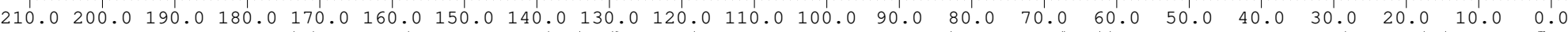

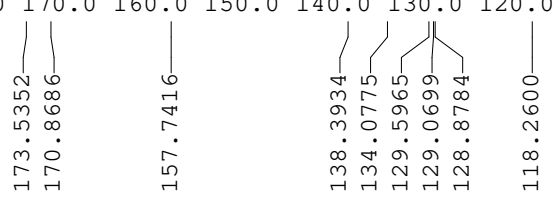
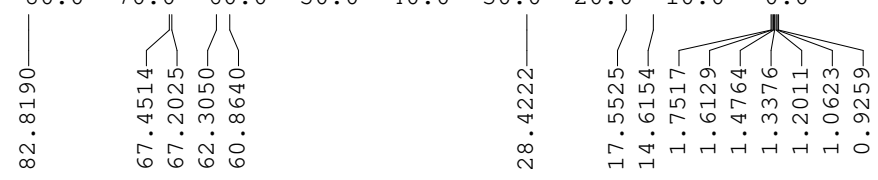


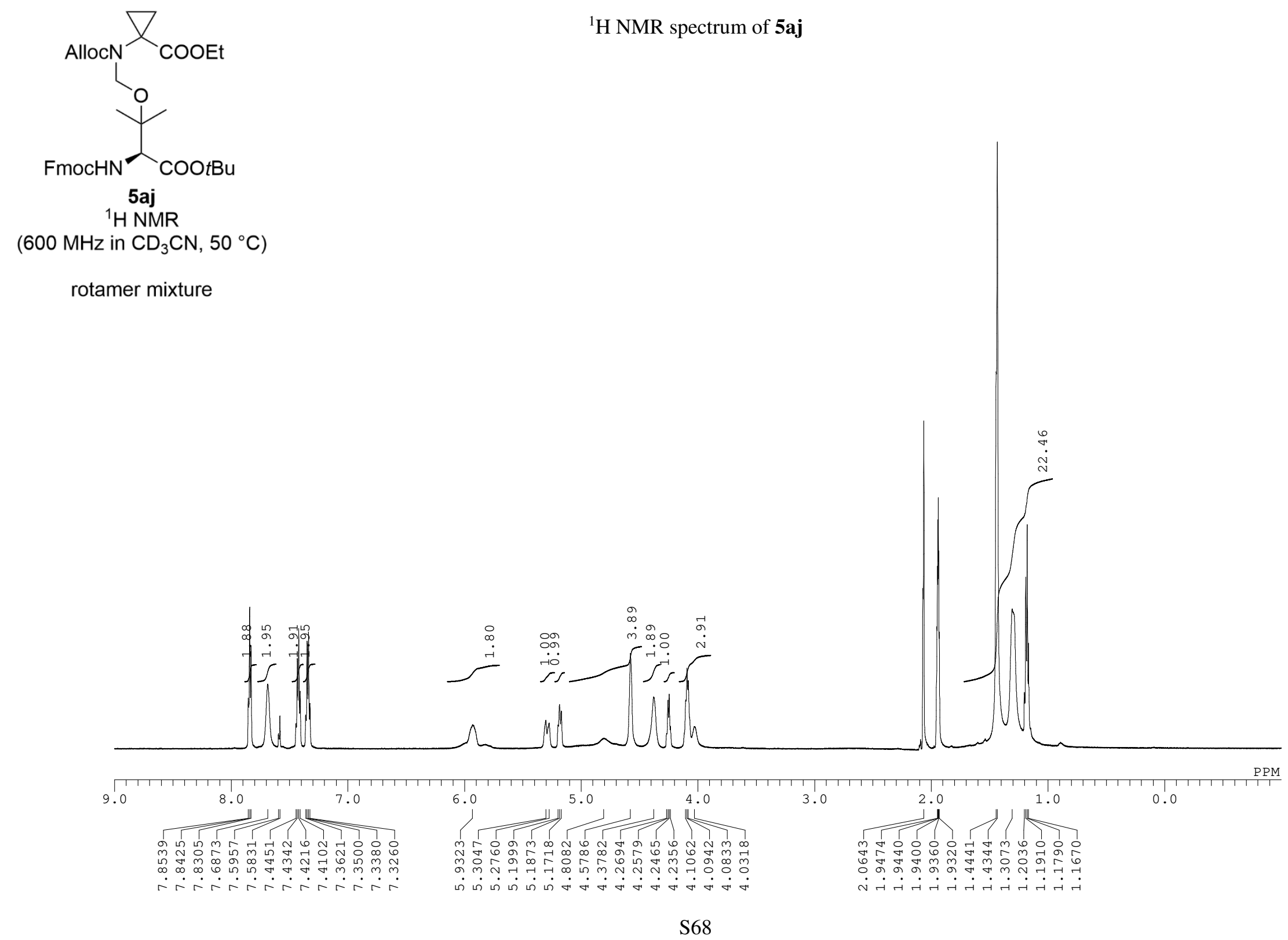




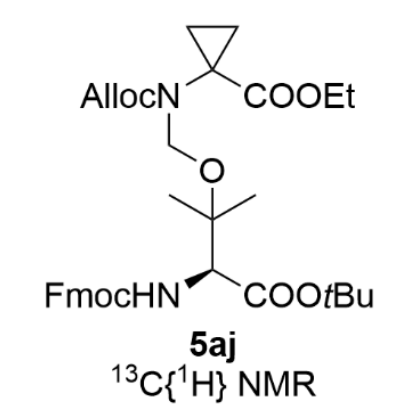

${ }^{13} \mathrm{C}\left\{{ }^{1} \mathrm{H}\right\}$ NMR spectrum of 5aj

$\left(150 \mathrm{MHz}\right.$ in $\left.\mathrm{CD}_{3} \mathrm{CN}, 50^{\circ} \mathrm{C}\right)$

rotamer mixture

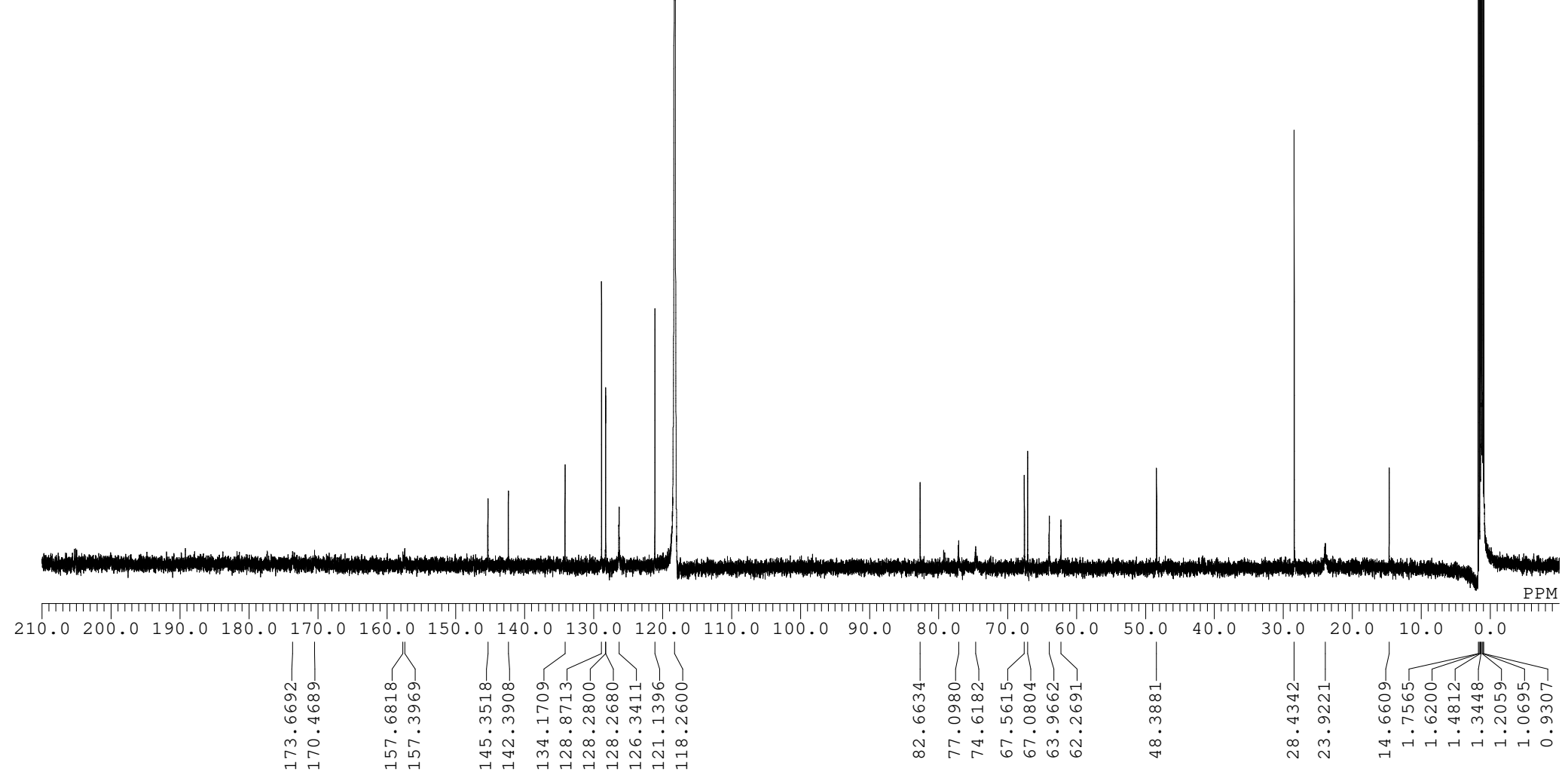




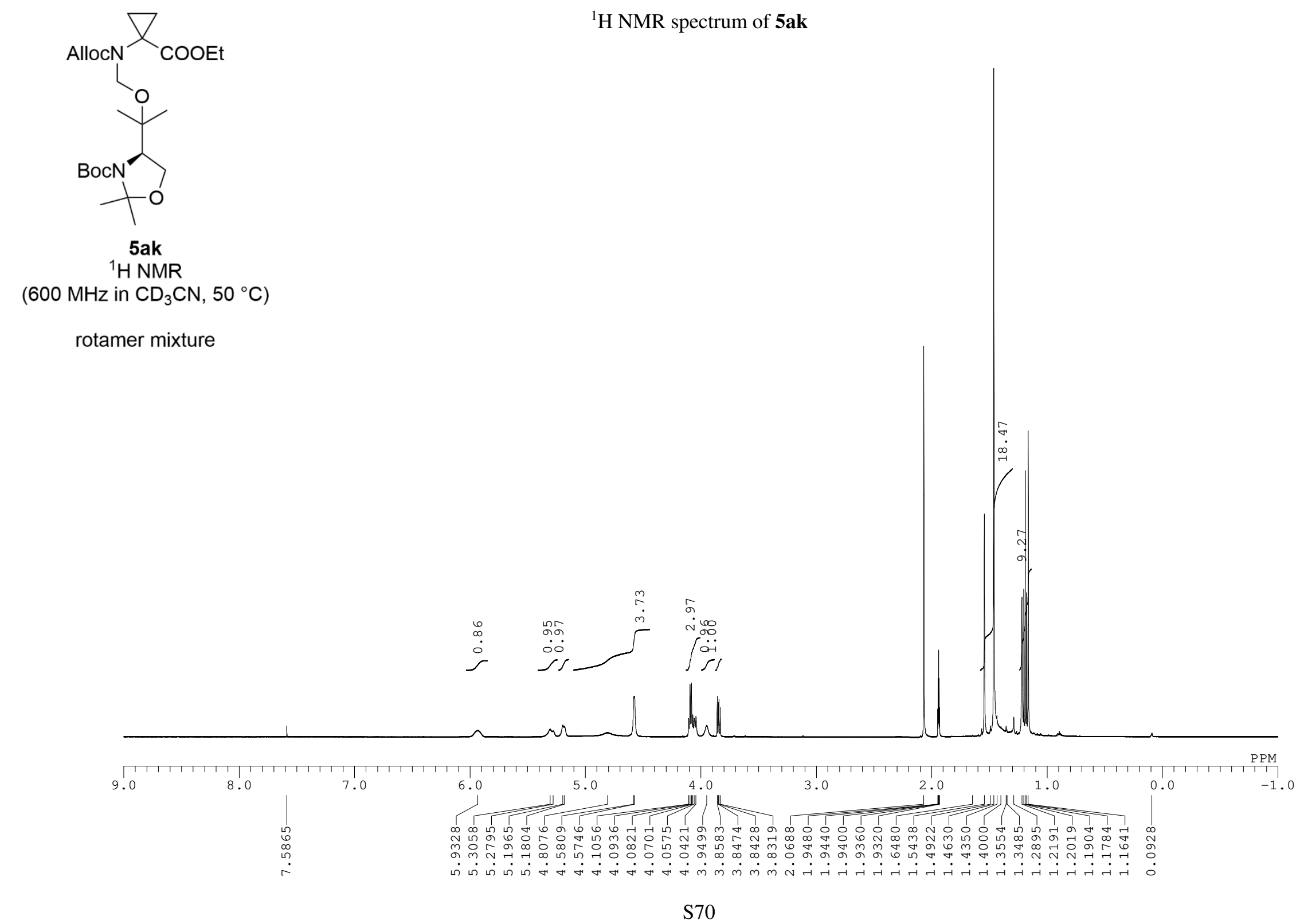




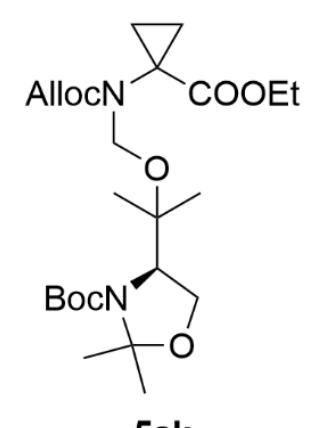

${ }^{13} \mathrm{C}\left\{{ }^{1} \mathrm{H}\right\}$ NMR spectrum of 5ak

${ }^{13} \mathrm{C}\left\{{ }^{1} \mathrm{H}\right\}$ NMR

(150 $\mathrm{MHz}$ in $\mathrm{CD}_{3} \mathrm{CN}, 50^{\circ} \mathrm{C}$ )

rotamer mixture

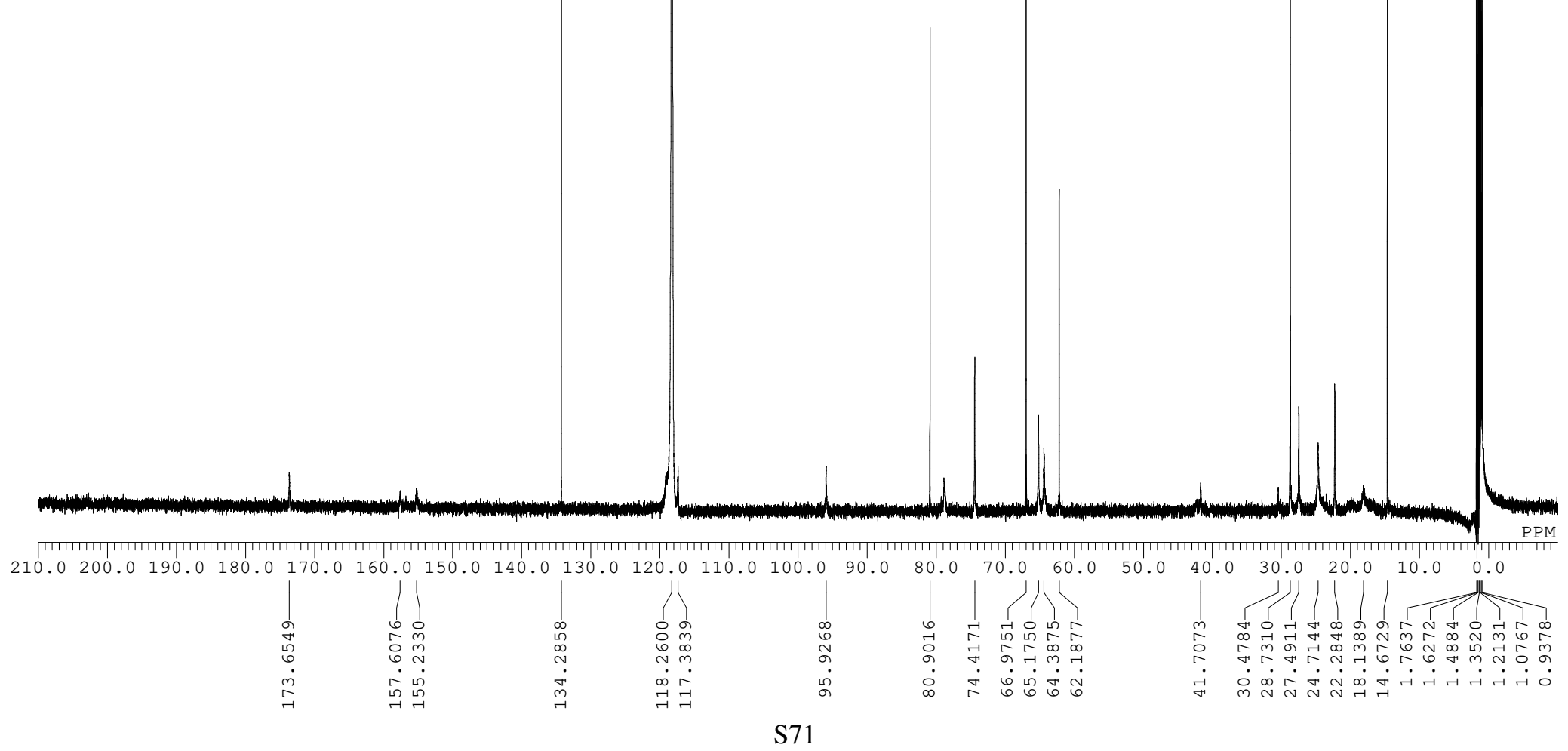




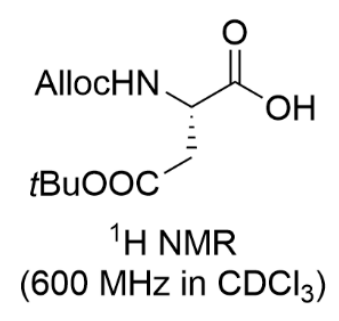

${ }^{1} \mathrm{H}$ NMR spectrum of Alloc-Asp $(t \mathrm{Bu})-\mathrm{OH}$

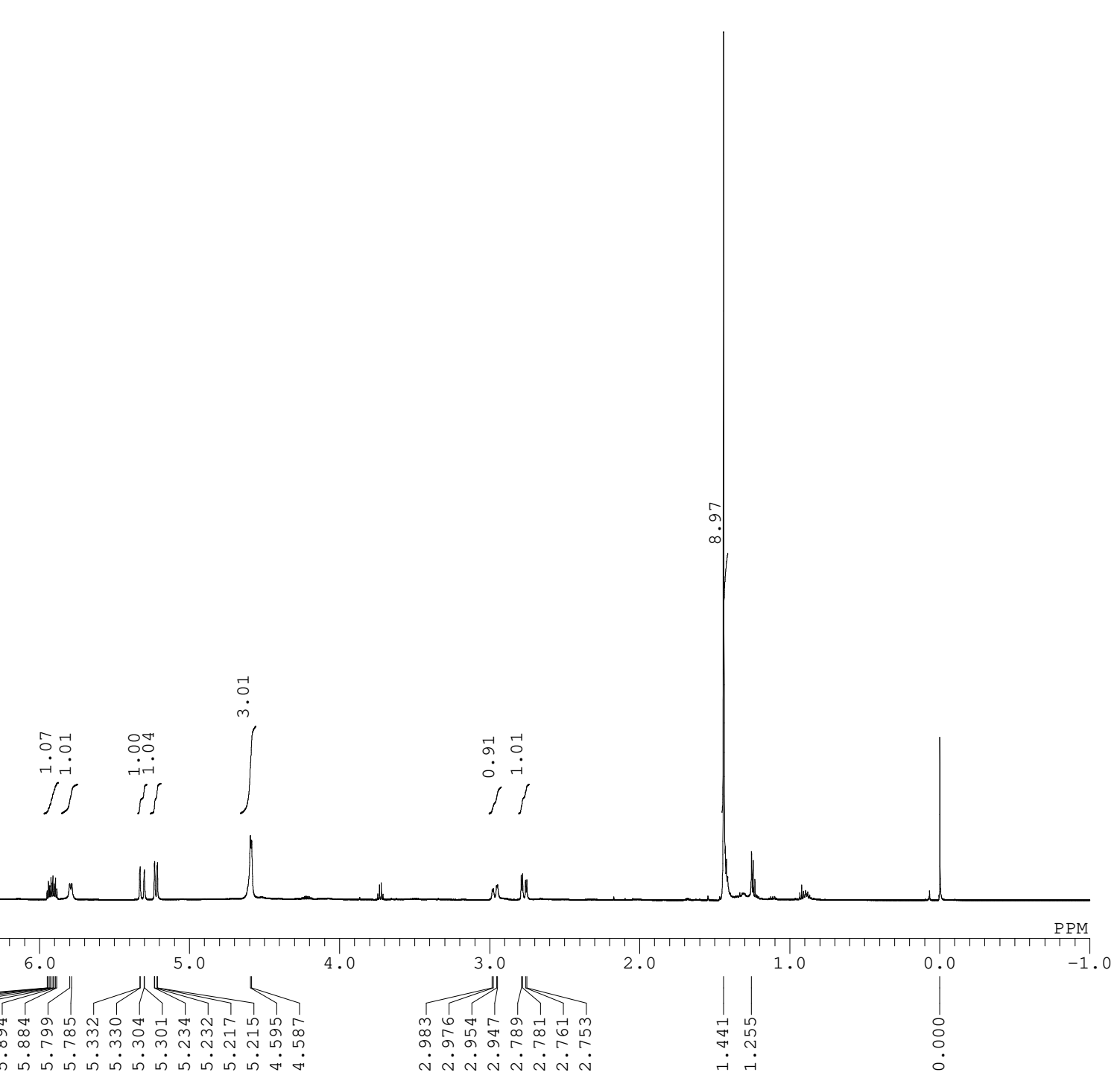




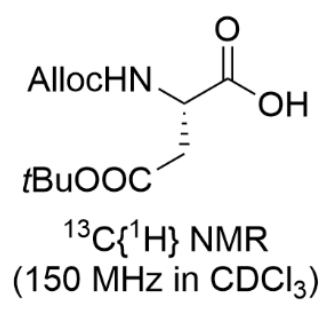

${ }^{13} \mathrm{C}\left\{{ }^{1} \mathrm{H}\right\}$ NMR spectrum of Alloc-Asp $(t \mathrm{Bu})-\mathrm{OH}$

(150 $\mathrm{MHz}$ in $\mathrm{CDCl}_{3}$ ) 


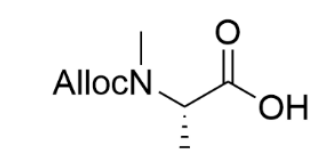

${ }^{1} \mathrm{H}$ NMR spectrum of Alloc-MeAsp $(t \mathrm{Bu})-\mathrm{OH}$

tBuOOC

${ }^{1} \mathrm{H}$ NMR

(400 $\mathrm{MHz}$ in $\mathrm{CDCl}_{3}$ )

rotamer mixture

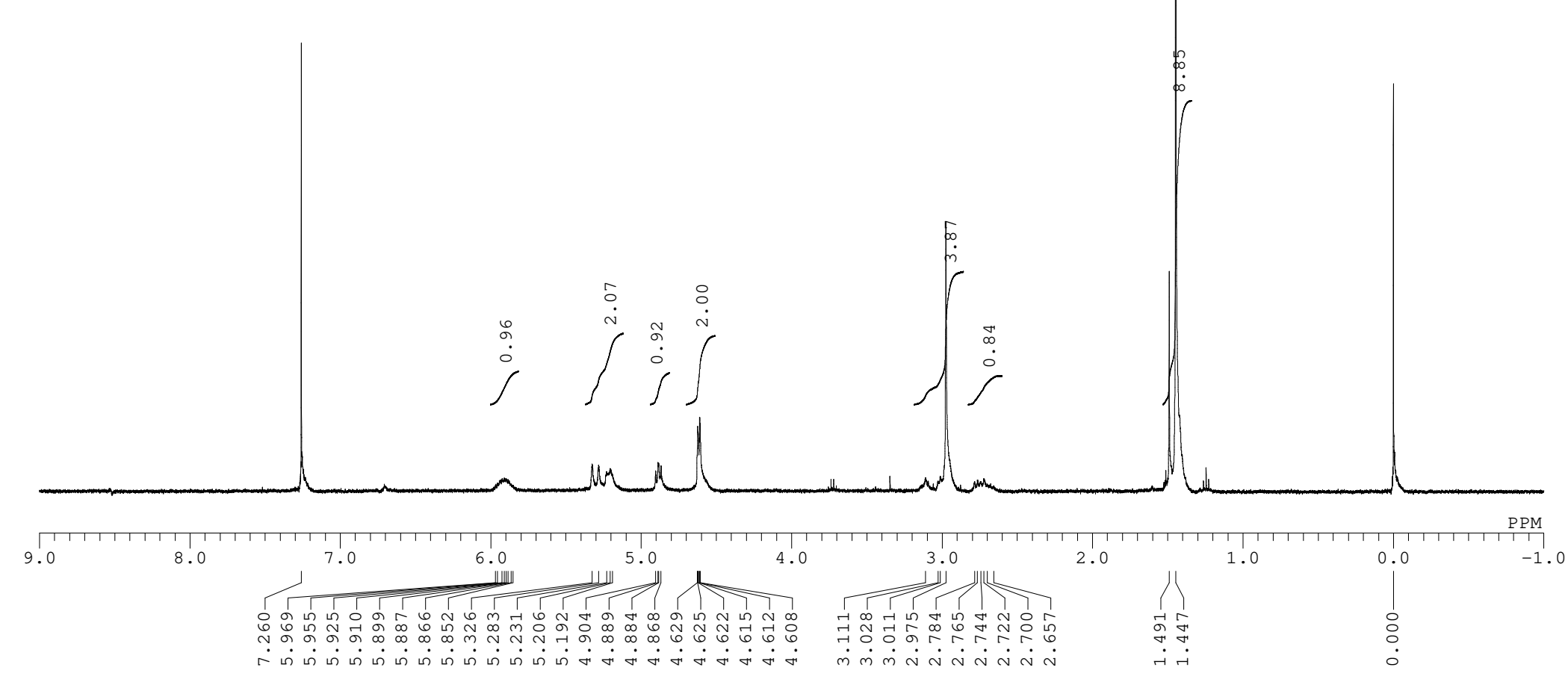




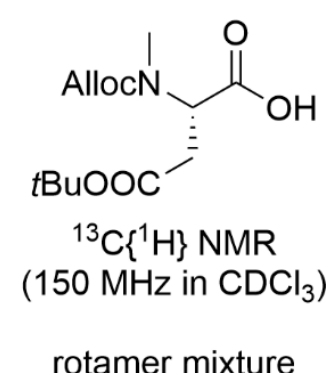

${ }^{13} \mathrm{C}\left\{{ }^{1} \mathrm{H}\right\}$ NMR spectrum of Alloc-MeAsp $(t \mathrm{Bu})-\mathrm{OH}$

rotamer mixture

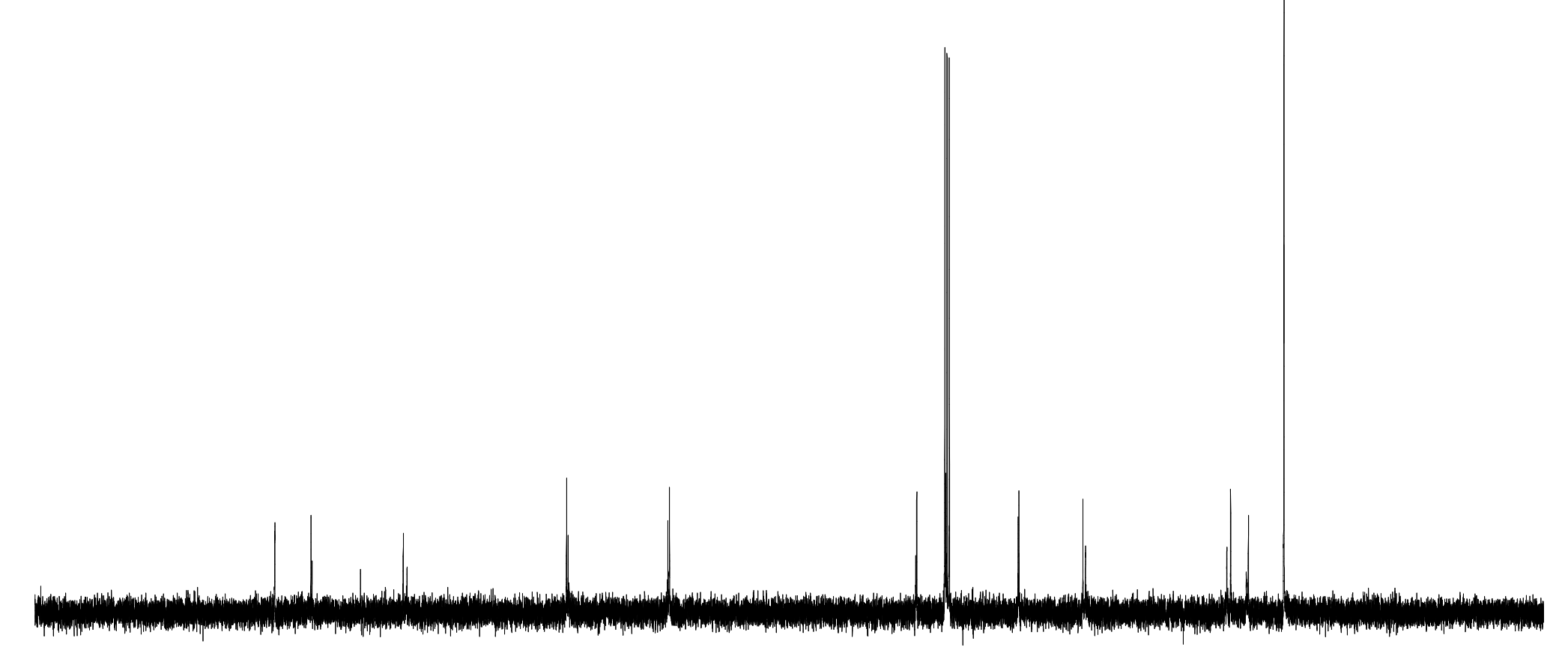

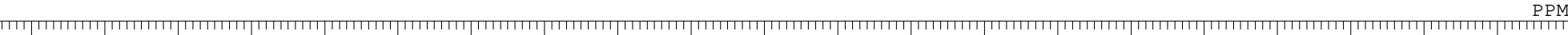

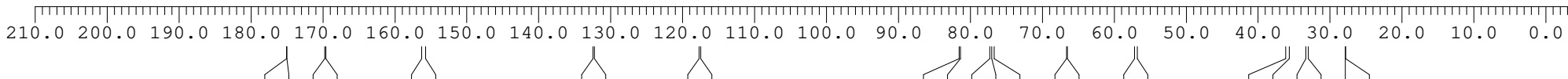

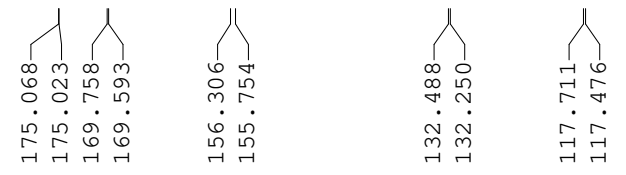

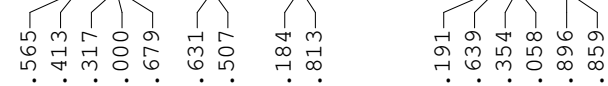

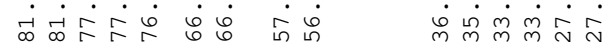




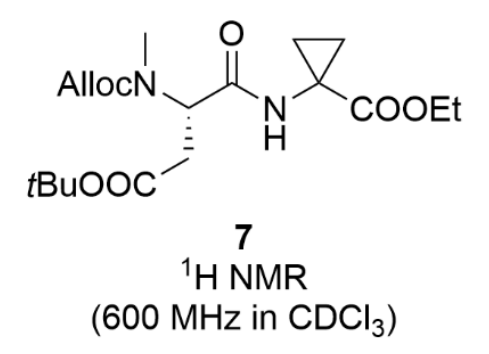

2:1 rotamer mixture
${ }^{1} \mathrm{H}$ NMR spectrum of 7

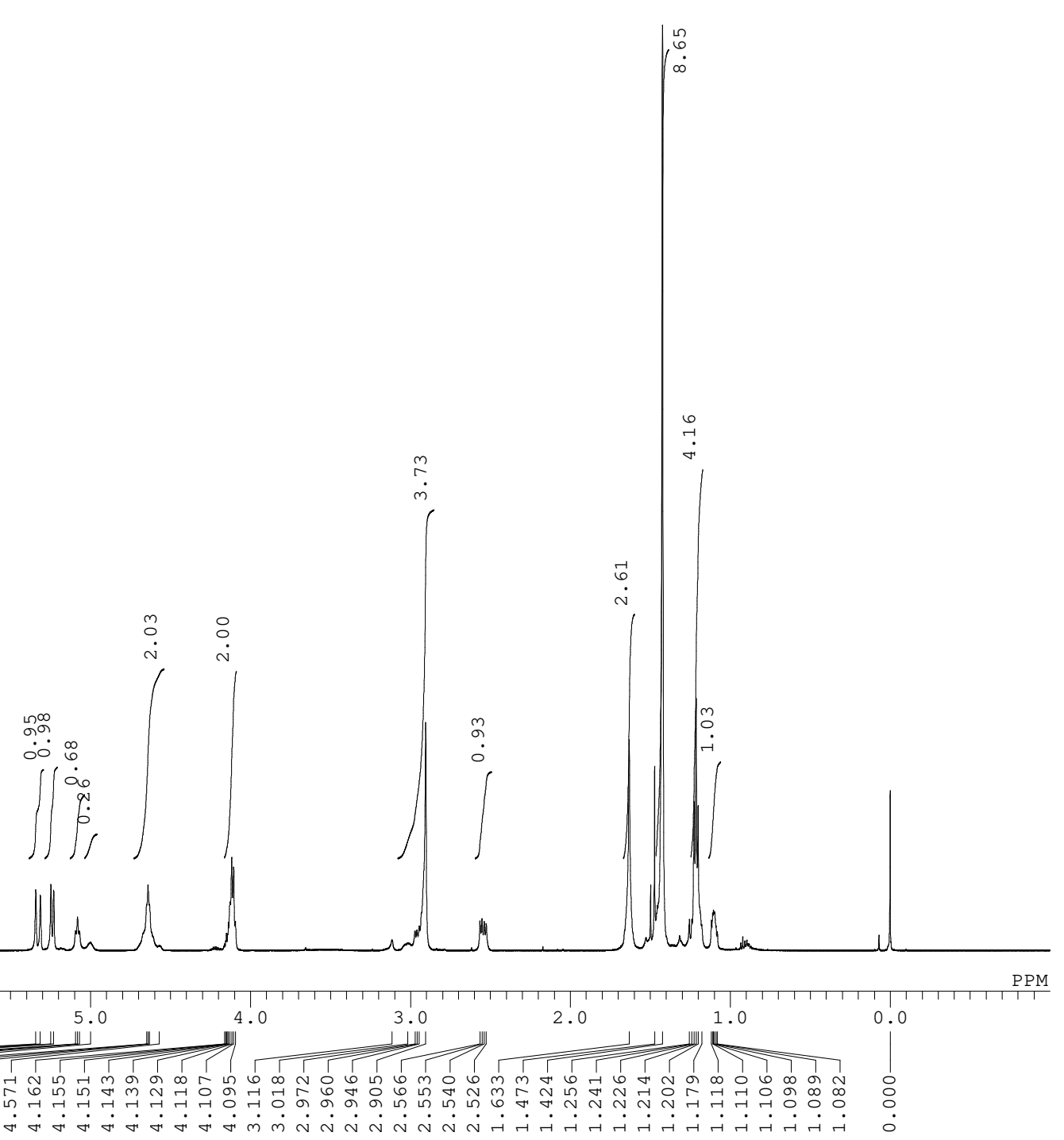




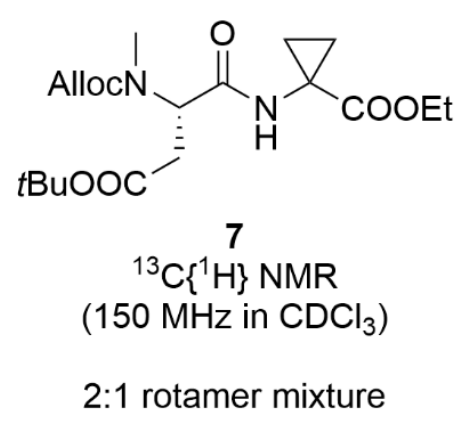

${ }^{13} \mathrm{C}\left\{{ }^{1} \mathrm{H}\right\}$ NMR spectrum of 7

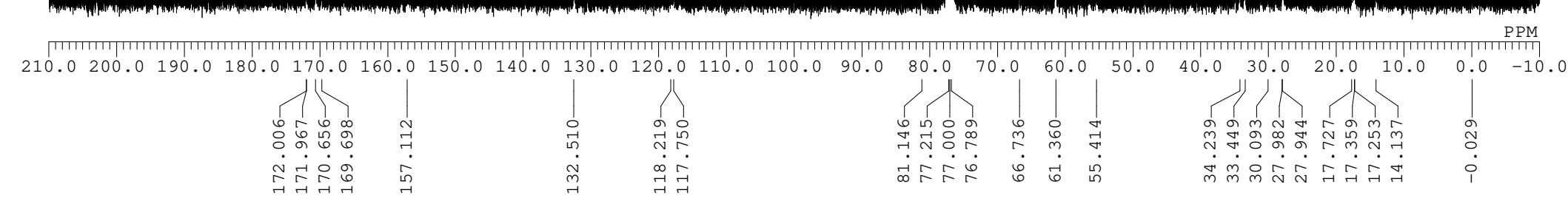

S77 


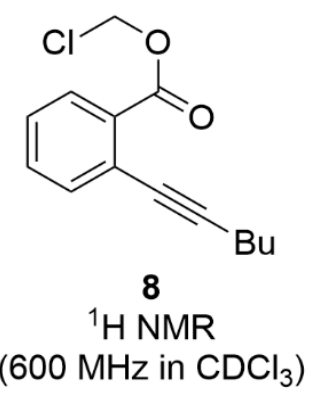

${ }^{1} \mathrm{H}$ NMR spectrum of 8

(600 MHz in $\mathrm{CDCl}_{3}$ )

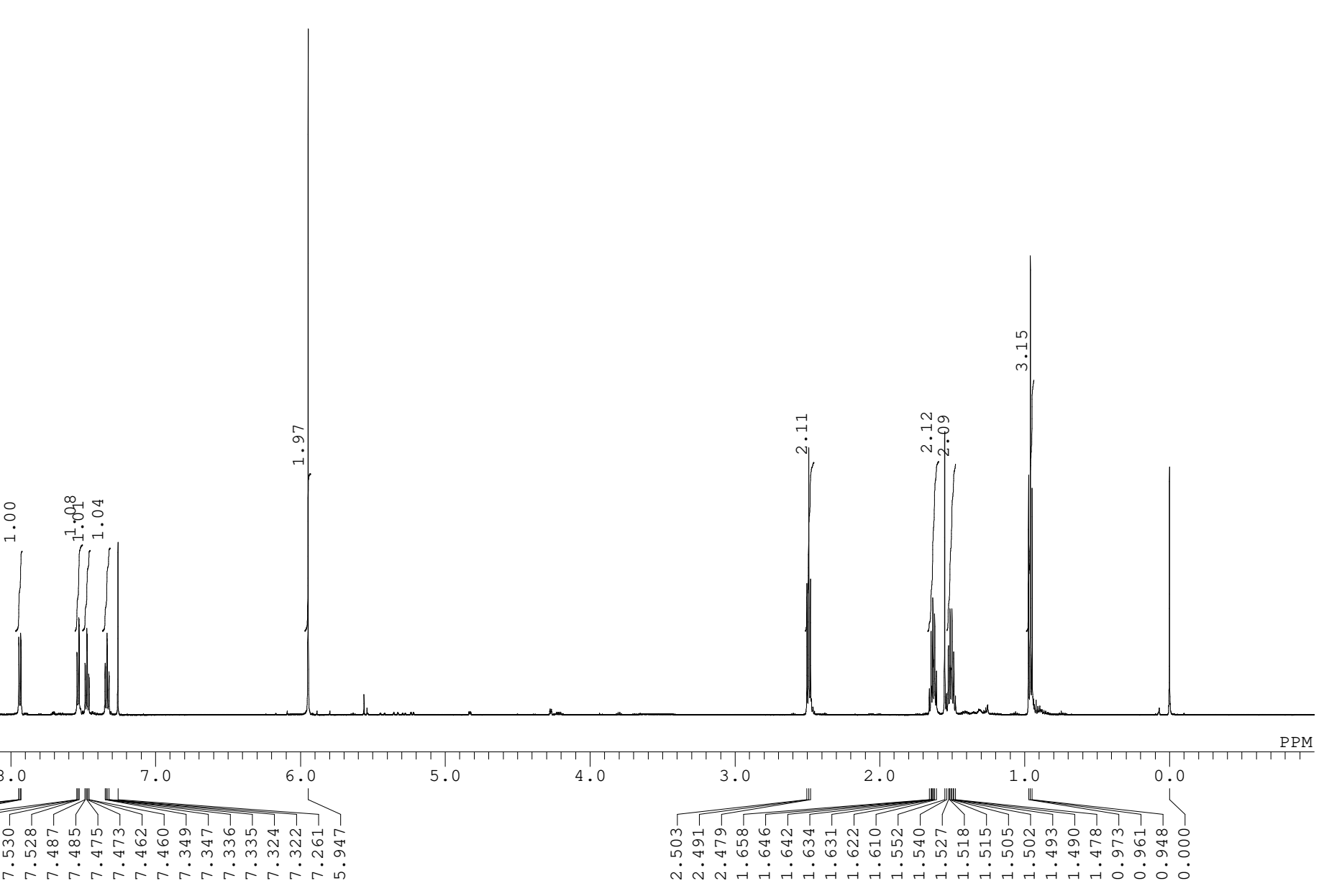




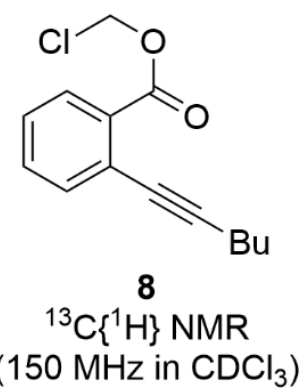

${ }^{13} \mathrm{C}\left\{{ }^{1} \mathrm{H}\right\}$ NMR spectrum of 8

(150 $\mathrm{MHz}$ in $\mathrm{CDCl}_{3}$ )

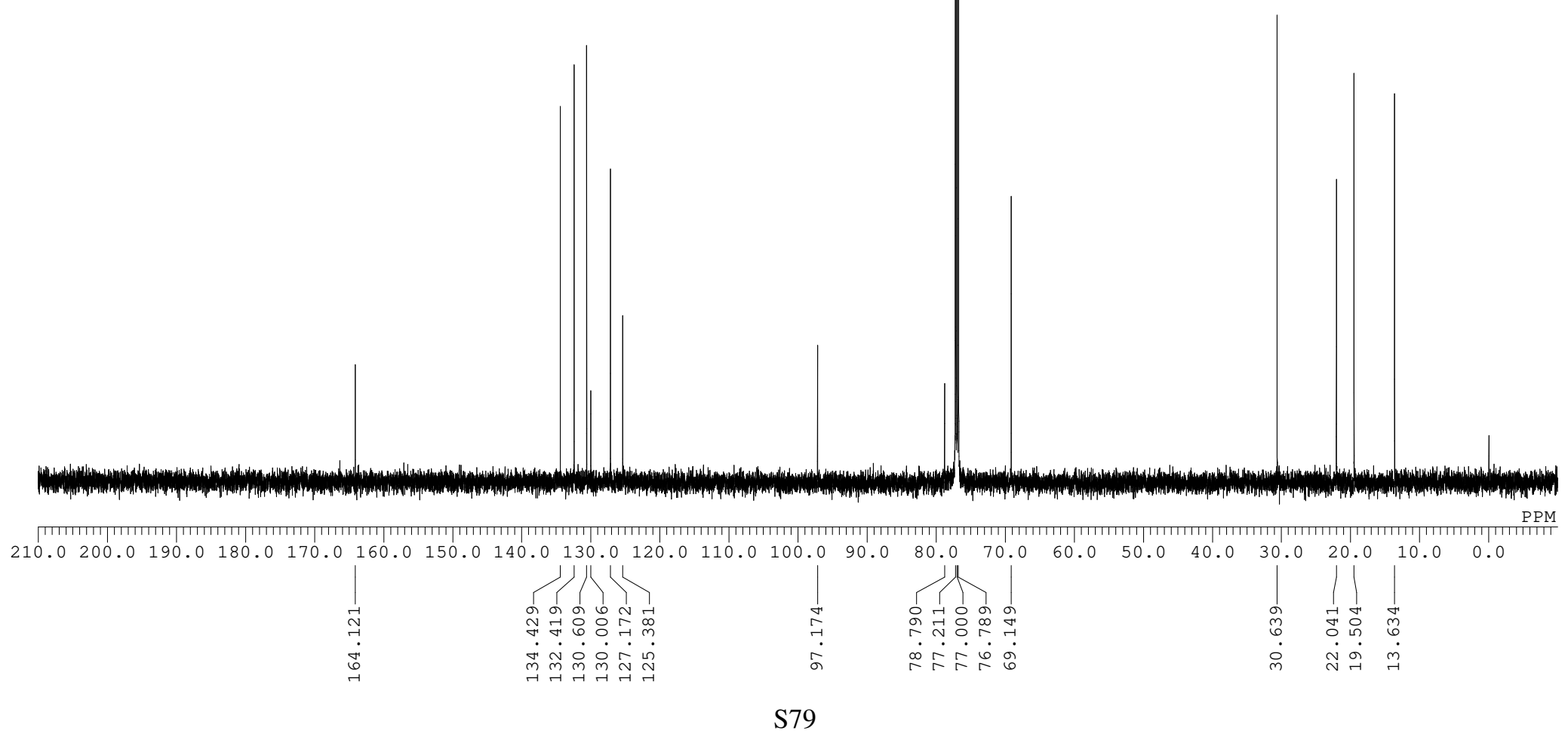




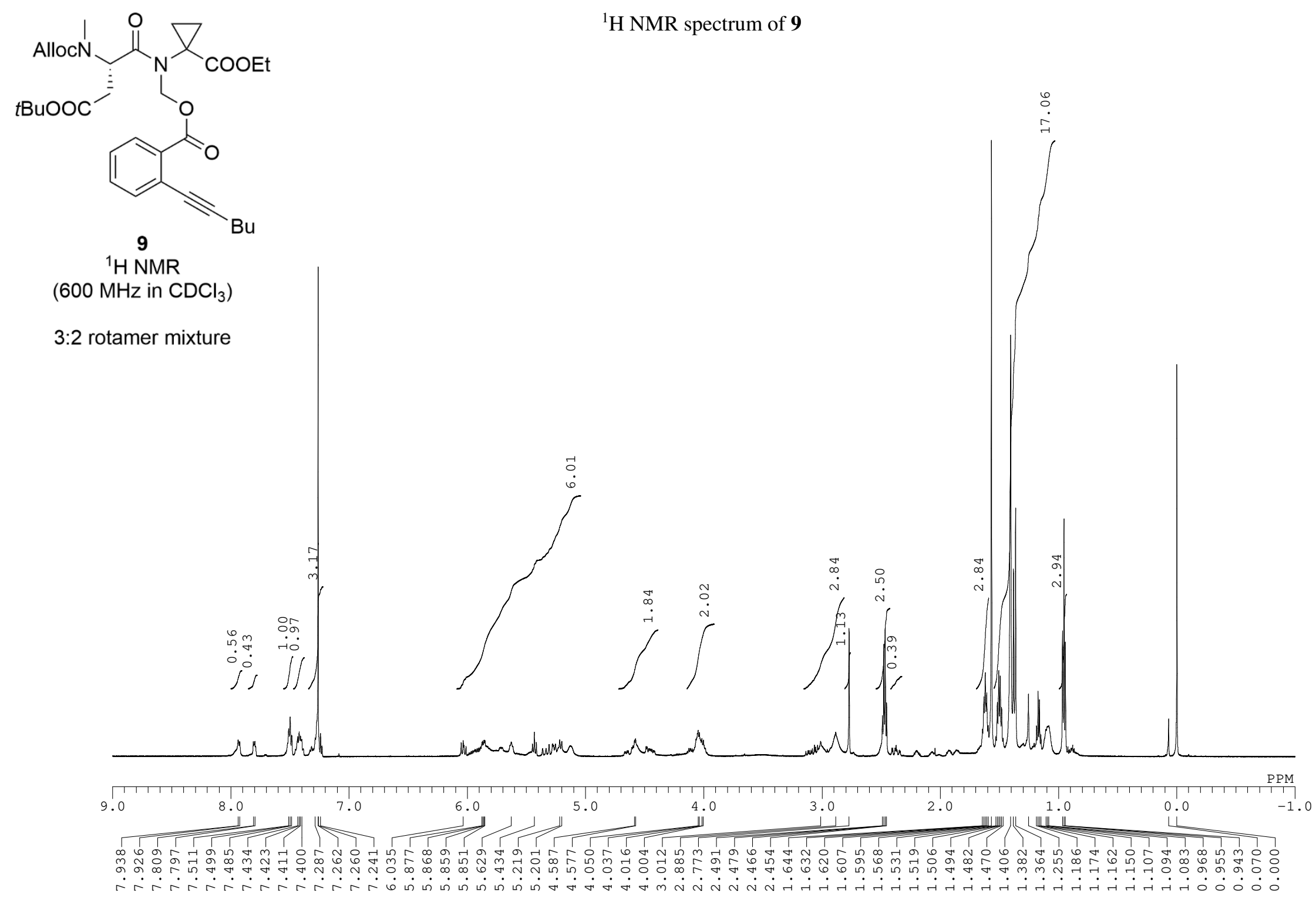




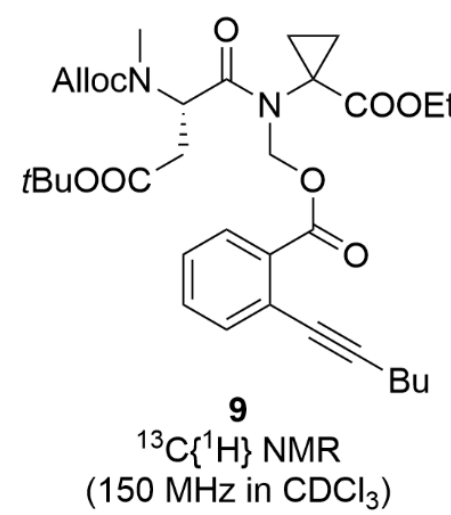

${ }^{13} \mathrm{C}\left\{{ }^{1} \mathrm{H}\right\}$ NMR spectrum of 9

$\left(150 \mathrm{MHz}\right.$ in $\left.\mathrm{CDCl}_{3}\right)$

$3: 2$ rotamer mixture

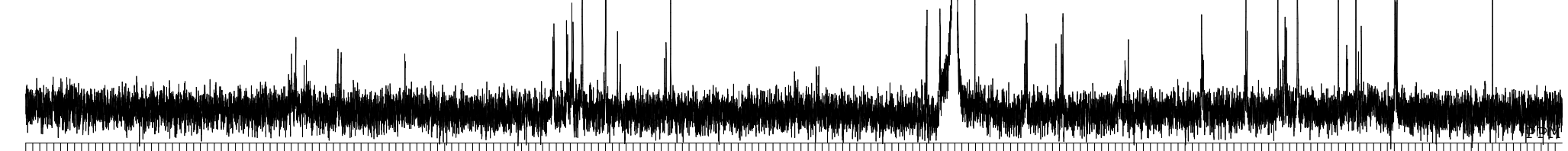

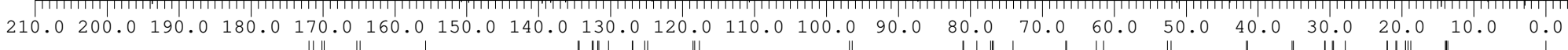

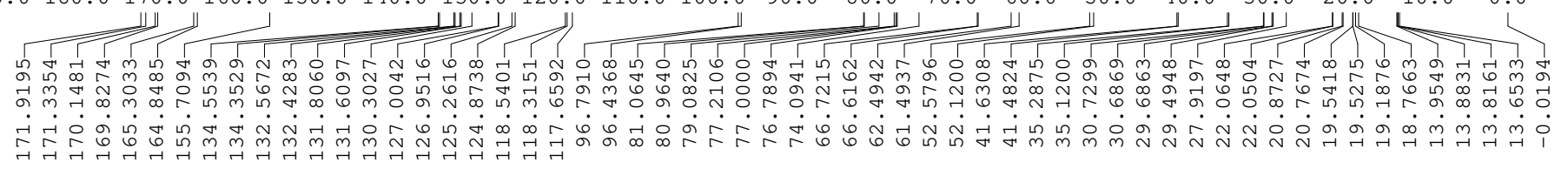




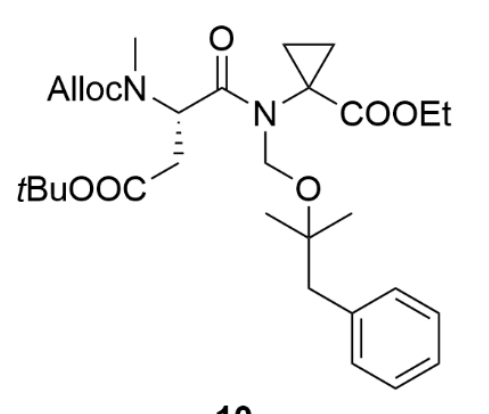

${ }^{1} \mathrm{H}$ NMR spectrum of $\mathbf{1 0}$

${ }^{1} \mathrm{H}$ NMR

$\left(600 \mathrm{MHz}\right.$ in $\mathrm{CD}_{3} \mathrm{CN}, 50^{\circ} \mathrm{C}$ )

2:1 rotamer mixture

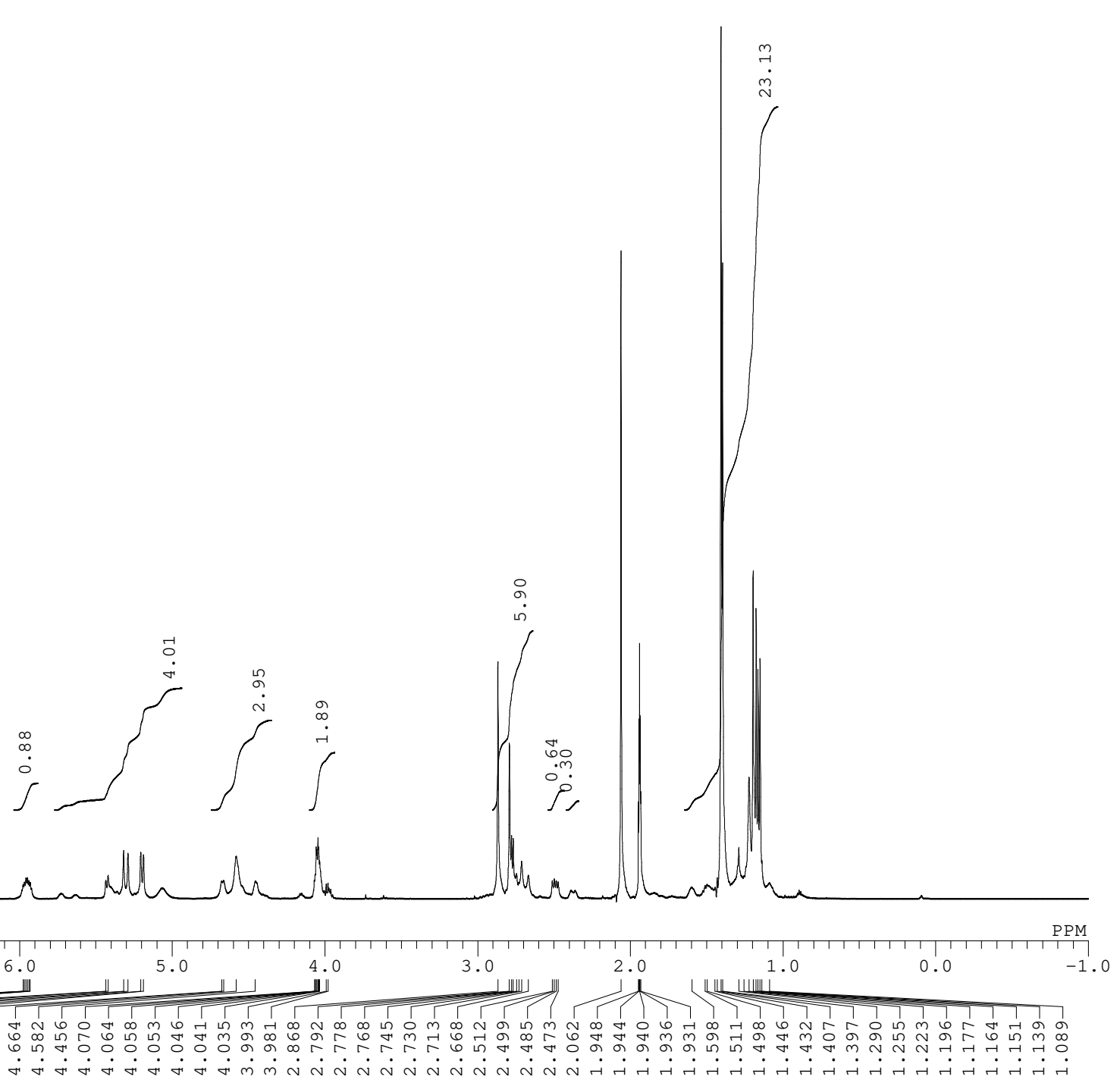




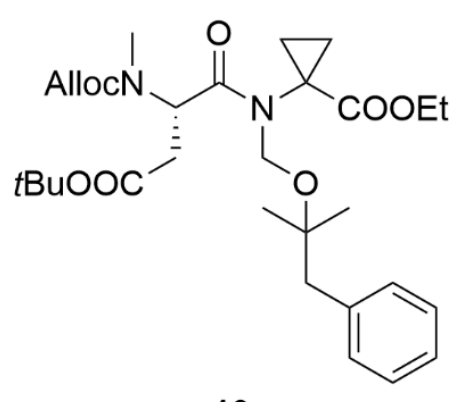

${ }^{13} \mathrm{C}\left\{{ }^{1} \mathrm{H}\right\}$ NMR spectrum of $\mathbf{1 0}$

${ }^{3} \mathrm{C}\left\{{ }^{1} \mathrm{H}\right\} \mathrm{NMR}$

$\left(150 \mathrm{MHz}\right.$ in $\left.\mathrm{CD}_{3} \mathrm{CN}, 50^{\circ} \mathrm{C}\right)$

2:1 rotamer mixture

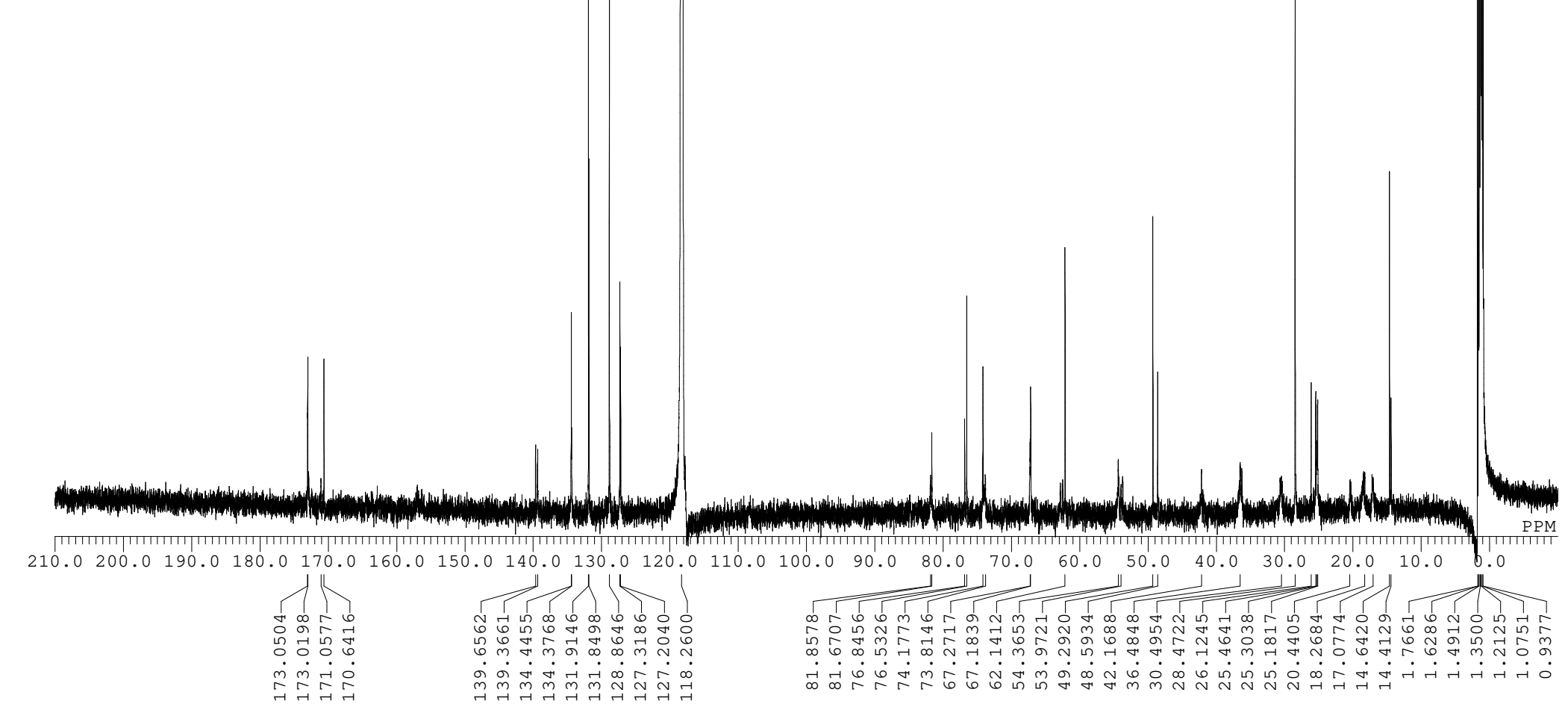

